TRANSACTIONS OF THE

AMERICAN MATHEMATICAL SOCIETY

Volume 358, Number 12, December 2006, Pages 5351-5400

S 0002-9947(06)04033-5

Article electronically published on July 20, 2006

\title{
BOUNDARY RELATIONS AND THEIR WEYL FAMILIES
}

\author{
VLADIMIR DERKACH, SEPPO HASSI, MARK MALAMUD, AND HENK DE SNOO
}

Abstract. The concepts of boundary relations and the corresponding Weyl families are introduced. Let $S$ be a closed symmetric linear operator or, more generally, a closed symmetric relation in a Hilbert space $\mathfrak{H}$, let $\mathcal{H}$ be an auxiliary Hilbert space, let

$$
J_{\mathfrak{H}}=\left(\begin{array}{cc}
0 & -i I_{\mathfrak{H}} \\
i I_{\mathfrak{H}} & 0
\end{array}\right)
$$

and let $J_{\mathcal{H}}$ be defined analogously. A unitary relation $\Gamma$ from the Kreŭn space $\left(\mathfrak{H}^{2}, J_{\mathfrak{H}}\right)$ to the Krĕn space $\left(\mathcal{H}^{2}, J_{\mathcal{H}}\right)$ is called a boundary relation for the adjoint $S^{*}$ if $\operatorname{ker} \Gamma=S$. The corresponding Weyl family $M(\lambda)$ is defined as the family of images of the defect subspaces $\widehat{\mathfrak{N}}_{\lambda}, \lambda \in \mathbb{C} \backslash \mathbb{R}$, under $\Gamma$. Here $\Gamma$ need not be surjective and is even allowed to be multi-valued. While this leads to fruitful connections between certain classes of holomorphic families of linear relations on the complex Hilbert space $\mathcal{H}$ and the class of unitary relations $\Gamma:\left(\mathfrak{H}^{2}, J_{\mathfrak{H}}\right) \rightarrow\left(\mathcal{H}^{2}, J_{\mathcal{H}}\right)$, it also generalizes the notion of so-called boundary value space and essentially extends the applicability of abstract boundary mappings in the connection of boundary value problems. Moreover, these new notions yield, for instance, the following realization theorem: every $\mathcal{H}$-valued maximal dissipative (for $\lambda \in \mathbb{C}_{+}$) holomorphic family of linear relations is the Weyl family of a boundary relation, which is unique up to unitary equivalence if certain minimality conditions are satisfied. Further connections between analytic and spectral theoretical properties of Weyl families and geometric properties of boundary relations are investigated, and some applications are given.

\section{INTRODUCTION}

Up until the seventies most papers about the extension theory of symmetric operators in a Hilbert space were mainly based on von Neumann's formula or a simplified version of it when the symmetric operator has points of regular type on the real line. Later an alternative approach was proposed by V.M. Bruck and A.N. Kochubei (see [20] and the references therein), which is based on an abstract version of Green's identity. The basic object that arises here is the notion of a boundary triplet, also called a boundary value space; see [20, 15, 16, 12,

Definition $1.1([20]$ ). Let $S$ be a closed densely defined symmetric operator in a Hilbert space $\mathfrak{H}$. A triplet $\left\{\mathcal{H}, \Gamma_{0}, \Gamma_{1}\right\}$, where $\mathcal{H}$ is a Hilbert space and $\Gamma_{i}, i=0,1$,

Received by the editors September 15, 2004.

2000 Mathematics Subject Classification. Primary 47A06, 47A20, 47A56, 47B25; Secondary 47A48, 47B50.

Key words and phrases. Symmetric operator, selfadjoint extension, Kreĭn space, unitary relation, boundary triplet, boundary relation, Weyl function, Weyl family, Nevanlinna family.

The present research was supported by the Research Institute for Technology at the University of Vaasa and by the Academy of Finland (projects 203226, 208055). 
are operators from $\operatorname{dom} S^{*}$ to $\mathcal{H}$, is said to be an (ordinary) boundary triplet for $S^{*}$, if:

(BT1) the abstract Green's identity

$$
\left(S^{*} f, g\right)-\left(f, S^{*} g\right)=\left(\Gamma_{1} f, \Gamma_{0} g\right)_{\mathcal{H}}-\left(\Gamma_{0} f, \Gamma_{1} g\right)_{\mathcal{H}}
$$

holds for all $f, g \in \operatorname{dom} S^{*}$;

(BT2) the linear mapping $\Gamma:=\left\{\Gamma_{0}, \Gamma_{1}\right\}: \operatorname{dom} S^{*} \rightarrow \mathcal{H} \oplus \mathcal{H}$ is surjective.

In [14, 15] the concept of a Weyl function was associated to an ordinary boundary triplet as an abstract version of the so-called $m$-function appearing in boundary value problems for differential operators.

Definition $1.2([14,15])$. Let $\Pi=\left\{\mathcal{H}, \Gamma_{0}, \Gamma_{1}\right\}$ be a boundary triplet for $S^{*}$. The operator-valued function $M(\lambda)$ defined by

$$
\Gamma_{1} f_{\lambda}=M(\lambda) \Gamma_{0} f_{\lambda}, \quad f_{\lambda} \in \mathfrak{N}_{\lambda}:=\operatorname{ker}\left(S^{*}-\lambda\right), \quad \lambda \in \mathbb{C} \backslash \mathbb{R},
$$

is called the Weyl function, corresponding to the boundary triplet $\Pi$.

The mappings $\Gamma_{0}$ and $\Gamma_{1}$ induce two selfadjoint extensions $A_{0}$ and $A_{1}$ of $S$, given by $\operatorname{dom} A_{i}:=\operatorname{ker} \Gamma_{i}, i=0,1$. It turns out that the Weyl function $M(\cdot)$ is an operator-valued function with values in $[\mathcal{H}]$, which is holomorphic on $\rho\left(A_{0}\right)$. Here the following notation is used: $[\mathfrak{X}, \mathfrak{Y}]$ stands for the set of all bounded linear operators between the Banach spaces $\mathfrak{X}$ and $\mathfrak{Y}$, and when $\mathfrak{Y}=\mathfrak{X}$ this is abbreviated to $[\mathfrak{X}]$. The Weyl function $M(\cdot)$ determines the pair $\left\{A_{0}, A_{1}\right\}$ up to unitary equivalence. In particular, the resolvent set of $A_{0}$ (of $A_{1}$ ) coincides with the domain of holomorphy of $M(\cdot)$ (of $\left.M^{-1}(\cdot)\right)$.

The Weyl function $M(\cdot)$ plays an important role in the spectral theory of the selfadjoint extension $A_{0}$ of $S$. It follows from (1.1) and (1.2) that the Weyl function $M(\cdot)$ satisfies the identity

$$
\frac{M(\lambda)-M(\mu)^{*}}{\lambda-\bar{\mu}}=\gamma(\mu)^{*} \gamma(\lambda), \quad \lambda, \mu \in \rho\left(A_{0}\right),
$$

where the so-called $\gamma$-field $\gamma(\lambda)$ is defined by $\gamma(\lambda)=\left(\Gamma_{0} \mid \mathfrak{N}_{\lambda}\right)^{-1}$. This implies that the Weyl function belongs to the class $R[\mathcal{H}]$ of Nevanlinna functions (also called Pick or Herglotz functions; see [18, [19, 21]) consisting of $[\mathcal{H}]$-valued functions $M(\cdot)$, holomorphic on $\mathbb{C} \backslash \mathbb{R}$, which satisfy the conditions $M(\lambda)=M(\bar{\lambda})^{*}$ and $(\operatorname{Im} \lambda)(\operatorname{Im} M(\lambda)) \geq 0, \lambda \in \mathbb{C} \backslash \mathbb{R}$. Every function $M(\cdot) \in R[\mathcal{H}]$ admits the following integral representation:

$$
M(\lambda)=A+B \lambda+\int_{\mathbb{R}}\left(\frac{1}{t-\lambda}-\frac{t}{t^{2}+1}\right) d \Sigma(t), \quad \int_{\mathbb{R}} \frac{d \Sigma(t)}{t^{2}+1} \in[\mathcal{H}],
$$

where $A=A^{*} \in[\mathcal{H}], 0 \leq B=B^{*} \in[\mathcal{H}]$, the $[\mathcal{H}]$-valued family $\Sigma(\cdot)$ is nondecreasing, and the integral is uniformly convergent in the strong topology; cf. 7], 21. In the case when the operator $S$ is densely defined the corresponding Weyl function $M(\cdot)$ satisfies two additional conditions

$$
B=\lim _{y \rightarrow \infty} \frac{M(i y)}{i y}=0 \quad \text { and } \quad \lim _{y \rightarrow \infty} y \operatorname{Im}(M(i y) h, h)=\infty, \quad 0 \neq h \in \mathcal{H} .
$$

Example 1.3. The motivation for the introduction of Weyl functions goes back to the theory of singular Sturm-Liouville operators. Let $L=-d^{2} / d t^{2}+q(t)$ be a Sturm-Liouville operator in the Hilbert space $L^{2}(0, \infty)$ with a real potential $q(\cdot)$, 
which is assumed to be in the limit-point case at $\infty$. The corresponding minimal operator $S$ is densely defined, closed, and symmetric; its defect numbers are $(1,1)$. For $y$ in the domain of the corresponding maximal operator $S^{*}$ one can define a boundary triplet $\left\{\mathbb{C}, \Gamma_{0}, \Gamma_{1}\right\}$ for $S^{*}$ by the equalities

$$
\Gamma_{0} y=y(0), \quad \Gamma_{1} y=y^{\prime}(0) .
$$

Then the corresponding Weyl function $M(\cdot)$ coincides with the $m$-function introduced originally by H. Weyl [40, and E.C. Titchmarsh [39, also called the Titchmarsh-Weyl coefficient. Let $\varphi(\cdot, \lambda)$ and $\psi(\cdot, \lambda)$ denote the fundamental solutions of the system $L u=\lambda u$ on $(0, \infty)$ which satisfy the initial conditions

$$
\begin{cases}\varphi(0, \lambda)=1, & \psi(0, \lambda)=0 \\ \varphi^{\prime}(0, \lambda)=0, & \psi^{\prime}(0, \lambda)=1 .\end{cases}
$$

The limit-point case at $\infty$ yields the fact that

$$
\gamma(t, \lambda)=\varphi(t, \lambda)+M(\lambda) \psi(t, \lambda), \quad \lambda \in \rho\left(A_{0}\right),
$$

is the only solution of $L u=\lambda u$ for $\lambda \in \mathbb{C} \backslash \mathbb{R}$ which belongs to $L^{2}(0, \infty)$ (up to constant multiples), and it satisfies

$$
\frac{M(\lambda)-\overline{M(\lambda)}}{\lambda-\bar{\lambda}}=\int_{0}^{\infty}|\gamma(t, \lambda)|^{2} d t, \quad \lambda \in \mathbb{C} \backslash \mathbb{R} .
$$

Since $\Gamma_{0} \gamma(\cdot, \lambda)=\gamma(0, \lambda)=1$ and $\Gamma_{1} \gamma(\cdot, \lambda)=\gamma^{\prime}(0, \lambda)=M(\lambda)$, the $\gamma$-field and the Weyl function corresponding to the boundary triplet $\left\{\mathbb{C}, \Gamma_{0}, \Gamma_{1}\right\}$ in (1.4) coincide with the functions $\gamma(\cdot)$ and $M(\cdot)$ in (1.5), while (1.6) expresses the identity (1.3).

The condition in Definition 1.1 that the operator $S$ is densely defined can be relaxed: it suffices to require that $S$ is a symmetric linear relation (multi-valued linear operator). However, if $S$ is nondensely defined, then the adjoint $S^{*}$ of $S$ is a linear relation and the mappings $\Gamma_{i}$ now belong to $\left[S^{*}, \mathcal{H}\right]$, where $S^{*}$ is considered as a subspace of $\mathfrak{H}^{2}:=\mathfrak{H} \times \mathfrak{H}$ equipped with the graph norm. In this case the condition (BT1) is replaced by

$$
\left(f^{\prime}, g\right)-\left(f, g^{\prime}\right)=\left(\Gamma_{1} \widehat{f}, \Gamma_{0} \widehat{g}\right)_{\mathcal{H}}-\left(\Gamma_{0} \widehat{f}, \Gamma_{1} \widehat{g}\right)_{\mathcal{H}},
$$

with $\widehat{f}:=\left\{f, f^{\prime}\right\}, \widehat{g}:=\left\{g, g^{\prime}\right\} \in S^{*}$, and the condition (BT2) requires the closed linear mapping

$$
\Gamma:=\left\{\Gamma_{0}, \Gamma_{1}\right\}: S^{*} \rightarrow \mathcal{H} \oplus \mathcal{H}
$$

to be surjective. Moreover, the Weyl function defined by

$$
\Gamma_{1} \widehat{f}_{\lambda}=M(\lambda) \Gamma_{0} \widehat{f}_{\lambda}, \quad \widehat{f}_{\lambda}:=\left\{f_{\lambda}, \lambda f_{\lambda}\right\} \in S^{*},
$$

satisfies the equality (1.3) with the definition of the $\gamma$-field $\gamma(\lambda)$ modified accordingly; cf. [16, 28]. Nondensely defined symmetric operators or relations naturally arise in various areas, such as the moment problem, Kreln's string theory, and the spectral theory of canonical systems.

Example 1.4. The spectral theory of canonical systems is concerned with systems of differential equations of the form

$$
J \frac{d x}{d t}=\lambda H(t) x, \quad J=\left(\begin{array}{cc}
0 & -I_{n} \\
I_{n} & 0
\end{array}\right), \quad t \in[0, \infty),
$$

with a locally integrable nonnegative $2 n \times 2 n$ Hamiltonian $H(t)$. If $H(t)$ is invertible for almost all $t \in(0, \infty)$, then (1.8) induces a densely defined minimal symmetric 
operator $S$ in the associated Hilbert space $L_{H}^{2}(0, \infty)$, by means of the operator $H^{-1} J \frac{d}{d t}$. However, if $H(t)$ is singular on a subset of positive Lebesgue measure of $(0, \infty)$, then (1.8) generates a closed symmetric relation $S$, whose domain is not dense in $L_{H}^{2}(0, \infty)$ and which need not even be a graph of an operator; cf. [31, 27.

Note in this connection that every scalar Nevanlinna function coincides with a Titchmarsh-Weyl coefficient of a canonical system (1.8) with a trace normed $2 \times 2$ Hamiltonian $H$ (see [6]) which can be interpreted as the Weyl function corresponding to a boundary triplet for $S^{*}$.

Define the subclass $R^{s}[\mathcal{H}]\left(R^{u}[\mathcal{H}]\right)$ of strict (uniformly strict) Nevanlinna functions in $R[\mathcal{H}]$ as the set of all functions $M(\cdot) \in R[\mathcal{H}]$ for which $0 \notin \sigma_{p}(\operatorname{Im} M(i))$ $(0 \in \rho(\operatorname{Im} M(i)))$. The identity (1.3) means that $M(\cdot)$ is a $Q$-function of the pair $\left\{S, A_{0}\right\}$ in the sense of M.G. Kreln and H. Langer (see [25, 26]), and hence it belongs to the subclass $R^{u}[\mathcal{H}]$ (whether $S$ is densely defined or not). As a $Q$-function it determines the pair $\left\{S, A_{0}\right\}$ up to unitary equivalence. It was shown in [14, 16] that for each Nevanlinna function in $R^{u}[\mathcal{H}]$ there exists a boundary triplet in the above sense for which it is the Weyl function. In [16] the concept of boundary triplet in Definition 1.1 has been extended to the case where the corresponding Weyl function belongs to the subclass $R^{s}[\mathcal{H}]$ and the inverse result for this subclass has been established.

Now the natural problem arises of whether every Nevanlinna function in the class $R[\mathcal{H}]$ can be interpreted as a Weyl function of some generalized boundary triplet. In fact, the same question can be asked for the more general notion of an arbitrary Nevanlinna family. The class of all Nevanlinna families $M(\cdot)$ in $\mathcal{H}$ is denoted by $\widetilde{R}(\mathcal{H})$; it is the set of families of linear relations $M(\lambda): \mathcal{H} \rightarrow \mathcal{H}, \lambda \in \mathbb{C} \backslash \mathbb{R}$, i.e. $M(\lambda)$ is a linear subspace of $\mathcal{H} \times \mathcal{H}$, which satisfy

(NF1) for every $\lambda \in \mathbb{C}_{+}\left(\mathbb{C}_{-}\right)$the relation $M(\lambda)$ is maximal dissipative (resp. accumulative);

(NF2) $M(\lambda)^{*}=M(\bar{\lambda}), \lambda \in \mathbb{C} \backslash \mathbb{R}$

(NF3) for some, and hence for all, $\mu \in \mathbb{C}_{+}\left(\mathbb{C}_{-}\right)$the operator family $(M(\lambda)+\mu)^{-1}$ $(\in[\mathcal{H}])$ is holomorphic for all $\lambda \in \mathbb{C}_{+}\left(\mathbb{C}_{-}\right)$.

The class $\widetilde{R}(\mathcal{H})$ contains the class $R(\mathcal{H})$ of all Nevanlinna functions whose values are in general unbounded operators. The theory of maximal dissipative operators goes back at least to R.S. Phillips; cf. [32, 33. Later followed extensions to maximal dissipative relations and $\lambda$-depending holomorphic families (in $\mathbb{C}^{+}$) of maximal dissipative relations or, equivalently, of Nevanlinna pairs; cf. e.g. 24, 17, 26, 3. The following example illustrates the occurrence of Nevanlinna functions whose values are unbounded operators and the relevance of the above question.

Example 1.5. Let $L=-d^{2} / d t^{2}+Q$ be a Sturm-Liouville operator in the Hilbert space $L^{2}(0, \infty ; \mathcal{H})$ with a (constant) potential $Q(\geq I)$ being a bounded operator in the Hilbert space $\mathcal{H}$. Let $S$ be the minimal operator associated with $L$. Then the boundary triplet is still given by (1.4) and the corresponding Weyl function is given by

$$
M(\lambda)=\sqrt{Q-\lambda}, \quad \lambda \in \mathbb{C} \backslash[1, \infty) .
$$

In the case when $Q$ is unbounded, which occurs, for example, when $L$ is the Laplace operator in a half-plane or in a strip, the formulas (1.4) do not define a boundary 
triplet for $S^{*}$ anymore, since the boundary values $y(0)$ and $y^{\prime}(0)$ do not belong to the space $\mathcal{H}$ for $y \in \operatorname{dom} S^{*}$. Therefore the construction of a boundary triplet for $S^{*}$ in this case is nontrivial. For this purpose a regularization procedure of the boundary mappings has been developed in [20 resulting in a boundary triplet for $S^{*}$. However, this regularization procedure produces a Weyl function which is a renormalization of the function $M(\cdot)$ in (1.9) (see [15]), and this makes it difficult to investigate the appropriate spectral properties determined by the given boundary conditions.

The concept of a boundary relation introduced in the present paper does not require surjectivity of the mappings $\Gamma_{0}$ or $\Gamma_{1}$, nor do these mappings have to be defined everywhere on $S^{*}$. This makes it possible to introduce a boundary relation for $S^{*}$ in Example 1.5 by defining the mappings $\Gamma_{0}$ and $\Gamma_{1}$ in (1.4) only on "smooth vectors" from dom $S^{*}$, without any regularization procedure. The corresponding Weyl function $M(\cdot)$ is still of the form (1.9), but now it is an operator-valued $R$ function with unbounded values. The construction of a boundary relation for the operator $S^{*}$ in the new sense is much simpler than the construction of a boundary triplet for $S^{*}$. Furthermore, the corresponding generalized Weyl function $M(\lambda)=$ $\sqrt{Q-\lambda}$ makes it possible to describe the spectrum of the operator $A_{0}$ corresponding to the Dirichlet boundary value problem for the operator $S^{*}$. Namely, starting with $M(\lambda)=\sqrt{Q-\lambda}$ it can be shown that for every $Q(\geq I)$ the extension $A_{0}$ has a purely absolutely continuous spectrum.

The above problem concerning the interpretation of Nevanlinna families is also intimately related to the KreIn-Naimark formula. If $S$ is a closed symmetric relation in a Hilbert space $\mathfrak{H}$ with equal defect numbers, then the Kreı̆n-Naimark formula

$$
P_{\mathfrak{H}}(\widetilde{A}-\lambda)^{-1} \uparrow_{\mathfrak{H}}=\left(A_{0}-\lambda\right)^{-1}-\gamma(\lambda)(M(\lambda)+\tau(\lambda))^{-1} \gamma(\bar{\lambda})^{*},
$$

with $\lambda \in \mathbb{C} \backslash \mathbb{R}$, expresses a one-to-one correspondence between all selfadjoint exit space extensions $\widetilde{A}$ in a Hilbert space $\widetilde{\mathfrak{H}} \supset \mathfrak{H}$ of $S$ and the class of all Nevanlinna families $\tau(\cdot)$ in the Hilbert space $\mathcal{H}$. Here $P_{\mathfrak{H}}$ is the orthogonal projection from the exit space $\widetilde{\mathfrak{H}}$ onto $\mathfrak{H}, M(\cdot)$ is the Weyl function in $\mathcal{H}$ and $\gamma(\cdot)$ is the $\gamma$-field mapping $\mathcal{H}$ into $\mathfrak{N}_{\lambda}\left(S^{*}\right)$, corresponding to the selfadjoint extension $A_{0}$ of $S$. The new concepts of a boundary relation and the corresponding Weyl family introduced in this paper make it possible to interpret the Nevanlinna family $\tau(\cdot)$ in the resolvent formula (1.10) as a Weyl family for the symmetric relation $S_{2}:=\widetilde{A} \cap(\widetilde{\mathfrak{H}} \ominus \mathfrak{H})^{2}$. Moreover, following the ideas in [12], the exit space extension $\widetilde{A}$ can be constructed as a coupling of the relations $S$ and $S_{2}$. This leads to a general and useful "linearization method" to investigate, for instance, spectral problems involving spectral parameters in the boundary conditions. In the special case when $\tau(\cdot) \in R^{u}[\mathcal{H}]$ these constructions have been established by the authors in [12]; see also [11. The general case will be carried out in a forthcoming paper. Recently the coupling method for the construction of generalized resolvents in 12 has been applied by J. Behrndt and P. Jonas 4. Their treatment involves a scalar function $\tau(\cdot)$, belonging to the class of local generalized Nevanlinna functions, which generates a selfadjoint exit space extension in a Krel̆n space. The next example illustrates the above ideas and the occurrence of Nevanlinna families, which are not necessarily (graphs of) operators, in connection with boundary value problems.

Example 1.6. Consider the following boundary value problem for the SturmLiouville operator $L=-d^{2} / d t^{2}+q(t)$ in Example 1.3, but now in $\mathfrak{H}=L^{2}(0, \infty ; \mathcal{H})$ 
with $\mathcal{H}=\mathbb{C}^{n}$, assuming that $q(\cdot)$ is a selfadjoint $n \times n$ matrix potential such that the limit point case prevails at $\infty$ :

$$
L y=\lambda y, \quad \Phi(\lambda) y^{\prime}(0)+\Psi(\lambda) y(0)=0 .
$$

Here $\{\Phi(\cdot), \Psi(\cdot)\}$ is a Nevanlinna pair, which means that

$$
\tau(\lambda)=\{\{\Phi(\lambda) h, \Psi(\lambda) h\}: h \in \mathcal{H}\}
$$

is a Nevanlinna family, which is not operator-valued when $\Phi(\lambda)$ is not invertible. Denote by $S$ the corresponding minimal symmetric operator which, by assumption, has defect numbers $(n, n)$. Let $M(\cdot)$ be the Weyl function of $S$ corresponding to the boundary triplet (1.4). The solution of the equation $L y-\lambda y=g$, subject to the boundary conditions (1.11), admits a representation

$$
y(\cdot, \lambda)=P_{\mathfrak{H}}(\widetilde{A}-\lambda)^{-1} g, \quad g \in \mathfrak{H},
$$

where $P_{\mathfrak{H}}(\widetilde{A}-\lambda)^{-1} \Gamma_{\mathfrak{H}}$ is a generalized resolvent of $S$ as in (1.10). Following [12] and applying the main realization result of the present paper, the extension $\widetilde{A}$ (i.e., a linearization of the spectral problem (1.11) ) can be constructed as a coupling of the minimal Sturm-Liouville operator $S$ and a symmetric relation $S_{2}$ with the Weyl family $\tau(\cdot)$, independent of the invertibility of $\Phi(\cdot)$. It is in this way that multi-valued families $\tau(\cdot)$ naturally arise in the connection of boundary value problems. Using the coupling conditions for $S$ and $S_{2}$ the calculation of the compressed resolvent in (1.12) gives the Kreĭn-Naimark resolvent formula (1.10); cf. [11], [12].

In order to explain the new notions assume for the moment that $S$ is densely defined and rewrite Green's identity (1.1) in the assumption (BT1) of Definition 1.1 as

$$
\left(S^{*} f, g\right)-\left(\Gamma_{1} f, \Gamma_{0} g\right)_{\mathcal{H}}=\left(f, S^{*} g\right)-\left(\Gamma_{0} f, \Gamma_{1} g\right)_{\mathcal{H}}, \quad f, g \in \operatorname{dom} S^{*} .
$$

The interpretation of (1.13) is that the operator $\widetilde{A}$ defined by

$$
\widetilde{A}:\left(\begin{array}{c}
f \\
\Gamma_{0} f
\end{array}\right) \mapsto\left(\begin{array}{c}
S^{*} f \\
-\Gamma_{1} f
\end{array}\right), \quad f \in \operatorname{dom} S^{*},
$$

is symmetric in $\mathfrak{H} \times \mathcal{H}$. Moreover, the assumption (BT2) of Definition1.1 guarantees that $\widetilde{A}$ is selfadjoint in $\mathfrak{H} \times \mathcal{H}$. If $S$ is not densely defined, similar observations can be made when (1.14) is appropriately interpreted. The precise definition of a boundary relation will be given in Section 3 , but in an equivalent form it can be reformulated as follows. A pair $\{\mathcal{H}, \Gamma\}$, where $\Gamma: \mathfrak{H}^{2} \rightarrow \mathcal{H}^{2}$ is a closed linear relation (i.e. a linear subspace of $\mathfrak{H}^{2} \times \mathcal{H}^{2}$ ), is said to be a boundary relation for $S^{*}$ if $\operatorname{dom} \Gamma$ is dense in $S^{*}$ and if the transform $\widetilde{A}=\mathcal{J}(\Gamma)$ of $\Gamma$ determined by

$$
\widetilde{A}=\mathcal{J}(\Gamma):=\left\{\left\{\left(\begin{array}{l}
f \\
h
\end{array}\right),\left(\begin{array}{c}
f^{\prime} \\
-h^{\prime}
\end{array}\right)\right\}:\left\{\left(\begin{array}{c}
f \\
f^{\prime}
\end{array}\right),\left(\begin{array}{c}
h \\
h^{\prime}
\end{array}\right)\right\} \in \Gamma\right\}
$$

is a selfadjoint relation in $\mathfrak{H} \times \mathcal{H}$. The linear relation $\Gamma$ from the Kreй space $\left(\mathfrak{H}^{2}, J_{\mathfrak{H}}\right)$ to the Krein space $\left(\mathcal{H}^{2}, J_{\mathcal{H}}\right)$ turns out to be unitary in the sense of relations; cf. 34. In this definition $S$ is not necessarily densely defined, and $S$ is allowed to have infinite and unequal defect numbers. The corresponding Weyl family is now defined by

$$
M(\lambda)=\Gamma\left(\left\{\left\{f, f^{\prime}\right\} \in \operatorname{dom} \Gamma: f^{\prime}=\lambda f\right\}\right)
$$


as an extension of Definition 1.2. The given assumptions are enough to guarantee that the Weyl family $M(\cdot)$ is a Nevanlinna family in the sense of Definition (NF1)(NF3).

The main result in this paper shows that every Nevanlinna family can be realized as a Weyl family of some boundary relation which is unique, up to unitary equivalence, when a certain minimality condition is satisfied; see Theorem 3.9. This establishes a new general technique for investigating problems and various applications where Nevanlinna families or their various special cases appear. The proof of Theorem 3.9 is based on the generalized Naimark theorem and does not use any operator model as was done in the case of a uniformly strict Nevanlinna function (see [25], 26], 16]). Note in this connection that a simple proof of the Naimark dilation theorem was recently presented in [29]. Observe that the definition of boundary relation allows $\Gamma$ to be multi-valued in which case it may happen that $\Gamma$ is indecomposable into the orthogonal sum $\Gamma_{0} \oplus \Gamma_{1}$, where $\Gamma_{j}: \mathfrak{H}^{2} \rightarrow \mathcal{H}$, $j=0,1$ (see (3.6)). When such a decomposition makes sense, the new concept of the boundary relation reduces to a natural generalization of the notion of an ordinary boundary triplet in Definition 1.1 as well as of the notion of a generalized boundary triplet in [16]; in this case the notation "boundary triplet" will still be kept for $\Gamma$ in the present paper.

The connection (1.15) between the boundary relation $\Gamma$ and the selfadjoint operator or, in general, relation $\widetilde{A}$ plays a fundamental role in the sequel. The interpretation of $\widetilde{A}$ is that of a selfadjoint exit space extension of $S$ determined by the boundary relation $\Gamma$. The given procedure can be applied, for instance, in the linearization of boundary value problems with eigenvalue parameters in the boundary conditions; here arbitrary (finite or infinite, and equal or unequal) defect numbers for the underlying operators are allowed. The appearance of unbounded Weyl functions is not excluded here either; this makes it unnecessary to find regularizations for boundary mappings for treating boundary value problems involving partial differential operators; cf. [20, [16]. Some recent physical applications can be found, for instance, in [8], [9]. In forthcoming papers these matters will be further investigated, including the extension of the notions of boundary relations and the corresponding Weyl families to the case where $S$ is defined on a space with an indefinite inner product. The present paper establishes for the first time on a general level the link between the abstract boundary triplets (here the mapping $\Gamma$ ) and the exit space extensions $\widetilde{A}$ (via the transform $\mathcal{J}$ which connects $\Gamma$ and $\widetilde{A}$ ). In what follows, this connection is effectively used in building up the general theory of boundary relations and their Weyl families, and it plays a key role in proving some of the central theorems of the present paper. Some of the main results of the paper have been announced in [13.

In Section 2 some preparatory material is presented, including results on linear relations in Kreln spaces. Here the main transform $\mathcal{J}$ acting between two Krel̆n spaces is introduced, and its properties are investigated. In Section 3 the concepts of a boundary relation and the corresponding Weyl family are introduced. The main result of this section is the following inverse theorem: every Nevanlinna family can be realized as the Weyl function of a boundary relation. In Section 4 the investigation of geometrical properties of boundary relations and the analytical properties of the corresponding Weyl families is continued. Several known results on $Q$-functions or, equivalently, Weyl functions of ordinary boundary triplets are extended to wider 
subclasses of Nevanlinna families. In particular, geometrical properties of boundary relations whose Weyl families $M(\lambda)$ are domain invariant are studied in detail. In Section 5 the connection between the new concepts and the earlier concepts of boundary triplets and the corresponding Weyl functions is investigated. Section 6 contains several examples which demonstrate the applicability of the new concepts and sharpness of several statements in the earlier sections of the paper, as well as some new unexpected effects.

\section{Preliminaries}

2.1. Linear relations in linear spaces. This paper is concerned with (graphs of) linear operators and linear relations in linear spaces. The following notation will be used systematically. The Cartesian product $\mathfrak{H} \times \mathfrak{H}^{\prime}$ of linear spaces $\mathfrak{H}$ and $\mathfrak{H}^{\prime}$ is the set of all ordered pairs (of $1 \times 2$ matrices) $\left\{f, f^{\prime}\right\}$ with $f \in \mathfrak{H}$ and $f^{\prime} \in \mathfrak{H}^{\prime}$. Frequently it will be convenient to denote the Cartesian product $\mathfrak{H} \times \mathfrak{H}^{\prime}$ and the elements of it (as $2 \times 1$ matrices) by

$$
\left(\begin{array}{c}
f \\
f^{\prime}
\end{array}\right) \in\left(\begin{array}{c}
\mathfrak{H} \\
\mathfrak{H}^{\prime}
\end{array}\right), \quad f \in \mathfrak{H}, \quad f^{\prime} \in \mathfrak{H}^{\prime} .
$$

If $\mathfrak{L} \subset \mathfrak{H}$ and $\mathfrak{L}^{\prime} \subset \mathfrak{H}^{\prime}$ are linear subspaces, then $\mathfrak{L} \times \mathfrak{L}^{\prime}$ denotes the Cartesian product of the subspaces; in agreement with the ordered pairs this product will also be denoted by $\left\{\mathfrak{L}, \mathfrak{L}^{\prime}\right\}$, or by $\mathfrak{L}^{2}$ if $\mathfrak{L}=\mathfrak{L}^{\prime}$.

A linear relation $T$ from $\mathfrak{H}$ to $\mathfrak{H}^{\prime}$ is a linear subspace of $\mathfrak{H} \times \mathfrak{H}^{\prime}$. Systematically a linear operator $T$ will be identified with its graph. It is convenient to write $T: \mathfrak{H} \rightarrow \mathfrak{H}^{\prime}$ and interpret the linear relation $T$ as a multi-valued linear mapping from $\mathfrak{H}$ into $\mathfrak{H}^{\prime}$. If $\mathfrak{H}^{\prime}=\mathfrak{H}$ one speaks of a linear relation $T$ in $\mathfrak{H}$.

If $T$ is a linear relation from $\mathfrak{H}^{2}=\mathfrak{H} \times \mathfrak{H}$ to $\mathfrak{H}^{\prime 2}=\mathfrak{H}^{\prime} \times \mathfrak{H}^{\prime}$, then an element of $T$ will be denoted by $\{\widehat{f}, \widehat{h}\}$ with the understanding that $\widehat{f}=\left\{f, f^{\prime}\right\} \in \mathfrak{H}^{2}$ and $\widehat{h}=\left\{h, h^{\prime}\right\} \in \mathfrak{H}^{\prime 2}$. It will also be convenient to think of such a general element as

$$
\{\widehat{f}, \widehat{h}\}=\left\{\left(\begin{array}{c}
f \\
f^{\prime}
\end{array}\right),\left(\begin{array}{c}
h \\
h^{\prime}
\end{array}\right)\right\} \quad \text { with } \quad \widehat{f}=\left(\begin{array}{c}
f \\
f^{\prime}
\end{array}\right) \in\left(\begin{array}{c}
\mathfrak{H} \\
\mathfrak{H}
\end{array}\right), \quad \widehat{h}=\left(\begin{array}{c}
h \\
h^{\prime}
\end{array}\right) \in\left(\begin{array}{c}
\mathfrak{H}^{\prime} \\
\mathfrak{H}^{\prime}
\end{array}\right),
$$

according to the interpretation explained above. This interpretation will be assumed whenever needed without explicit mention.

For a linear relation $T: \mathfrak{H} \rightarrow \mathfrak{H}^{\prime}$ the symbols $\operatorname{dom} T, \operatorname{ker} T, \operatorname{ran} T$, and mul $T$ stand for the domain, kernel, range, and the multi-valued part, respectively. The inverse $T^{-1}$ is a relation from $\mathfrak{H}^{\prime}$ to $\mathfrak{H}$ defined by $\left\{\left\{f^{\prime}, f\right\}:\left\{f, f^{\prime}\right\} \in T\right\}$. The sum $T_{1}+T_{2}$ and the componentwise sum $T_{1} \widehat{+} T_{2}$ of two linear relations $T_{1}$ and $T_{2}$ are defined by

$$
\begin{aligned}
& T_{1}+T_{2}=\left\{\{f, g+h\}:\{f, g\} \in T_{1},\{f, h\} \in T_{2}\right\}, \\
& T_{1} \widehat{+} T_{2}=\left\{\{f+h, g+k\}:\{f, g\} \in T_{1},\{h, k\} \in T_{2}\right\} .
\end{aligned}
$$

If the componentwise sum is orthogonal it will be denoted by $T_{1} \oplus T_{2}$. Clearly,

$$
\operatorname{ker}\left(T_{1}-T_{2}\right)=\operatorname{dom}\left(T_{1} \cap T_{2}\right), \quad \operatorname{ker}\left(T_{1}^{-1}-T_{2}^{-1}\right)=\operatorname{ran}\left(T_{1} \cap T_{2}\right) .
$$

The null spaces of $T-\lambda, \lambda \in \mathbb{C}$, are defined by

$$
\mathfrak{N}_{\lambda}(T)=\operatorname{ker}(T-\lambda), \quad \widehat{\mathfrak{N}}_{\lambda}(T)=\left\{\{f, \lambda f\} \in T: f \in \mathfrak{N}_{\lambda}(T)\right\} .
$$

Moreover, $\rho(T)(\hat{\rho}(T))$ stands for the set of regular (regular type) points of $T$. The closure of a linear relation $T$ will be denoted by $\operatorname{clos} T$. Note that $\operatorname{dom} T \subset$ 
$\operatorname{dom}(\operatorname{clos} T)$ and $\operatorname{ran} T \subset \operatorname{ran}(\operatorname{clos} T)$, with equality when $\operatorname{dom} T$ or $\operatorname{ran} T$ are closed, respectively.

Recall the following simple result (cf. [16, Lemma 2.1]) which will be used in the analysis of linear relations.

Lemma 2.1 ([16). Let $\mathfrak{X}$ and $\mathfrak{Y}$ be Banach spaces, let $\mathfrak{M}$ be a closed linear subspace of $\mathfrak{X}$, and let $P \in[\mathfrak{X}, \mathfrak{Y}]$ be surjective. Then the range $P \mathfrak{M}$ is closed in $\mathfrak{Y}$ if and only if the sum of the linear subspaces $\mathfrak{M}+\mathfrak{N}$ is closed in $\mathfrak{X}$, where $\mathfrak{N}:=\operatorname{ker} P$.

2.2. Linear relations in Hilbert spaces. Let $\mathfrak{H}_{1}$ and $\mathfrak{H}_{2}$ be separable Hilbert spaces and provide the Cartesian product $\mathfrak{H}_{1} \times \mathfrak{H}_{2}$ with the usual inner product so that $\mathfrak{H}_{1} \times \mathfrak{H}_{2}$ is a Hilbert space denoted by $\mathfrak{H}_{1} \oplus \mathfrak{H}_{2}$. Let $T: \mathfrak{H}_{1} \rightarrow \mathfrak{H}_{2}$ be a linear relation from $\mathfrak{H}_{1}$ to $\mathfrak{H}_{2}$. The adjoint $T^{*}$ is the closed linear relation from $\mathfrak{H}_{2}$ to $\mathfrak{H}_{1}$ defined by (see [5], [10])

$$
T^{*}=\left\{\{h, k\} \in \mathfrak{H}_{2} \oplus \mathfrak{H}_{1}:(k, f)_{\mathfrak{H}_{1}}=(h, g)_{\mathfrak{H}_{2}},\{f, g\} \in T\right\} .
$$

Proposition 2.2. Let $T$ be a closed linear relation from a Hilbert space $\mathfrak{H}_{1}$ to a Hilbert space $\mathfrak{H}_{2}$. Then:

(i) $\operatorname{dom} T$ is closed if and only if $\operatorname{dom} T^{*}$ is closed;

(ii) $\operatorname{ran} T$ is closed if and only if $\operatorname{ran} T^{*}$ is closed.

Proof. The statement (i) is equivalent to the statement (ii) (by inversion of $T$ ). So it suffices to prove the last statement. Let $P$ be the orthoprojection from $\mathfrak{H}_{1} \oplus \mathfrak{H}_{2}$ onto $\mathfrak{H}_{2}$, so that ker $P=\mathfrak{H}_{1} \times\{0\}$. Assume that $\operatorname{ran} T=P T$ is closed. Then by Lemma $2.1 T \widehat{+}\left(\mathfrak{H}_{1} \times\{0\}\right)$ is also closed. By a theorem of Kato 22, Chapter 4, Theorem 4.8] the corresponding sum of the orthogonal complements in $\mathfrak{H}_{1} \oplus \mathfrak{H}_{2}$,

$$
T^{\perp} \widehat{+}\left(\{0\} \times \mathfrak{H}_{2}\right),
$$

is also closed. The operator $J: \mathfrak{H}_{1} \oplus \mathfrak{H}_{2} \rightarrow \mathfrak{H}_{2} \oplus \mathfrak{H}_{1}$ given by $J\left\{h, h^{\prime}\right\}=\left\{-h^{\prime}, h\right\}$ is unitary, and it follows from (2.3) that $T^{*}=J T^{\perp}$. Hence, (2.4) implies that

$$
T^{*} \widehat{+}\left(\mathfrak{H}_{2} \times\{0\}\right)
$$

is also closed. In other words, $T^{*} \widehat{+} \operatorname{ker} Q$ is closed. Here $Q$ is the orthoprojection from $\mathfrak{H}_{2} \oplus \mathfrak{H}_{1}$ onto $\mathfrak{H}_{1}$, so that $\operatorname{ker} Q=\mathfrak{H}_{2} \times\{0\}$. Another application of Lemma 2.1 shows that $Q T^{*}=\operatorname{ran} T^{*}$ is closed.

Recall that a linear relation $T$ in $\mathfrak{H}$ is called symmetric (dissipative or accumulative) if $\operatorname{Im}\left(h^{\prime}, h\right)=0$ ( $\geq 0$ or $\leq 0$, respectively) for all $\left\{h, h^{\prime}\right\} \in T$. These properties remain invariant under closures. By polarization it follows that a linear relation $T$ in $\mathfrak{H}$ is symmetric if and only if $T \subset T^{*}$. A linear relation $T$ in $\mathfrak{H}$ is called selfadjoint if $T=T^{*}$, and it is called essentially selfadjoint if $\operatorname{clos} T=T^{*}$. A dissipative (accumulative) linear relation $T$ in $\mathfrak{H}$ is called maximal dissipative (maximal accumulative) if it has no proper dissipative (accumulative) extensions. Clearly, a linear relation $T$ is selfadjoint if and only if it is both maximal dissipative and maximal accumulative.

Assume that $T$ is closed. If $T$ is dissipative or accumulative, then $\operatorname{mul} T \subset$ mul $T^{*}$. In this case the orthogonal decomposition $\mathfrak{H}=(\operatorname{mul} T)^{\perp} \oplus \operatorname{mul} T$ induces an orthogonal decomposition of $T$ as

$$
T=T_{s} \oplus T_{\infty}, \quad T_{\infty}=\{0\} \times \operatorname{mul} T, \quad T_{s}=\{\{f, g\} \in T: g \perp \operatorname{mul} T\},
$$

where $T_{\infty}$ is a selfadjoint relation in mul $T$ and $T_{s}$ is an operator in $\mathfrak{H} \ominus$ mul $T$ with $\overline{\operatorname{dom}} T_{s}=\overline{\operatorname{dom}} T=\left(\operatorname{mul} T^{*}\right)^{\perp}$, which is dissipative or accumulative. Moreover, 
if the relation $T$ is maximal dissipative or accumulative, then $\operatorname{mul} T=\operatorname{mul} T^{*}$. In this case the orthogonal decomposition $(\operatorname{dom} T)^{\perp}=\operatorname{mul} T^{*}$ shows that $T_{s}$ is a densely defined dissipative or accumulative operator in $(\operatorname{mul} T)^{\perp}$, which is maximal (as an operator). In particular, if $T$ is a selfadjoint relation, then there is such a decomposition where $T_{s}$ is a selfadjoint operator (densely defined in $\left.(\operatorname{mul} T)^{\perp}\right)$.

Let $S$ be a closed symmetric linear relation in a Hilbert spaces $\mathfrak{H}$. Then the adjoint relation $S^{*}$ can be decomposed via the von Neumann formula:

$$
S^{*}=S \widehat{+} \widehat{\mathfrak{N}}_{\lambda}\left(S^{*}\right) \widehat{+} \widehat{\mathfrak{N}}_{\bar{\lambda}}\left(S^{*}\right), \quad \lambda \in \mathbb{C} \backslash \mathbb{R}, \quad \text { direct sums },
$$

where $\widehat{\mathfrak{N}}_{\lambda}\left(S^{*}\right)$ is defined as in (2.2). When $\lambda= \pm i$ the decomposition (2.5) is orthogonal:

$$
S^{*}=S \oplus \widehat{\mathfrak{N}}_{i}\left(S^{*}\right) \oplus \widehat{\mathfrak{N}}_{-i}\left(S^{*}\right),
$$

where the orthogonality is with respect to the inner product topology in $S^{*}$; cf. [5], [10. A symmetric linear relation $S$ is called simple if there is no nontrivial orthogonal decomposition of the Hilbert space $\mathfrak{H}=\mathfrak{H}_{1} \oplus \mathfrak{H}_{2}$ and no corresponding orthogonal decomposition $S=S_{1} \oplus S_{2}$ with $S_{1}$ a symmetric relation in $\mathfrak{H}_{1}$ and $S_{2}$ a selfadjoint relation in $\mathfrak{H}_{2}$. The above decomposition $S=S_{s} \oplus S_{\infty}$ shows that a simple closed symmetric relation is necessarily an operator. Recall that (cf. [26]) a closed symmetric linear relation $S$ in a Hilbert space $\mathfrak{H}$ is simple if and only if

$$
\mathfrak{H}=\overline{\operatorname{span}}\left\{\mathfrak{N}_{\lambda}\left(S^{*}\right): \lambda \in \mathbb{C} \backslash \mathbb{R}\right\} .
$$

2.3. Linear relations in Kre ̌n spaces. Now let $\mathfrak{H}$ and $\mathcal{H}$ be Hilbert spaces and let $j_{\mathfrak{H}}$ and $j_{\mathcal{H}}$ be signature operators in them. Recall that a bounded linear operator $j$ in a Hilbert space is a signature operator if $j=j^{*}=j^{-1}$. Interpret the spaces $\mathfrak{H}$ and $\mathcal{H}$ as Krĕn spaces whose inner products are determined by the fundamental symmetries $j_{\mathfrak{H}}$ and $j_{\mathcal{H}}$. Then the adjoint $T^{[*]}$ of a linear relation $T$ from the Krĕn space $\left(\mathfrak{H}, j_{\mathfrak{H}}\right)$ to the Krein space $\left(\mathcal{H}, j_{\mathcal{H}}\right)$ is given by $T^{[*]}=j_{\mathfrak{H}} T^{*} j_{\mathcal{H}}$. It satisfies the following equalities familiar from the Hilbert space case:

$$
(\operatorname{dom} T)^{[\perp]}=\operatorname{mul} T^{[*]}, \quad(\operatorname{ran} T)^{[\perp]}=\operatorname{ker} T^{[*]} .
$$

Here the orthogonal complements, denoted by $[\perp]$, are with respect to the Krein space structures. The inner products in $\left(\mathfrak{H}, j_{\mathfrak{H}}\right),\left(\mathcal{H}, j_{\mathcal{H}}\right)$ will be denoted by

$$
[\varphi, \psi]_{\mathcal{H}}=\left(j_{\mathfrak{H}} \varphi, \psi\right)_{\mathcal{H}}, \quad\left[\varphi^{\prime}, \psi^{\prime}\right]_{\mathfrak{H}}=\left(j_{\mathfrak{H}} \varphi^{\prime}, \psi^{\prime}\right)_{\mathfrak{H}}, \quad \varphi, \psi \in \mathcal{H}, \quad \varphi^{\prime}, \psi^{\prime} \in \mathfrak{H}
$$

A linear relation $T$ from the Kreĭn space $\left(\mathfrak{H}, j_{\mathfrak{H}}\right)$ to the Krĕn space $\left(\mathcal{H}, j_{\mathcal{H}}\right)$ is called isometric if

$$
\left[\varphi^{\prime}, \varphi^{\prime}\right]_{\mathcal{H}}=[\varphi, \varphi]_{\mathfrak{H}}, \quad\left\{\varphi, \varphi^{\prime}\right\} \in T,
$$

and contractive or expanding if equality in (2.8) is replaced by $\leq$ or by $\geq$, respectively. These properties are invariant under closures. By polarization it follows that a linear relation $T$ is isometric if and only if $T^{-1} \subset T^{[*]}$. A linear relation $T$ is called unitary if $T^{-1}=T^{[*]}$; it is called essentially unitary if $(\operatorname{clos} T)^{-1}=T^{[*]}$.

The first statement in the next proposition is due to Yu.L. Shmul'jan (see [34]), who obtained it by combining [34, Theorem 3] with a result of I.M. Spitkovskiu in 35]. A simple and essentially different proof of this statement is presented below. The second statement is proved in [34, Theorem 2] by using a result of R. Arens [2]. 
Proposition 2.3. Let $T$ be a unitary relation from the Kreun space $\left(\mathfrak{H}, j_{\mathfrak{H}}\right)$ to the Kreı̆n space $\left(\mathcal{H}, j_{\mathcal{H}}\right)$. Then:

(i) $\operatorname{dom} T$ is closed if and only if $\operatorname{ran} T$ is closed;

(ii) the following equalities hold:

$$
\operatorname{ker} T=(\operatorname{dom} T)^{[\perp]}, \quad \operatorname{mul} T=(\operatorname{ran} T)^{[\perp]} .
$$

Proof. By definition $T$ satisfies the identity $T^{-1}=T^{[*]}$, where the KreĬ space adjoint $T^{[*]}$ of $T$ is connected to the Hilbert space adjoint $T^{*}$ via $T^{[*]}=j_{\mathfrak{H}} T^{*} j_{\mathcal{H}}$. It is clear that $\operatorname{dom} T^{[*]}\left(\operatorname{ran} T^{[*]}\right)$ is closed if and only if $\operatorname{dom} T^{*}\left(\operatorname{resp} . \operatorname{ran} T^{*}\right)$ is closed. Therefore, the equivalence $\operatorname{dom} T$ is closed if and only if $\operatorname{ran} T$ is closed follows from Proposition 2.2

To get the identities (2.9) it is enough to apply (2.7) and the equality $T^{-1}=$ $T^{[*]}$.

In the present generality it is useful to give criteria for a unitary relation $T$ : $\left(\mathfrak{H}, j_{\mathfrak{H}}\right) \rightarrow\left(\mathcal{H}, j_{\mathcal{H}}\right)$ to be an operator (not necessarily densely defined).

Corollary 2.4. Let $T$ be a unitary relation from the Krein space $\left(\mathfrak{H}, j_{\mathfrak{H}}\right)$ to the Kreı̆n space $\left(\mathcal{H}, j_{\mathcal{H}}\right)$. Then:

(i) $T$ is single-valued if and only if $\overline{\operatorname{ran}} T=\mathcal{H}$;

(ii) $T$ is a densely defined operator if and only if $\overline{\operatorname{ran}} T=\mathcal{H}$ and $\operatorname{ker} T=\{0\}$;

(iii) $T$ is bounded and single-valued if and only if $\operatorname{ran} T=\mathcal{H}$;

(iv) $T \in[\mathfrak{H}, \mathcal{H}]$ if and only if $\operatorname{ran} T=\mathcal{H}$ and $\operatorname{ker} T=\{0\}$.

Proof. The second identity in (2.9) is $\operatorname{mul} T=(\operatorname{ran} T)^{[\perp]}$, and this gives (i). Moreover, according to Proposition $2.3 \operatorname{ran} T$ is closed if and only if $\operatorname{dom} T$ is closed, and thus (iii) follows from the closed graph theorem. To get (ii) and (iv) it remains to apply the first identity in (2.9) $\operatorname{ker} T=(\operatorname{dom} T)^{[\perp]}$.

Using Krel̆ space terminology, Proposition 2.3 shows that for a unitary relation $T$, the isotropic part of $\operatorname{dom} T$ is equal to $\operatorname{ker} T$ and the isotropic part of $\operatorname{ran} T$ is equal to mul $T$. For an isometric relation $T$ from the Krein space $\left(\mathfrak{H}, j_{\mathfrak{H}}\right)$ to the Kreln space $\left(\mathcal{H}, j_{\mathcal{H}}\right)$, the situation is different. It follows from $T^{-1} \subset T^{[*]}$ and the identities (2.7) that

$$
\operatorname{ker} T \subset(\operatorname{dom} T)^{[\perp]}, \quad \operatorname{mul} T \subset(\operatorname{ran} T)^{[\perp]},
$$

so that $\operatorname{ker} T$ is contained in the isotropic part of $\operatorname{dom} T$ and $\operatorname{mul} T$ is contained in the isotropic part of $\operatorname{ran} T$. It turns out that isometric relations whose domain satisfies the additional property

$$
(\operatorname{dom} T)^{[\perp]} \subset \operatorname{dom} T
$$

play a central role in the construction of boundary mappings. The following results give sufficient conditions for such an isometric relation $T$ to be unitary. The connection to ordinary boundary triplets becomes clear in Section 5 .

Proposition 2.5. Let $T$ be an isometric linear relation from the Krein space $\left(\mathfrak{H}, j_{\mathfrak{H}}\right)$ to the Kreın space $\left(\mathcal{H}, j_{\mathcal{H}}\right)$. If the conditions

(i) $(\operatorname{dom} T)^{[\perp]} \subset \operatorname{dom} T$;

(ii) $(\operatorname{ran} T)^{[\perp]} \subset \operatorname{mul} T$ 
are satisfied, then $T$ also satisfies

$$
\operatorname{ker} T=(\operatorname{dom} T)^{[\perp]} .
$$

Moreover, if the condition (2.11) and the condition

$$
\operatorname{dom} T^{[*]} \subset \operatorname{ran} T
$$

are satisfied, then $T$ is a unitary relation.

Proof. Assume that $f \in(\operatorname{dom} T)^{[\perp]}$ so that $[f, h]_{\mathfrak{H}}=0$ for all $h \in \operatorname{dom} T$. By assumption (i) there exists an element $f^{\prime} \in \mathcal{H}$ so that $\left\{f, f^{\prime}\right\} \in T$. Hence for all $\left\{h, h^{\prime}\right\} \in T$

$$
\left[f^{\prime}, h^{\prime}\right]_{\mathcal{H}}=[f, h]_{\mathfrak{H}}=0,
$$

so that $f^{\prime} \in(\operatorname{ran} T)^{[\perp]}$. Hence, assumption (ii) implies that $f^{\prime} \in \operatorname{mul} T$. Therefore $f \in \operatorname{ker} T$, so that $(\operatorname{dom} T)^{[\perp]} \subset \operatorname{ker} T$. Hence, (2.10) implies that (2.11) is satisfied.

Now assume that (2.11) and (2.12) hold. Let $\left\{f, f^{\prime}\right\} \in T^{[*]}$ so that $\left[f^{\prime}, h\right]_{\mathfrak{H}}=$ $\left[f, h^{\prime}\right]_{\mathcal{H}}$ for all $\left\{h, h^{\prime}\right\} \in T$. The condition (2.12) implies the existence of an element $\varphi \in \mathfrak{H}$ such that $\{\varphi, f\} \in T$. Since $T$ is isometric it follows that $\left[f, h^{\prime}\right]_{\mathcal{H}}=[\varphi, h]_{\mathfrak{H}}$, so that $\left[f^{\prime}, h\right]_{\mathfrak{H}}=[\varphi, h]_{\mathfrak{H}}$ for all $h \in \operatorname{dom} T$. This implies that $f^{\prime}=\varphi+\gamma$ with $\gamma \in(\operatorname{dom} T)^{[\perp]}$. The condition (2.11) shows that $\gamma \in \operatorname{ker} T$. Hence

$$
\left\{f^{\prime}, f\right\}=\{\varphi+\gamma, f\}=\{\varphi, f\}+\{\gamma, 0\} \in T,
$$

which implies that $T^{[*]} \subset T^{-1}$.

Corollary 2.6. Condition (ii) in Proposition 2.5 is automatically satisfied when $\operatorname{ran} T$ is dense in $\mathcal{H}$, in which case $T$ is single-valued. In particular, if (i) holds and $\operatorname{ran} T=\mathcal{H}$, then $\operatorname{dom} T$ is closed and $T$ is a single-valued unitary relation, which is bounded.

Proof. Assume that $\operatorname{ran} T$ is dense in $\mathcal{H}$. Since $(\operatorname{ran} T)^{[\perp]}=\{0\}$, clearly (ii) is satisfied. Since $T$ is isometric it follows from the second inclusion in (2.10) that $T$ is single-valued.

Now assume $\operatorname{ran} T=\mathcal{H}$. Hence, $\operatorname{ran} T$ is dense in $\mathcal{H}$, so that (ii) follows and (2.11) automatically follows. Moreover, (2.12) is also automatically satisfied, so that (ii) implies that $T$ is unitary. It follows from Proposition 2.3 that $\operatorname{dom} T$ is closed. The boundedness of $T$ follows from the closed graph theorem.

Clearly, with $T$ the inverse $T^{-1}$ is also isometric. Hence, a formal inversion in Proposition 2.5 gives the following equivalent version.

Proposition 2.7. Let $T$ be an isometric linear relation from the Krein space $\left(\mathfrak{H}, j_{\mathfrak{H}}\right)$ to the Kre ̌n space $\left(\mathcal{H}, j_{\mathcal{H}}\right)$. If the conditions

(i) $(\operatorname{ran} T)^{[\perp]} \subset \operatorname{ran} T$;

(ii) $(\operatorname{dom} T)^{[\perp]} \subset \operatorname{ker} T$

are satisfied, then $T$ also satisfies

$$
\operatorname{mul} T=(\operatorname{ran} T)^{[\perp]} .
$$

Moreover, if the condition (2.13) and the condition

$$
\operatorname{ran} T^{[*]} \subset \operatorname{dom} T
$$

are satisfied, then $T$ is a unitary relation. 
Corollary 2.8. Condition (ii) in Proposition 2.7 is automatically satisfied when $\operatorname{dom} T$ is dense in $\mathcal{H}$, in which case $T^{-1}$ is single-valued. In particular, if (i) holds and $\operatorname{dom} T=\mathcal{H}$, then $\operatorname{ran} T$ is closed and $T^{-1}$ is a single-valued unitary relation, which is bounded.

Since with $T$ the closure of $T$ is also isometric, it is possible to replace in Propositions 2.5, 2.7, and their corollaries the relation $T$ by its closure to conclude that $T$ is an essentially unitary relation.

Let $T$ be an isometric operator from the Krein space $\left(\mathfrak{H}, j_{\mathfrak{H}}\right)$ to the Kreı̆ space $\left(\mathcal{H}, j_{\mathcal{H}}\right)$ such that $\overline{\operatorname{dom}} T=\mathfrak{H}$ and $\overline{\operatorname{ran}} T=\mathcal{H}$. If either (2.12) or (2.14) holds, then $T$ and $T^{-1}$ are single-valued unitary relations, which are in general unbounded; see Examples 6.5, 6.6. In particular, if either $\overline{\operatorname{dom}} T=\mathfrak{H}$ and $\operatorname{ran} T=\mathcal{H}$, or $\operatorname{dom} T=\mathfrak{H}$ and $\operatorname{ran} T=\mathcal{H}$, then by Corollary 2.6 or 2.8 both $T$ and $T^{-1}$ are unitary operators, which are bounded (cf. [3, Chapter 2, Definition 5.1 and Corollary 5.8]).

In what follows it is convenient to interpret the Hilbert space $\mathfrak{H}^{2}=\mathfrak{H} \oplus \mathfrak{H}$ as a Kreŭ space $\left(\mathfrak{H}^{2}, J_{\mathfrak{H}}\right)$ whose inner product is determined by the fundamental symmetry $J_{\mathfrak{H}}$ :

$$
J_{\mathfrak{H}}:=\left(\begin{array}{cc}
0 & -i I_{\mathfrak{H}} \\
i I_{\mathfrak{H}} & 0
\end{array}\right)
$$

The adjoint $T^{*}$ in (2.3) of a linear relation $T$ in the Hilbert space $\mathfrak{H}$ can be written in terms of $J_{\mathfrak{H}}$ as

$$
T^{*}=J_{\mathfrak{H}} T^{\perp}=\left(J_{\mathfrak{H}} T\right)^{\perp} .
$$

The following connections between linear relations in the Hilbert space $\mathfrak{H}$ and subspaces in the Kreйn space $\left(\mathfrak{H}^{2}, J_{\mathfrak{H}}\right)$ will be useful.

Proposition $2.9([3],[32])$. Let $T$ be a linear relation in the Hilbert space $\mathfrak{H}$. Then

(i) $T$ is symmetric (selfadjoint) if and only if $T$ is a neutral (hypermaximal neutral) subspace of $\left(\mathfrak{H}^{2}, J_{\mathfrak{H}}\right)$;

(ii) $T$ is dissipative (maximal dissipative) if and only if $T$ is a nonnegative (maximal nonnegative) subspace of $\left(\mathfrak{H}^{2}, J_{\mathfrak{H}}\right)$;

(iii) $T$ is accumulative (maximal accumulative) if and only if $T$ is a nonpositive (maximal nonpositive) subspace of $\left(\mathfrak{H}^{2}, J_{\mathfrak{H}}\right)$.

2.4. The main transform. Let $\mathfrak{H}$ and $\mathcal{H}$ be Hilbert spaces and let their Cartesian product be denoted by $\widetilde{\mathfrak{H}}=\mathfrak{H} \oplus \mathcal{H}$. Define the linear mapping $\mathcal{J}$ from $\mathfrak{H}^{2} \times \mathcal{H}^{2}$ to $(\mathfrak{H} \oplus \mathcal{H})^{2}$ by

$$
\mathcal{J}:\left\{\left(\begin{array}{c}
f \\
f^{\prime}
\end{array}\right),\left(\begin{array}{c}
h \\
h^{\prime}
\end{array}\right)\right\} \mapsto\left\{\left(\begin{array}{c}
f \\
h
\end{array}\right),\left(\begin{array}{c}
f^{\prime} \\
-h^{\prime}
\end{array}\right)\right\}, \quad f, f^{\prime} \in \mathfrak{H}, h, h^{\prime} \in \mathcal{H} .
$$

This mapping establishes a one-to-one correspondence between the (closed) linear relations $\Gamma: \mathfrak{H}^{2} \rightarrow \mathcal{H}^{2}$ and the (closed) linear relations $\widetilde{A}$ in $\widetilde{\mathfrak{H}}=\mathfrak{H} \oplus \mathcal{H}$ via

$$
\Gamma \mapsto \widetilde{A}:=\mathcal{J}(\Gamma)=\left\{\left\{\left(\begin{array}{c}
f \\
h
\end{array}\right),\left(\begin{array}{c}
f^{\prime} \\
-h^{\prime}
\end{array}\right)\right\}:\left\{\left(\begin{array}{c}
f \\
f^{\prime}
\end{array}\right),\left(\begin{array}{c}
h \\
h^{\prime}
\end{array}\right)\right\} \in \Gamma\right\} .
$$

The mapping $\mathcal{J}$ plays a principal role in the whole paper, and it is referred to as the main transform. Some basic properties of this transform are stated in the following proposition. 
Proposition 2.10. Let the linear relation $\Gamma$ from $\left(\mathfrak{H}^{2}, J_{\mathfrak{H}}\right)$ to $\left(\mathcal{H}^{2}, J_{\mathcal{H}}\right)$ and the linear relation $\widetilde{A}$ in $\mathfrak{H} \oplus \mathcal{H}$ be connected by $\widetilde{A}=\mathcal{J}(\Gamma)$. Then

$$
\widetilde{A}^{*}=\mathcal{J}\left(\left(\Gamma^{[*]}\right)^{-1}\right) .
$$

Moreover, the transform $\mathcal{J}$ establishes a one-to-one correspondence between the isometric (contractive, expanding, unitary) relations $\Gamma$ from $\left(\mathfrak{H}^{2}, J_{\mathfrak{H}}\right)$ to $\left(\mathcal{H}^{2}, J_{\mathcal{H}}\right)$ and the symmetric (dissipative, accumulative, selfadjoint) relations in $\mathfrak{H} \oplus \mathcal{H}$.

Proof. It is straightforward to check that for all elements of the form

$$
\left\{\left(\begin{array}{c}
f \\
f^{\prime}
\end{array}\right),\left(\begin{array}{c}
h \\
h^{\prime}
\end{array}\right)\right\},\left\{\left(\begin{array}{c}
g \\
g^{\prime}
\end{array}\right),\left(\begin{array}{c}
k \\
k^{\prime}
\end{array}\right)\right\} \in\left(\begin{array}{c}
\mathfrak{H} \\
\mathfrak{H}
\end{array}\right) \times\left(\begin{array}{c}
\mathcal{H} \\
\mathcal{H}
\end{array}\right),
$$

the following identity is satisfied:

$$
\begin{aligned}
& \frac{1}{i}\left\{\left(\left(\begin{array}{c}
f^{\prime} \\
-h^{\prime}
\end{array}\right),\left(\begin{array}{l}
g \\
k
\end{array}\right)\right)_{\mathfrak{H} \oplus \mathcal{H}}-\left(\left(\begin{array}{c}
f \\
h
\end{array}\right),\left(\begin{array}{c}
g^{\prime} \\
-k^{\prime}
\end{array}\right)\right)_{\mathfrak{H} \oplus \mathcal{H}}\right\} \\
& =\left(J_{\mathfrak{H}}\left(\begin{array}{c}
f \\
f^{\prime}
\end{array}\right),\left(\begin{array}{c}
g \\
g^{\prime}
\end{array}\right)\right)_{\mathfrak{H}^{2}}-\left(J_{\mathcal{H}}\left(\begin{array}{c}
h \\
h^{\prime}
\end{array}\right),\left(\begin{array}{c}
k \\
k^{\prime}
\end{array}\right)\right)_{\mathcal{H}^{2}} .
\end{aligned}
$$

This identity implies the equivalence

$$
\left\{\left(\begin{array}{c}
f \\
h
\end{array}\right),\left(\begin{array}{c}
f^{\prime} \\
-h^{\prime}
\end{array}\right)\right\} \in \widetilde{A}^{*} \quad \Leftrightarrow \quad\left\{\left(\begin{array}{c}
h \\
h^{\prime}
\end{array}\right),\left(\begin{array}{c}
f \\
f^{\prime}
\end{array}\right)\right\} \in \Gamma^{[*]}
$$

which leads to the identity (2.17). Hence it follows that

$$
\widetilde{A} \subset \widetilde{A}^{*} \quad \Leftrightarrow \quad \Gamma^{-1} \subset \Gamma^{[*]}, \quad \widetilde{A}=\widetilde{A}^{*} \Leftrightarrow \Gamma^{-1}=\Gamma^{[*]} .
$$

Observe that (2.18) in particular leads to the following identity:

$$
2 \operatorname{Im}\left(\left(\begin{array}{c}
f^{\prime} \\
-h^{\prime}
\end{array}\right),\left(\begin{array}{c}
f \\
h
\end{array}\right)\right)_{\mathfrak{H} \oplus \mathcal{H}}=\left(J_{\mathfrak{H}}\left(\begin{array}{c}
f \\
f^{\prime}
\end{array}\right),\left(\begin{array}{c}
f \\
f^{\prime}
\end{array}\right)\right)_{\mathfrak{H}^{2}}-\left(J_{\mathcal{H}}\left(\begin{array}{c}
h \\
h^{\prime}
\end{array}\right),\left(\begin{array}{c}
h \\
h^{\prime}
\end{array}\right)\right)_{\mathcal{H}^{2}} .
$$

This implies the connection between the contractive (expanding) relations $\Gamma$ from $\left(\mathfrak{H}^{2}, J_{\mathfrak{H}}\right)$ to $\left(\mathcal{H}^{2}, J_{\mathcal{H}}\right)$ and the dissipative (accumulative) relations $\widetilde{A}$ in $\mathfrak{H} \oplus \mathcal{H}$.

Remark 2.11. Let $\mathcal{C}$ be a Cayley transform of $\widetilde{A}$ :

$$
\mathcal{C}: \widetilde{A} \mapsto U=\left\{\left\{u^{\prime}+i u, u^{\prime}-i u\right\}:\left\{u, u^{\prime}\right\} \in \widetilde{A}\right\} .
$$

Then the transform $\mathcal{C} \circ \mathcal{J}$ is a kind of Potapov-Ginzburg transform (see 34]) which establishes a one-to-one correspondence between isometric (contractive, expanding, unitary) relations from $\left(\mathfrak{H}^{2}, J_{\mathfrak{H}}\right)$ to $\left(\mathcal{H}^{2}, J_{\mathcal{H}}\right)$ and isometric (contractive, expanding, unitary) operators in $\mathfrak{H} \oplus \mathcal{H}$.

With the Hilbert spaces $\mathfrak{H}$ and $\mathcal{H}$ the Cartesian product $\widetilde{\mathfrak{H}}=\mathfrak{H} \oplus \mathcal{H}$ has been defined. The following identifications will be used:

$$
\mathfrak{H}_{1}=\left(\begin{array}{c}
\mathfrak{H} \\
0
\end{array}\right), \quad \mathfrak{H}_{2}=\left(\begin{array}{c}
0 \\
\mathcal{H}
\end{array}\right), \quad \widetilde{\mathfrak{H}}=\mathfrak{H}_{1} \oplus \mathfrak{H}_{2}=\left(\begin{array}{c}
\mathfrak{H} \\
\mathcal{H}
\end{array}\right) .
$$

Let $P_{j}$ be the orthogonal projection from $\widetilde{\mathfrak{H}}$ onto $\mathfrak{H}_{j}, j=1,2$. 
Proposition 2.12. Let the linear relations $\Gamma$ and $\widetilde{A}$ be related by (2.16), i.e. $\widetilde{A}=$ $\mathcal{J}(\Gamma)$. Then the linear relations

$$
S_{1}=\operatorname{ker} \Gamma, \quad-S_{2}=\operatorname{mul} \Gamma, \quad T_{1}=\operatorname{dom} \Gamma, \quad-T_{2}=\operatorname{ran} \Gamma,
$$

are given by

$$
S_{j}=\widetilde{A} \cap \mathfrak{H}_{j}^{2}, \quad T_{j}=\left\{\left\{P_{j} \varphi, P_{j} \varphi^{\prime}\right\}:\left\{\varphi, \varphi^{\prime}\right\} \in \widetilde{A}\right\} .
$$

Moreover, if $\widetilde{A}$ is a symmetric linear relation in $\widetilde{\mathfrak{H}}$, then $T_{j} \subset S_{j}^{*}$ and in particular $S_{j}$ is a symmetric linear relation in $\mathfrak{H}_{j}, j=1,2$. If, in addition, $\widetilde{A}$ is selfadjoint, then

$$
\operatorname{clos} T_{j}=S_{j}^{*} \quad j=1,2 .
$$

Proof. The equalities (2.21) are immediate from (2.16). The inclusions $T_{j} \subset S_{j}^{*}$ with $\widetilde{A}$ symmetric ( $\Gamma$ isometric) and the equalities (2.22) with $\widetilde{A}$ selfadjoint $(\Gamma$ unitary) are implied by Proposition 2.3 in view of (2.20) and (2.15).

If for $j=1$ or $j=2$, the relation $S_{j}$ is densely defined, then it follows from (2.22) that $\cos T_{j}=S_{j}^{*}$ is an operator, and (2.21) shows that $P_{j}$ mul $\widetilde{A}=\{0\}$.

The next result gives some mapping properties of isometric relations in product spaces.

Proposition 2.13. Let $\Gamma$ be an isometric relation from $\left(\mathfrak{H}^{2}, J_{\mathfrak{H}}\right)$ to $\left(\mathcal{H}^{2}, J_{\mathcal{H}}\right)$ and let $A \subset \operatorname{dom} \Gamma$ be a linear relation in $\mathfrak{H}^{2}$. Then:

(i) $A$ is symmetric (dissipative, accumulative) in $\mathfrak{H}^{2}$ if and only if $\Gamma(A)$ is symmetric (dissipative, accumulative) in $\mathcal{H}^{2}$;

(ii) if $A^{*} \subset \operatorname{dom} \Gamma$, then $\Gamma\left(A^{*}\right) \subset \Gamma(A)^{*}$;

(iii) if $A^{*} \subset \operatorname{dom} \Gamma$ and $\Gamma(A)$ is essentially selfadjoint in $\mathcal{H}^{2}$, then $A$ is essentially selfadjoint in $\mathfrak{H}^{2}$.

Proof. (i) By definition $\Gamma(A)=\{\widehat{h}:\{\widehat{f}, \widehat{h}\} \in \Gamma$ for some $\widehat{f} \in A\}$, and the statement follows from

$$
2 \operatorname{Im}\left(f^{\prime}, f\right)=\left(J_{\mathfrak{H}}\left(\begin{array}{c}
f \\
f^{\prime}
\end{array}\right),\left(\begin{array}{c}
f \\
f^{\prime}
\end{array}\right)\right)_{\mathfrak{H}^{2}}=\left(J_{\mathcal{H}}\left(\begin{array}{c}
h \\
h^{\prime}
\end{array}\right),\left(\begin{array}{c}
h \\
h^{\prime}
\end{array}\right)\right)_{\mathcal{H}^{2}}=2 \operatorname{Im}\left(h^{\prime}, h\right) .
$$

(ii) Let $\widehat{g} \in A^{*}$ and let $\{\widehat{g}, \widehat{k}\} \in \Gamma$. Then for every $\widehat{h} \in \Gamma(A)$ one obtains

$$
0=\left(J_{\mathfrak{H}}\left(\begin{array}{c}
g \\
g^{\prime}
\end{array}\right),\left(\begin{array}{c}
f \\
f^{\prime}
\end{array}\right)\right)_{\mathfrak{H}^{2}}=\left(J_{\mathcal{H}}\left(\begin{array}{c}
k \\
k^{\prime}
\end{array}\right),\left(\begin{array}{c}
h \\
h^{\prime}
\end{array}\right)\right)_{\mathcal{H}^{2}},
$$

since here $\widehat{f} \in A$. This means that $\widehat{k} \in \Gamma(A)^{*}$ and hence $\Gamma\left(A^{*}\right) \subset \Gamma(A)^{*}$.

(iii) If $\Gamma(A)$ is essentially selfadjoint, then by part (i) $A$ is symmetric. Now part (ii) shows that $\Gamma(A) \subset \Gamma\left(A^{*}\right) \subset \Gamma(A)^{*}$. Hence, $\operatorname{clos} \Gamma(A)=\operatorname{clos} \Gamma\left(A^{*}\right)$ and $\Gamma\left(A^{*}\right)$ is essentially selfadjoint. Therefore, $A^{*}$ must be symmetric by part (i), and hence $A^{*}=A^{* *}=\operatorname{clos} A$.

2.5. Orthogonal couplings. Let $\mathfrak{H}_{1}$ and $\mathfrak{H}_{2}$ be arbitrary Hilbert spaces (not necessarily the same as in (2.19) ) and let $\widetilde{A}$ be a selfadjoint linear relation in the orthogonal sum $\widetilde{\mathfrak{H}}=\mathfrak{H}_{1} \oplus \mathfrak{H}_{2}$. Then the formula (2.21) defines closed symmetric linear relations $S_{1}$ and $S_{2}$, and not necessarily closed linear relations $T_{1}$ and $T_{2}$, in $\mathfrak{H}_{1}$ and $\mathfrak{H}_{2}$, respectively. The relation $\widetilde{A}$ can be interpreted as a selfadjoint extension of the orthogonal sum $S_{1} \oplus S_{2}$. It is called the orthogonal coupling of $S_{1}$ and 
$T_{2}$ (or of $T_{1}$ and $S_{2}$ ); see [38. The selfadjoint relation $\widetilde{A}$ is said to be minimal with respect to the Hilbert space $\mathfrak{H}_{j}(j$ is fixed, $j=1,2)$ if

$$
\mathfrak{H}_{1} \oplus \mathfrak{H}_{2}=\overline{\operatorname{span}}\left\{\mathfrak{H}_{j}+(\widetilde{A}-\lambda)^{-1} \mathfrak{H}_{j}: \lambda \in \rho(\widetilde{A})\right\} .
$$

The null spaces of $T_{j}-\lambda$ as in (2.2) are said to be "defect spaces" of the linear relations $T_{j}$, i.e.,

$$
\mathfrak{N}_{\lambda}\left(T_{j}\right)=\operatorname{ker}\left(T_{j}-\lambda\right), \quad \widehat{\mathfrak{N}}_{\lambda}\left(T_{j}\right)=\left\{\{f, \lambda f\} \in T_{j}: f \in \mathfrak{N}_{\lambda}\left(T_{j}\right)\right\} .
$$

For notational convenience the usual defect spaces of $S_{j}$ are denoted here by $\mathfrak{N}_{\lambda}\left(S_{j}^{*}\right)$ and $\widehat{\mathfrak{N}}_{\lambda}\left(S_{j}^{*}\right)$.

Lemma 2.14. Let $\widetilde{A}$ be a selfadjoint linear relation in $\widetilde{\mathfrak{H}}=\mathfrak{H}_{1} \oplus \mathfrak{H}_{2}$, and let the linear relations $S_{j}$ and $T_{j}, j=1,2$, be defined by (2.21). Then:

(i) $\mathfrak{N}_{\lambda}\left(T_{1}\right)=P_{1}(\widetilde{A}-\lambda)^{-1} \mathfrak{H}_{2}, \mathfrak{N}_{\lambda}\left(T_{2}\right)=P_{2}(\widetilde{A}-\lambda)^{-1} \mathfrak{H}_{1}$;

(ii) $\mathfrak{N}_{\lambda}\left(T_{j}\right)$ is dense in $\mathfrak{N}_{\lambda}\left(S_{j}^{*}\right)$ for all $\lambda \in \mathbb{C} \backslash \mathbb{R}, j=1,2$;

(iii) the defect numbers of $S_{1}$ and $-S_{2}$ coincide: $n_{ \pm}\left(S_{1}\right)=n_{\mp}\left(S_{2}\right)$;

(iv) $\widetilde{A}$ is minimal with respect to $\mathfrak{H}_{1}$ (resp. $\mathfrak{H}_{2}$ ) if and only if $S_{2}$ (resp. $S_{1}$ ) is simple.

Proof. First observe that

$$
(\widetilde{A}-\lambda)^{-1}\left(\begin{array}{c}
f^{\prime}-\lambda f \\
-h^{\prime}-\lambda h
\end{array}\right)=\left(\begin{array}{l}
f \\
h
\end{array}\right), \quad\left\{\left(\begin{array}{l}
f \\
h
\end{array}\right),\left(\begin{array}{c}
f^{\prime} \\
-h^{\prime}
\end{array}\right)\right\} \in \widetilde{A}
$$

(i) Note in (2.25) that $f \in \mathfrak{N}_{\lambda}\left(T_{1}\right)$ if and only if $f^{\prime}=\lambda f$. This gives the first assertion. The proof of the second assertion is similar.

(ii) Using $\operatorname{ran}\left(S_{1}-\lambda\right)=\left\{f^{\prime}-\lambda f: f \in \operatorname{dom} S_{1}\right\}$ one obtains from (2.25) the identities

(2.26) $\operatorname{ker} P_{2}(\widetilde{A}-\lambda)^{-1} \uparrow \mathfrak{H}_{1}=\operatorname{ran}\left(S_{1}-\lambda\right), \quad \operatorname{ker} P_{1}(\widetilde{A}-\lambda)^{-1}\left\lceil\mathfrak{H}_{2}=\operatorname{ran}\left(S_{2}-\lambda\right)\right.$.

Note that $\overline{\operatorname{ran}} X^{*}=(\operatorname{ker} X)^{\perp}$ for any bounded linear operator $X$. Thus the identities in (2.26) imply that the ranges of

$$
\begin{aligned}
& P_{1}(\widetilde{A}-\lambda)^{-1}\left\lceil\mathfrak{H}_{2}=\left(P_{2}(\widetilde{A}-\bar{\lambda})^{-1}\left\lceil\mathfrak{H}_{1}\right)^{*},\right.\right. \\
& P_{2}(\widetilde{A}-\lambda)^{-1}\left\lceil\mathfrak{H}_{1}=\left(P_{1}(\widetilde{A}-\bar{\lambda})^{-1}\left\lceil\mathfrak{H}_{2}\right)^{*}\right.\right.
\end{aligned}
$$

are dense subsets of $\mathfrak{N}_{\lambda}\left(S_{1}^{*}\right)$ and $\mathfrak{N}_{\lambda}\left(S_{2}^{*}\right)$ for all $\lambda \in \mathbb{C}_{+} \cup \mathbb{C}_{-}$, respectively.

(iii) In view of (2.26) the statements (i) and (ii) can be rewritten in the form

$$
\operatorname{clos} P_{1}(\widetilde{A}-\lambda)^{-1} \mathfrak{N}_{\bar{\lambda}}\left(S_{2}^{*}\right)=\mathfrak{N}_{\lambda}\left(S_{1}^{*}\right), \quad \operatorname{clos} P_{2}(\widetilde{A}-\lambda)^{-1} \mathfrak{N}_{\bar{\lambda}}\left(S_{1}^{*}\right)=\mathfrak{N}_{\lambda}\left(S_{2}^{*}\right),
$$

respectively. These identities imply the equality of the defect numbers.

(iv) If $\widetilde{A}$ is minimal with respect to $\mathfrak{H}_{1}$, then it follows from (i), (ii), and (2.23) that

$$
\mathfrak{H}_{2}=\overline{\operatorname{span}}\left\{P_{2}(\widetilde{A}-\lambda)^{-1} \mathfrak{H}_{1}: \lambda \in \rho(\widetilde{A})\right\}=\overline{\operatorname{span}}\left\{\mathfrak{N}_{\lambda}\left(S_{2}^{*}\right): \lambda \in \rho(\widetilde{A})\right\},
$$

so that $S_{2}$ is simple. Conversely, if $S_{2}$ is simple, then clearly (2.23) is satisfied and $\widetilde{A}$ is minimal with respect to $\mathfrak{H}_{1}$.

Proposition 2.15. Let $\widetilde{A}$ be a selfadjoint relation in $\widetilde{\mathfrak{H}}=\mathfrak{H}_{1} \oplus \mathfrak{H}_{2}$ and let the linear relations $T_{1}$ and $T_{2}$ be given by (2.21). Then $T_{1}$ is closed if and only if $T_{2}$ is closed. 
Proof. Let $\Gamma$ be defined by $\widetilde{A}=\mathcal{J}(\Gamma)$, so that $\Gamma$ is a unitary relation. By definition $T_{1}=\operatorname{dom} \Gamma$ and $T_{2}=\operatorname{ran} \Gamma$. Hence, the statement follows from Proposition 2.3.

2.6. Nevanlinna families. A family of linear relations $M(\lambda), \lambda \in \mathbb{C} \backslash \mathbb{R}$, in a Hilbert space $\mathcal{H}$ is called a Nevanlinna family if:

(i) for every $\lambda \in \mathbb{C}_{+}\left(\mathbb{C}_{-}\right)$the relation $M(\lambda)$ is maximal dissipative (resp. accumulative);

(ii) $M(\lambda)^{*}=M(\bar{\lambda}), \lambda \in \mathbb{C} \backslash \mathbb{R}$;

(iii) for some, and hence for all, $\mu \in \mathbb{C}_{+}\left(\mathbb{C}_{-}\right)$the operator family $(M(\lambda)+\mu)^{-1}$ $(\in[\mathcal{H}])$ is holomorphic for all $\lambda \in \mathbb{C}_{+}\left(\mathbb{C}_{-}\right)$.

By the maximality condition, each relation $M(\lambda), \lambda \in \mathbb{C} \backslash \mathbb{R}$, is necessarily closed. The class of all Nevanlinna families in a Hilbert space is denoted by $\widetilde{R}(\mathcal{H})$. Nevanlinna families were considered in [24, [17, and [26]. The following orthogonal decomposition is useful.

Proposition 2.16. If $M(\cdot) \in \widetilde{R}(\mathcal{H})$, then the multi-valued part mul $M(\lambda)$ is independent of $\lambda \in \mathbb{C} \backslash \mathbb{R}$, so that

$$
M(\lambda)=M_{s}(\lambda) \oplus M_{\infty}, \quad M_{\infty}=\{0\} \times \operatorname{mul} M(\lambda), \quad \lambda \in \mathbb{C} \backslash \mathbb{R},
$$

where $M_{s}(\lambda)$ is a Nevanlinna family of densely defined operators in $\mathcal{H} \ominus$ mul $M(\lambda)$.

Clearly, if $M(\cdot) \in \widetilde{R}(\mathcal{H})$, then $M_{\infty} \subset M(\lambda) \cap M(\lambda)^{*}$ for all $\lambda \in \mathbb{C} \backslash \mathbb{R}$. The following subclasses of the class $\widetilde{R}(\mathcal{H})$ will be useful:

$R(\mathcal{H})$ is the set of all $M(\cdot) \in \widetilde{R}(\mathcal{H})$ for which mul $M(\lambda)=\{0\} ;$

$R^{s}(\mathcal{H})$ is the set of all $M(\cdot) \in \widetilde{R}(\mathcal{H})$ for which $M(\lambda) \cap M(\lambda)^{*}=\{0\}$ for all $\lambda \in \mathbb{C} \backslash \mathbb{R}$

$R^{u}(\mathcal{H})$ is the set of all $M(\cdot) \in \widetilde{R}(\mathcal{H})$ for which $M(\lambda) \hat{+} M(\lambda)^{*}=\mathcal{H}^{2}$ for all $\lambda \in \mathbb{C} \backslash \mathbb{R}$.

Hence, $M(\cdot) \in R^{s}(\mathcal{H})$ or $M(\cdot) \in R^{u}(\mathcal{H})$, if $M(\lambda)$ and $M(\lambda)^{*}$ are disjoint or transversal, respectively, for every $\lambda \in \mathbb{C} \backslash \mathbb{R}$. With the classes $\widetilde{R}(\mathcal{H}), R(\mathcal{H}), R^{s}(\mathcal{H})$, and $R^{u}(\mathcal{H})$ correspond the classes $\widetilde{R}_{\text {inv }}(\mathcal{H}), R_{\text {inv }}(\mathcal{H}), R_{\text {inv }}^{s}(\mathcal{H})$, and $R_{\text {inv }}^{u}(\mathcal{H})$ of Nevanlinna families $M(\cdot)$ whose domain $\operatorname{dom} M(\lambda)$ does not depend on $\lambda \in \mathbb{C} \backslash \mathbb{R}$. Furthermore, the following subclasses of $\widetilde{R}(\mathcal{H})$ will be important:

$\widetilde{R}[\mathcal{H}]$ is the set of all $M(\cdot) \in \widetilde{R}(\mathcal{H})$ for which $\operatorname{dom} M(\lambda)$ is closed for all $\lambda \in \mathbb{C} \backslash \mathbb{R}$

$R[\mathcal{H}]$ is the set of all $M(\cdot) \in \widetilde{R}[\mathcal{H}]$ for which $\operatorname{dom} M(\lambda)=\mathcal{H}$ for all $\lambda \in$ $\mathbb{C} \backslash \mathbb{R}$

$R^{s}[\mathcal{H}]$ is the set of all $M(\cdot) \in R[\mathcal{H}]$ for which $\operatorname{ker} \operatorname{Im} M(\lambda)=\{0\}$ for all $\lambda \in \mathbb{C} \backslash \mathbb{R}$

$R^{u}[\mathcal{H}]$ is the set of all $M(\cdot) \in R^{s}[\mathcal{H}]$ for which $0 \in \rho(\operatorname{Im} M(\lambda))$ for all $\lambda \in \mathbb{C} \backslash \mathbb{R}$.

Remark 2.17. In Section 4 it will be shown that various properties which were used above to define the different subclasses of Nevanlinna families do not depend on $\lambda \in \mathbb{C} \backslash \mathbb{R}$. This means that the corresponding subclasses of $\widetilde{R}(\mathcal{H})$ can be equivalently defined by assuming the corresponding property of $M(\lambda)$ only at a single point $\lambda \in \mathbb{C} \backslash \mathbb{R}$. 
For each $M(\cdot)$ in $\widetilde{R}[\mathcal{H}]$ (or in its subclasses) the operator $M_{s}(\lambda)$ is necessarily bounded. In what follows, the Nevanlinna functions in $R^{s}[\mathcal{H}]$ and $R^{u}[\mathcal{H}]$ will be called strict and uniformly strict, respectively.

Proposition 2.18. Let $M(\cdot) \in \widetilde{R}(\mathcal{H})$. Then the following statements are equivalent:

(i) $M(\cdot) \in R^{u}(\mathcal{H})$;

(ii) $M(\lambda) \in[\mathcal{H}]$ and $0 \in \rho(\operatorname{Im} M(\lambda))$ for some, and hence for all, $\lambda \in \mathbb{C} \backslash \mathbb{R}$.

The result in Proposition 2.18 is a consequence of Propositions 4.5] and 5.3. see also Theorem 4.13 and Remark 4.23

The definitions and Proposition 2.18 give rise to the inclusions and the equalities in the following array:

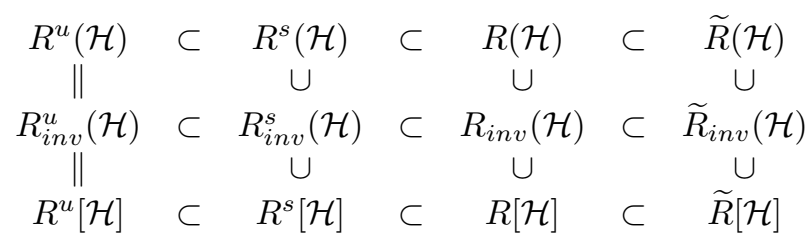

In the infinite-dimensional situation each of the inclusions is strict. However, in the finite-dimensional situation the vertical inclusions in (2.28) reduce to equalities

$$
R^{s}(\mathcal{H})=R_{\text {inv }}^{s}(\mathcal{H})=R^{s}[\mathcal{H}], R(\mathcal{H})=R_{\text {inv }}(\mathcal{H})=R[\mathcal{H}], \widetilde{R}(\mathcal{H})=\widetilde{R}_{\text {inv }}(\mathcal{H})=\widetilde{R}[\mathcal{H}] .
$$

If $M(\cdot) \in R[\mathcal{H}]$, then it admits the following integral representation:

$$
M(\lambda)=A+B \lambda+\int_{\mathbb{R}}\left(\frac{1}{t-\lambda}-\frac{t}{t^{2}+1}\right) d \Sigma(t), \quad \int_{\mathbb{R}} \frac{d \Sigma(t)}{t^{2}+1} \in[\mathcal{H}],
$$

where $A=A^{*} \in[\mathcal{H}], 0 \leq B=B^{*} \in[\mathcal{H}]$, the $[\mathcal{H}]$-valued family $\Sigma(\cdot)$ is nondecreasing, and the integral is uniformly convergent in the strong topology; cf. 7], 21].

\section{Boundary RELATIONS AND WEYL FAMILIES}

3.1. Definition of a boundary relation and its Weyl family. Let $S$ be a closed symmetric linear relation in the Hilbert space $\mathfrak{H}$. It is not assumed that the defect numbers of $S$ are equal or finite. A boundary relation for $S^{*}$ is defined as follows.

Definition 3.1. Let $S$ be a closed symmetric linear relation in a Hilbert space $\mathfrak{H}$ and let $\mathcal{H}$ be an auxiliary Hilbert space. A linear relation $\Gamma: \mathfrak{H}^{2} \mapsto \mathcal{H}^{2}$ is called a boundary relation for $S^{*}$, if:

(G1) dom $\Gamma$ is dense in $S^{*}$, and the identity

$$
\left(f^{\prime}, g\right)_{\mathfrak{H}}-\left(f, g^{\prime}\right)_{\mathfrak{H}}=\left(h^{\prime}, k\right)_{\mathcal{H}}-\left(h, k^{\prime}\right)_{\mathcal{H}}
$$

holds for every $\{\widehat{f}, \widehat{h}\},\{\widehat{g}, \widehat{k}\} \in \Gamma$;

(G2) $\Gamma$ is maximal in the sense that if $\{\widehat{g}, \widehat{k}\} \in \mathfrak{H}^{2} \times \mathcal{H}^{2}$ satisfies (3.1) for every $\{\widehat{f}, \widehat{h}\} \in \Gamma$, then $\{\widehat{g}, \widehat{k}\} \in \Gamma$.

Here $\widehat{f}=\left\{f, f^{\prime}\right\}, \widehat{g}=\left\{g, g^{\prime}\right\} \in \operatorname{dom} \Gamma\left(\subset \mathfrak{H}^{2}\right)$, and $\widehat{h}=\left\{h, h^{\prime}\right\}, \widehat{k}=\left\{k, k^{\prime}\right\} \in$ $\left.\operatorname{ran} \Gamma\left(\subset \mathcal{H}^{2}\right)\right)$. 
The condition (3.1) in (G1) can be interpreted as an abstract Green's identity. Using the terminology of Kreln spaces (3.1) means that $\Gamma$ is an isometric relation from the Krel̆n space $\left(\mathfrak{H}^{2}, J_{\mathfrak{H}}\right)$ to the Krel̆n space $\left(\mathcal{H}^{2}, J_{\mathcal{H}}\right)$, since

$$
\left(J_{\mathfrak{H}} \widehat{f}, \widehat{g}\right)_{\mathfrak{H}^{2}}=\left(J_{\mathcal{H}} \widehat{h}, \widehat{k}\right)_{\mathcal{H}^{2}}, \quad\{\widehat{f}, \widehat{h}\}, \quad\{\widehat{g}, \widehat{k}\} \in \Gamma .
$$

The maximality condition (G2) and Proposition 2.3 now imply the following result.

Proposition 3.2. Let $\Gamma: \mathfrak{H}^{2} \mapsto \mathcal{H}^{2}$ be a boundary relation for $S^{*}$. Then $\Gamma$ is a unitary relation from the Krein space $\left(\mathfrak{H}^{2}, J_{\mathfrak{H}}\right)$ to the Kreĭn space $\left(\mathcal{H}^{2}, J_{\mathcal{H}}\right)$. Moreover, $S=\operatorname{ker} \Gamma$.

Proof. In view of (3.2) $\Gamma$ is isometric, i.e., $\Gamma^{-1} \subset \Gamma^{[*]}$. Now assume that $\{\widehat{k}, \widehat{g}\} \in$ $\Gamma^{[*]}$. Then

$$
\left(J_{\mathfrak{H}} \widehat{g}, \widehat{f}\right)_{\mathfrak{H}^{2}}-\left(J_{\mathcal{H}} \widehat{k}, \widehat{h}\right)_{\mathcal{H}^{2}}=0
$$

holds for every $\{\widehat{f}, \widehat{h}\} \in \Gamma$ and hence (3.1) is satisfied. By assumption (G2) one concludes that $\{\widehat{g}, \widehat{k}\} \in \Gamma$, or equivalently, that $\{\widehat{k}, \widehat{g}\} \in \Gamma^{-1}$. This proves the reverse inclusion $\Gamma^{[*]} \subset \Gamma^{-1}$.

Since $\overline{\operatorname{dom}} \Gamma=S^{*}$, the identity $S=\operatorname{ker} \Gamma$ is implied by Proposition 2.3 and (2.15):

$$
\operatorname{ker} \Gamma=(\operatorname{dom} \Gamma)^{[\perp]}=\left(S^{*}\right)^{[\perp]}=S .
$$

This completes the proof.

Note that the boundary relation $\Gamma$ is automatically closed and linear, since it is a unitary relation from the Kreŭ space $\left(\mathfrak{H}^{2}, J_{\mathfrak{H}}\right)$ to the Kreŭn space $\left(\mathcal{H}^{2}, J_{\mathcal{H}}\right)$. However, it can be multi-valued, nondensely defined, or unbounded.

Let $\Gamma$ be a boundary relation for $S^{*}$ and let $T=\operatorname{dom} \Gamma$. According to Proposition 2.12 (see (2.22) ) the linear relation $T$ in $\mathfrak{H}$ satisfies

$$
S \subset T \subset S^{*}, \quad \operatorname{clos} T=S^{*} .
$$

The defect spaces $\mathfrak{N}_{\lambda}(T)$ and $\widehat{\mathfrak{N}}_{\lambda}(T)$ for $T$ are defined as in (2.24). For all elements $\left\{\widehat{f}_{\lambda}, \widehat{h}\right\},\left\{\widehat{g}_{\mu}, \widehat{k}\right\} \in \Gamma$ with $\widehat{f}_{\lambda} \in \widehat{\mathfrak{N}}_{\lambda}(T)$ and $\widehat{g}_{\mu} \in \widehat{\mathfrak{N}}_{\mu}(T)$, one has

$$
(\lambda-\bar{\mu})\left(f_{\lambda}, g_{\mu}\right)_{\mathfrak{H}}=\left(h^{\prime}, k\right)_{\mathcal{H}}-\left(h, k^{\prime}\right)_{\mathcal{H}}, \quad \lambda, \mu \in \mathbb{C} \backslash \mathbb{R},
$$

which follows from the identity (3.1). Hence, the subspace $\widehat{\mathfrak{N}}_{\lambda}(T)$ is positive in the Krĕn space $\left(\mathfrak{H}^{2}, J_{\mathfrak{H}}\right)$ for $\lambda \in \mathbb{C}_{+}$and negative for $\lambda \in \mathbb{C}_{-}$.

Definition 3.3. The Weyl family $M(\cdot)$ of $S$ corresponding to the boundary relation $\Gamma: \mathfrak{H}^{2} \mapsto \mathcal{H}^{2}$ is defined by $M(\lambda):=\Gamma\left(\widehat{\mathfrak{N}}_{\lambda}(T)\right)$, i.e.,

$$
M(\lambda):=\left\{\widehat{h} \in \mathcal{H}^{2}:\left\{\widehat{f}_{\lambda}, \widehat{h}\right\} \in \Gamma \text { for some } \widehat{f_{\lambda}}=\{f, \lambda f\} \in \mathfrak{H}^{2}\right\},
$$

where $\lambda \in \mathbb{C} \backslash \mathbb{R}$. In the case where $M(\cdot)$ is operator-valued it is called the Weyl function of $S$ corresponding to the boundary relation $\Gamma$.

It will be shown that each Weyl family is a Nevanlinna family, and conversely, that each Nevanlinna family can be realized as the Weyl family of a minimal boundary relation.

Definition 3.4. The boundary relation $\Gamma: \mathfrak{H}^{2} \mapsto \mathcal{H}^{2}$ is called minimal if

$$
\mathfrak{H}=\mathfrak{H}_{\text {min }}:=\overline{\operatorname{span}}\left\{\mathfrak{N}_{\lambda}(T): \lambda \in \mathbb{C}_{+} \cup \mathbb{C}_{-}\right\} .
$$


Since $\mathfrak{N}_{\lambda}(T)$ is dense in $\mathfrak{N}_{\lambda}\left(S^{*}\right)$ (cf. Lemma 2.14) the boundary relation $\Gamma$ : $\mathfrak{H}^{2} \mapsto \mathcal{H}^{2}$ is minimal if and only if $S$ is simple. In general, if $S_{\text {min }}$ is the simple part of $S$, the restriction $\Gamma_{\text {min }}: \mathfrak{H}_{\text {min }}^{2} \mapsto \mathcal{H}^{2}$ of the linear relation $\Gamma$ to $\mathfrak{H}_{\text {min }}$ is a boundary relation for $S_{m i n}^{*}$. Clearly, the Weyl families corresponding to the linear relations $\Gamma$ and $\Gamma_{\min }$ coincide.

Associate with $\Gamma$ the following linear relations which are not necessarily closed:

$$
\begin{aligned}
& \Gamma_{0}=\left\{\{\widehat{f}, h\}:\{\widehat{f}, \widehat{h}\} \in \Gamma, \widehat{h}=\left\{h, h^{\prime}\right\}\right\}, \\
& \Gamma_{1}=\left\{\left\{\widehat{f}, h^{\prime}\right\}:\{\widehat{f}, \widehat{h}\} \in \Gamma, \widehat{h}=\left\{h, h^{\prime}\right\}\right\} .
\end{aligned}
$$

It is clear that

$$
\operatorname{dom} M(\lambda)=\Gamma_{0}\left(\widehat{\mathfrak{N}}_{\lambda}(T)\right) \subset \operatorname{ran} \Gamma_{0}, \quad \operatorname{ran} M(\lambda)=\Gamma_{1}\left(\widehat{\mathfrak{N}}_{\lambda}(T)\right) \subset \operatorname{ran} \Gamma_{1} .
$$

If the boundary relation $\Gamma$ is single-valued, the triplet $\left\{\mathcal{H}, \Gamma_{0}, \Gamma_{1}\right\}$ will be called a boundary triplet associated with the boundary relation $\Gamma: \mathfrak{H}^{2} \mapsto \mathcal{H}^{2}$. In this case the Weyl family corresponding to the boundary triplet $\left\{\mathcal{H}, \Gamma_{0}, \Gamma_{1}\right\}$ can also be defined via the equality

$$
\Gamma_{1}\left(\left\{f_{\lambda}, \lambda f_{\lambda}\right\}\right)=M(\lambda) \Gamma_{0}\left(\left\{f_{\lambda}, \lambda f_{\lambda}\right\}\right), \quad\left\{f_{\lambda}, \lambda f_{\lambda}\right\} \in T .
$$

The following fact is also useful. Let $\Gamma: \mathfrak{H}^{2} \mapsto \mathcal{H}^{2}$ be a boundary relation for $S^{*}$. Then

$$
\Gamma^{\top}=\left(\begin{array}{cc}
0 & 1 \\
-1 & 0
\end{array}\right) \Gamma
$$

is a unitary relation from $\mathfrak{H}^{2}$ to $\mathcal{H}^{2}$. Clearly, $\Gamma^{\top}$ is also a boundary relation for $S^{*}$ (so that, in particular, $\operatorname{ker} \Gamma^{\top}=S$ ). Consequently, if $M(\cdot)$ is the Weyl family for $\Gamma$, then $-M(\cdot)^{-1}$ is the Weyl family for $\Gamma^{\top}$.

3.2. Orthogonal coupling associated with a boundary relation. In this subsection the linear transform $\mathcal{J}$ introduced in Subsection 2.4 will be used to obtain some criteria for a linear relation $\Gamma: \mathfrak{H}^{2} \mapsto \mathcal{H}^{2}$ to be a boundary relation. For a boundary relation $\Gamma$ from $\mathfrak{H}^{2}$ to $\mathcal{H}^{2}$ the relation $\widetilde{A}=\mathcal{J}(\Gamma)$ is defined by (2.16). In the following proposition some results of Subsection 2.4 are reformulated in terms of boundary relations.

Proposition 3.5. Let $\Gamma$ be a subspace in $\mathfrak{H}^{2} \times \mathcal{H}^{2}$ and let $S=\operatorname{ker} \Gamma$. Then $\Gamma$ is a boundary relation for $S^{*}$ if and only if $\widetilde{A}=\mathcal{J}(\Gamma)$ is a selfadjoint linear relation in $\mathfrak{H} \oplus \mathcal{H}$. In this case the boundary relation $\Gamma$ is minimal if and only if $\widetilde{A}=\mathcal{J}(\Gamma)$ is a minimal selfadjoint extension of $S_{2}=$ mul $\Gamma$.

Proof. The first statement is immediate from Propositions 2.10, 2.12, and Definition 3.1. By Definition 3.4 the minimality of $\Gamma$ is equivalent to the simplicity of $S$ which, in turn, is equivalent to the minimality of $\widetilde{A}=\mathcal{J}(\Gamma)$ as a selfadjoint extension of $S_{2}$ by Lemma 2.14 .

Proposition 3.6. The linear relation $\Gamma: \mathfrak{H}^{2} \mapsto \mathcal{H}^{2}$ is a boundary relation for $S^{*}$ if and only if the following conditions hold:

(i) $\operatorname{dom} \Gamma$ is dense in $S^{*}$;

(ii) $\Gamma$ is closed and isometric from the Kreĭn space $\left(\mathfrak{H}^{2}, J_{\mathfrak{H}}\right)$ to the Kreĭn space $\left(\mathcal{H}^{2}, J_{\mathcal{H}}\right)$

(iii) $\operatorname{ran}\left(\Gamma\left(\widehat{\mathfrak{N}}_{\lambda}(T)\right)+\lambda\right)=\mathcal{H}$ for some (and, hence, for all) $\lambda \in \mathbb{C}_{+}$and for some (and, hence, for all) $\lambda \in \mathbb{C}_{-}$. 
Proof. Let $\Gamma$ be a boundary relation for $S^{*}$. Then (i) is satisfied by definition. Furthermore, by Proposition [3.2, $\Gamma$ is a unitary relation from the Kreln space $\left(\mathfrak{H}^{2}, J_{\mathfrak{H}}\right)$ to the Kreln space $\left(\mathcal{H}^{2}, J_{\mathcal{H}}\right)$. Hence, (ii) is also satisfied. The transform $\widetilde{A}=\mathcal{J}(\Gamma)$ is selfadjoint, which implies that $\operatorname{ran}(\widetilde{A}-\lambda)=\mathfrak{H} \oplus \mathcal{H}$ for all $\lambda \in \mathbb{C} \backslash \mathbb{R}$. In particular,

$$
\mathfrak{H}_{2}:=\left(\begin{array}{c}
0 \\
\mathcal{H}
\end{array}\right) \subset \operatorname{ran}(\widetilde{A}-\lambda) .
$$

It follows from (2.16) that

$$
\widetilde{A}-\lambda=\left\{\left\{\left(\begin{array}{c}
f \\
h
\end{array}\right),\left(\begin{array}{c}
f^{\prime}-\lambda f \\
-h^{\prime}-\lambda h
\end{array}\right)\right\}:\left\{\left(\begin{array}{c}
f \\
f^{\prime}
\end{array}\right),\left(\begin{array}{c}
h \\
h^{\prime}
\end{array}\right)\right\} \in \Gamma\right\},
$$

which together with (3.5) and (3.8) gives (iii).

Conversely, assume that the linear relation $\Gamma$ satisfies (i), (ii), and (iii). By (ii) and Proposition 2.10 it follows that $\widetilde{A}=\mathcal{J}(\Gamma)$ is closed and symmetric. In order to prove that $\widetilde{A}$ is selfadjoint it suffices to show that $\operatorname{ran}(\widetilde{A}-\lambda)$ is dense for some $\lambda \in \mathbb{C}_{+}$and for some $\lambda \in \mathbb{C}_{-}$. It follows from (iii) and (3.9) that $\mathfrak{H}_{2} \subset \operatorname{ran}(\widetilde{A}-\lambda)$. To complete the argument, assume that $\varphi \in \mathfrak{H}_{1}$ is orthogonal to $\operatorname{ran}(\widetilde{A}-\lambda)$. This implies that $\{\varphi, \bar{\lambda} \varphi\} \in T^{*}$, where $T=\operatorname{dom} \Gamma$. By (i) $T$ is dense in $S^{*}$ and hence $S=T^{*}$ and $\{\varphi, \bar{\lambda} \varphi\} \in S$. Since $S$ is symmetric this yields $\varphi=0$.

Next it will be shown that for every closed symmetric linear relation $S$ there exists a boundary relation for $S^{*}$; in the case of equal defect numbers this fact is well known.

Proposition 3.7. Let $S$ be any closed symmetric linear relation with arbitrary defect numbers in a Hilbert space $\mathfrak{H}$. Then there exists a boundary relation $\Gamma$ : $\mathfrak{H}^{2} \mapsto \mathcal{H}^{2}$ for $S^{*}$.

Proof. Let $\widetilde{A}$ be any selfadjoint exit space extension of $S$ with the property $\widetilde{A} \cap \mathfrak{H}^{2}=$ $S$. Then by Proposition 2.12 the transform $\Gamma=\mathcal{J}^{-1}(\widetilde{A})$ of $\widetilde{A}$ satisfies $\operatorname{ker} \Gamma=S$ and hence by Proposition $3.5 \Gamma$ defines a boundary relation for $S^{*}$. A particular construction of such an extension $\widetilde{A}$ can be given as follows.

In the orthogonal sum $\mathfrak{H} \oplus \mathfrak{H}$ the relation $S \oplus(-S)$ is closed and symmetric with equal defect numbers. Define the relation $\widetilde{A}$ in $\mathfrak{H} \oplus \mathfrak{H}$ by

$$
\widetilde{A}=\left\{\widehat{f}=\left\{\left(\begin{array}{c}
f_{1} \\
f_{2}
\end{array}\right),\left(\begin{array}{c}
f_{1}^{\prime} \\
-f_{2}^{\prime}
\end{array}\right)\right\}: \widehat{f}_{1}, \widehat{f_{2}} \in S^{*}, P_{\mathfrak{N}} \widehat{f}_{1}=P_{\mathfrak{N}} \widehat{f_{2}}\right\},
$$

where $\widehat{f}_{1}=\left\{f_{1}, f_{1}^{\prime}\right\}, \widehat{f}_{2}=\left\{f_{2}, f_{2}^{\prime}\right\}$, and $P_{\mathfrak{N}}$ is the orthogonal projection from $S^{*}$ onto $\mathfrak{N}=\widehat{\mathfrak{N}}_{i}\left(S^{*}\right) \oplus \widehat{\mathfrak{N}}_{-i}\left(S^{*}\right)$; cf. (2.6). The elements $\widehat{f}_{j} \in S^{*}, j=1,2$, in (3.10) have the representations

$$
\left\{f_{j}, f_{j}^{\prime}\right\}=\left\{h_{j}, h_{j}^{\prime}\right\} \widehat{+}\left\{\varphi_{i}, i \varphi_{i}\right\} \widehat{+}\left\{\varphi_{-i},-i \varphi_{-i}\right\},
$$

where $\left\{h_{j}, h_{j}^{\prime}\right\} \in S,\left\{\varphi_{i}, i \varphi_{i}\right\} \in S^{*},\left\{\varphi_{-i},-i \varphi_{-i}\right\} \in S^{*}$. With this notation a typical element of the Cayley transform $U$ of $\widetilde{A}$,

$$
U=\left\{\left\{u^{\prime}-i u, u^{\prime}+i u\right\}:\left\{u, u^{\prime}\right\} \in \widetilde{A}\right\},
$$

is of the form

$$
\left\{\left(\begin{array}{c}
h_{1}^{\prime}-i h_{1}-2 i \varphi_{-i} \\
-\left(h_{2}^{\prime}+i h_{2}\right)-2 i \varphi_{i}
\end{array}\right),\left(\begin{array}{c}
h_{1}^{\prime}+i h_{1}+2 i \varphi_{i} \\
-\left(h_{2}^{\prime}-i h_{2}\right)+2 i \varphi_{-i}
\end{array}\right)\right\} .
$$


This shows immediately that $U$ is isometric and that $\operatorname{dom} U=\operatorname{ran} U=\mathfrak{H} \oplus \mathfrak{H}$, so that $U$ is unitary. Hence $\widetilde{A}$ is a selfadjoint relation which extends $S \oplus(-S)$. Moreover, clearly $S \subset \widetilde{A} \cap \mathfrak{H}^{2}$. In order to prove the reverse inclusion, assume that

$$
\widehat{f}=\left\{\left(\begin{array}{c}
f_{1} \\
f_{2}
\end{array}\right),\left(\begin{array}{c}
f_{1}^{\prime} \\
-f_{2}^{\prime}
\end{array}\right)\right\} \in \widetilde{A} \cap \mathfrak{H}^{2} .
$$

Then $f_{2}=f_{2}^{\prime}=0$, and by the definition (3.10) of the relation $\widetilde{A}$, it follows that $P_{\mathfrak{N}} \widehat{f}_{1}=0$, so that $\left\{f_{1}, f_{1}^{\prime}\right\} \in S$. Hence $\widetilde{A} \cap \mathfrak{H}^{2} \subset S$, and consequently, $\widetilde{A} \cap \mathfrak{H}^{2}=$ $S$.

Remark 3.8. One can simplify the construction of the extension $\widetilde{A}$ in the previous proposition when $S$ is a closed symmetric relation with equal defect numbers. Let $V$ be an isometric mapping from $\mathfrak{N}_{-i}\left(S^{*}\right)$ onto $\mathfrak{N}_{i}\left(S^{*}\right)$ and let $\mathcal{H}=\mathfrak{N}_{i}\left(S^{*}\right)$. Define the linear relation $\widetilde{A}$ by

$$
\widetilde{A}=\left\{\left\{\left(\begin{array}{c}
f \\
\varphi_{i}+V \varphi_{-i}
\end{array}\right),\left(\begin{array}{c}
f^{\prime} \\
-i \varphi_{i}+i V \varphi_{-i}
\end{array}\right)\right\}: \widehat{f}=\left\{f, f^{\prime}\right\} \in S^{*}, \varphi_{ \pm i}=P_{ \pm i} \widehat{f}\right\},
$$

where $P_{ \pm i}$ are the orthoprojections onto $\mathfrak{N}_{ \pm i}\left(S^{*}\right)$ in the decomposition (2.6). Then $\widetilde{A}$ is a selfadjoint extention of $S$ such that $\widetilde{A} \cap \mathfrak{H}^{2}=S$.

The transform $\Gamma=\mathcal{J}^{-1}(\widetilde{A})$ defines a boundary relation for $S^{*}$ with the additional property $\operatorname{ran} \Gamma=\mathcal{H}^{2}$ (so that $\operatorname{dom} \Gamma=S^{*}, \operatorname{mul} \Gamma=\{0,0\}$, which imply that $\Gamma$ is a bounded linear operator); cf. Corollary 2.6. It corresponds to an ordinary boundary triplet $\left\{\mathcal{H}, \Gamma_{0}, \Gamma_{1}\right\}$ with the boundary operators $\Gamma_{0}, \Gamma_{1}$ given by

$$
\Gamma_{0} \widehat{f}=P_{i} \widehat{f}+V P_{-i} \widehat{f}, \quad \Gamma_{1} \widehat{f}=i P_{i} \widehat{f}-i V P_{-i} \widehat{f} ;
$$

cf. 28. For a densely defined operator $S$ with equal defect numbers, the statement of Proposition 3.7 and the formulas (3.11) go back to V.M. Bruck and A.N. Kochuber (see [20]).

\subsection{A characterization of Weyl families: The main realization theorem.}

It was shown in [16] that for every Nevanlinna function $M(\cdot) \in R^{u}[\mathcal{H}]$ there exist a symmetric operator $S$ in a Hilbert space $\mathfrak{H}$ and an ordinary boundary triplet $\left\{\mathcal{H}, \Gamma_{0}, \Gamma_{1}\right\}$ such that the corresponding Weyl function is $M(\cdot)$. Since such a Weyl function is also a $Q$-function of the pair $\left\{S, A_{0}\right\}$ (see [16]), this gives a realization for every uniformly strict Nevanlinna function as a $Q$-function of the pair $\left\{S, A_{0}\right\}$. The latter problem has been originally solved by M.G. Krein and H. Langer in [25] for the case $\overline{\operatorname{dom}} S=\mathfrak{H}$ and extended to the case $\overline{\operatorname{dom}} S \neq \mathfrak{H}$ in [26]. In [16] it was shown that every function $M(\cdot)$ from $R^{s}[\mathcal{H}]$ can be realized as the Weyl function of an appropriate generalized boundary triplet. In this subsection this realization theorem is extended to the class $\widetilde{R}(\mathcal{H})$ of all Nevanlinna families and arbitrary boundary relations. The present approach is based on the generalized Naimark theorem and hence it differs from those used in [25, 26], [16].

Two boundary relations $\Gamma^{(j)}:\left(\mathfrak{H}^{(j)}\right)^{2} \rightarrow \mathcal{H}^{2}, j=1,2$, are said to be unitarily equivalent if there is a unitary operator $U: \mathfrak{H}^{(1)} \rightarrow \mathfrak{H}^{(2)}$ such that

$$
\Gamma^{(2)}=\left\{\left\{\left(\begin{array}{c}
U f \\
U f^{\prime}
\end{array}\right),\left(\begin{array}{c}
h \\
h^{\prime}
\end{array}\right)\right\}:\left\{\left(\begin{array}{c}
f \\
f^{\prime}
\end{array}\right),\left(\begin{array}{c}
h \\
h^{\prime}
\end{array}\right)\right\} \in \Gamma^{(1)}\right\} .
$$

If the boundary relations $\Gamma^{(1)}$ and $\Gamma^{(2)}$ satisfy (3.12) and $S_{j}=\operatorname{ker} \Gamma^{(j)}, T_{j}=$ $\operatorname{dom} \Gamma^{(j)}, j=1,2$, then $S_{2}=U S_{1} U^{-1}$ and $T_{2}=U T_{1} U^{-1}$. 
Theorem 3.9. Let $\Gamma: \mathfrak{H}^{2} \rightarrow \mathcal{H}^{2}$ be a boundary relation for $S^{*}$. Then the corresponding Weyl family $M(\cdot)$ belongs to the class $\widetilde{R}(\mathcal{H})$.

Conversely, if $M(\cdot)$ belongs to the class $\widetilde{R}(\mathcal{H}$ ), then there exists (up to unitary equivalence) a unique minimal boundary relation whose Weyl function coincides with $M(\cdot)$.

Proof. Necessity. If $\Gamma: \mathfrak{H}^{2} \rightarrow \mathcal{H}^{2}$ is a boundary relation, so that $\widetilde{A}$ is selfadjoint, it follows from the (2.16), (3.9) that

$$
(\widetilde{A}-\lambda)^{-1}\left(\begin{array}{c}
f^{\prime}-\lambda f \\
-h^{\prime}-\lambda h
\end{array}\right)=\left(\begin{array}{l}
f \\
h
\end{array}\right), \quad\left\{\left(\begin{array}{c}
f \\
f^{\prime}
\end{array}\right),\left(\begin{array}{c}
h \\
h^{\prime}
\end{array}\right)\right\} \in \Gamma,
$$

which implies

$$
R(\lambda):=P_{\mathcal{H}}(\widetilde{A}-\lambda)^{-1} \uparrow \mathcal{H}=-(M(\lambda)+\lambda)^{-1}, \quad \lambda \in \mathbb{C} \backslash \mathbb{R} .
$$

The latter equality can be rewritten as

$$
M(\lambda)=\{\{R(\lambda) h,-(I+\lambda R(\lambda)) h\}: h \in \mathcal{H}\} .
$$

Since the kernel

$$
K(\lambda, \mu)=\frac{R(\lambda)-R(\mu)^{*}}{\lambda-\bar{\mu}}-R(\mu)^{*} R(\lambda)=\left.P_{\mathcal{H}}(\widetilde{A}-\bar{\mu})^{-1}\left(I-P_{\mathcal{H}}\right)(\widetilde{A}-\lambda)^{-1}\right|_{\mathcal{H}}
$$

is nonnegative (see [36]), it follows that $M(\cdot)$ is a Nevanlinna family. Indeed, for every

one obtains

$$
\left\{f, f^{\prime}\right\}=\{R(\lambda) h,-(I+\lambda R(\lambda)) h\} \in M(\lambda), \quad h \in \mathcal{H},
$$

$$
\frac{\left(f^{\prime}, f\right)-\left(f, f^{\prime}\right)}{\lambda-\bar{\lambda}}=(K(\lambda, \lambda) h, h) \geq 0 .
$$

Sufficiency. Assume that $M(\cdot)$ belongs to $\widetilde{R}(\mathcal{H})$. Then $M_{1}(\lambda):=-(M(\lambda)+\lambda)^{-1}$ belongs to $R[\mathcal{H}]$, and therefore it admits an integral representation of the form (2.29). The estimate $\left\|M_{1}(i y)\right\| \leq 1 / y$ and the monotonicity of $y \operatorname{Im} M_{1}(i y)$ show that the strong limit $s-\lim _{y \rightarrow \infty} y \operatorname{Im} M_{1}(i y)$ exists and defines a bounded operator in $\mathcal{H}$ such that

$$
0 \leq s-\lim _{y \rightarrow \infty} y \operatorname{Im} M_{1}(i y) \leq I_{\mathcal{H}} .
$$

Moreover, $s-\lim _{y \rightarrow \infty} M_{1}(i y)=0$. Hence, the integral representation of $M_{1}(\lambda)$ takes the form

$$
M_{1}(\lambda)=\int_{\mathbb{R}} \frac{d \Sigma(t)}{t-\lambda}, \quad 0 \leq \int_{\mathbb{R}} d \Sigma(t)=s-\lim _{y \rightarrow \infty} y \operatorname{Im} M_{1}(i y) \leq I_{\mathcal{H}} .
$$

Without loss of generality one may assume that $\Sigma(-\infty)=0$, in which case $0 \leq$ $\Sigma(+\infty):=s-\lim _{t \rightarrow \infty} \Sigma(t) \leq I_{\mathcal{H}}$. It follows from the generalized Naimark theorem (cf. [1, 30, 36]) that there is an orthogonal dilation $E(t)$ of $\Sigma(t)$ (i.e. a spectral family of a selfadjoint linear relation $\widetilde{A}$ in some Hilbert space $\widetilde{\mathfrak{H}} \supset \mathcal{H}$ with $\Sigma(t)=$ $\left.P_{\mathcal{H}} E(t)\right|_{\mathcal{H}}$ for all $\left.t \in \mathbb{R}\right)$. Note that $E(\infty)$ is an orthogonal projection in $\widetilde{\mathfrak{H}}$, which is equal to $I_{\widetilde{\mathcal{H}}}$ if and only if $\widetilde{A}$ is an operator. The linear relation $\widetilde{A}$ can be chosen minimal in the sense that

$$
\widetilde{\mathfrak{H}}=\overline{\operatorname{span}}\{\mathcal{H}, E(t) \mathcal{H}: t \in \mathbb{R}\},
$$

which, of course, is equivalent to the minimality of the selfadjoint extension $\widetilde{A}$ with respect to $\mathcal{H}$. Observe, that if $\widetilde{A}$ is minimal, then $\widetilde{A}$ is an operator if and only if $\Sigma(+\infty)=I_{\mathcal{H}}$. 
It follows from (3.14) that $M_{1}(\lambda)$ takes the form

$$
M_{1}(\lambda)=P_{\mathcal{H}}(\widetilde{A}-\lambda)^{-1} \uparrow_{\mathcal{H}}, \quad \lambda \in \mathbb{C} \backslash \mathbb{R},
$$

where $\widetilde{A}$ is a minimal selfadjoint relation with the above properties. Let $\mathfrak{H}=\widetilde{\mathfrak{H}} \ominus \mathcal{H}$. Decompose the graph of $\widetilde{A}$ as in (2.16) according to the decomposition $\widetilde{\mathfrak{H}}=\mathfrak{H} \oplus \mathcal{H}$ and let $\Gamma=\mathcal{J}^{-1}(\widetilde{A})$. Due to Proposition $3.5 \Gamma$ defines a boundary relation for $S^{*}$ where $S:=\operatorname{ker} \Gamma=\widetilde{A} \cap(\widetilde{\mathfrak{H}} \ominus \mathcal{H})^{2}$. By Lemma $2.14 \Gamma$ is minimal by the minimality of the selfadjoint extension $\widetilde{A}$ with respect to $\mathcal{H}$; see (3.15). Now the first part of the present proof shows that the Weyl family associated with $\Gamma$ satisfies (3.13) with the compressed resolvent of $\widetilde{A}$ in $\mathcal{H}$ given by (3.16). Therefore, the Weyl family associated with $\Gamma$ coincides with the given family $M(\cdot) \in \widetilde{R}(\mathcal{H})$.

Uniqueness. Assume that $\Gamma^{(j)}:\left(\mathfrak{H}^{(j)}\right)^{2} \rightarrow \mathcal{H}^{2}, j=1,2$, are two minimal boundary relations with the same Weyl family $M(\lambda)$. Then $\widetilde{A}^{(j)}=\mathcal{J}\left(\Gamma^{(j)}\right), j=1,2$, are two selfadjoint linear relations in Hilbert spaces $\widetilde{\mathfrak{H}}^{(j)}(\supset \mathcal{H})$ minimal with respect to $\mathcal{H}$ and such that

$$
P_{\mathcal{H}}\left(\widetilde{A}^{(j)}-\lambda\right)^{-1} \uparrow \mathcal{H}=-(M(\lambda)+\lambda)^{-1}, \quad \lambda \in \mathbb{C} \backslash \mathbb{R} .
$$

Then the corresponding resolutions of identities $E^{(j)}(t)$ also have the minimality properties

$$
\widetilde{\mathfrak{H}}^{(j)}=\operatorname{clos} \mathcal{L}^{(j)}, \quad \mathcal{L}^{(j)}=\operatorname{span}\left\{\mathcal{H}, E^{(j)}(t) \mathcal{H}: t \in \mathbb{R}\right\},
$$

and by the Stieltjes inversion formula they satisfy the equality $\left.P_{\mathcal{H}} E^{(1)}(t)\right|_{\mathcal{H}}=$ $P_{\mathcal{H}} E^{(2)}(t)\left\lceil_{\mathcal{H}}\right.$ for all $t \in \mathbb{R}$. Define the mapping $U_{0}: \mathcal{L}^{(1)} \rightarrow \mathcal{L}^{(2)}$ by the equalities

$$
U_{0} h=h, \quad U_{0} E^{(1)}(t) h=E^{(2)}(t) h, \quad h \in \mathcal{H}, \quad t \in \mathbb{R} .
$$

It follows from

$$
\begin{aligned}
\left\|E^{(1)}(t) h\right\|_{\tilde{\mathfrak{H}}^{(1)}}^{2} & =\int_{(-\infty, t]} d\left(E^{(1)}(s) h, h\right)_{\tilde{\mathfrak{H}}^{(1)}} \\
& =\int_{(-\infty, t]} d\left(E^{(2)}(s) h, h\right)_{\widetilde{\mathfrak{H}}^{(2)}}=\left\|E^{(2)}(t) h\right\|_{\widetilde{\mathfrak{H}}^{(2)}}^{2}, \quad h \in \mathcal{H},
\end{aligned}
$$

that $U_{0}$ is a well-defined isometric mapping from $\mathcal{L}^{(1)}$ onto $\mathcal{L}^{(2)}$. Its closure $\widetilde{U}$ is a unitary operator from $\widetilde{\mathfrak{H}}^{(1)}$ onto $\widetilde{\mathfrak{H}}^{(2)}$, and according to the decompositions $\widetilde{\mathfrak{H}}^{(j)}=\mathfrak{H}^{(j)} \oplus \mathcal{H}$ it can be represented as $\widetilde{U}=U \oplus I_{\mathcal{H}}$, where $U: \mathfrak{H}^{(1)} \rightarrow \mathfrak{H}^{(2)}$ is unitary. It follows from (3.17) that $\widetilde{U} E_{1}(t)=E_{2}(t) \widetilde{U}$ for all $t \in \mathbb{R}$ and, therefore, the selfadjoint linear relations $\widetilde{A}^{(j)}$ are unitary equivalent:

$$
\widetilde{A}^{(2)}=\left\{\left\{\left(\begin{array}{c}
U f \\
h
\end{array}\right),\left(\begin{array}{c}
U f^{\prime} \\
-h^{\prime}
\end{array}\right)\right\}:\left\{\left(\begin{array}{l}
f \\
h
\end{array}\right),\left(\begin{array}{c}
f^{\prime} \\
-h^{\prime}
\end{array}\right)\right\} \in \widetilde{A}^{(1)}\right\} .
$$

This leads to the unitary equivalence (3.12) of the boundary relations $\Gamma^{(1)}$ and $\Gamma^{(2)}$.

\section{WEYL FAMILIES OF SYMMETRIC OPERATORS}

4.1. Subclasses of Weyl families. The main theorem in the previous section gives a one-to-one correspondence between Nevanlinna families and boundary relations. In this subsection geometric characterizations of subclasses of Nevanlinna families or functions are given in terms of the boundary relation. The following preliminary result is important. 
Lemma 4.1. Let $\Gamma: \mathfrak{H}^{2} \rightarrow \mathcal{H}^{2}$ be a boundary relation for $S^{*}$ with the Weyl family $M(\lambda)=\Gamma\left(\widehat{\mathfrak{N}}_{\lambda}(T)\right)$. Then the following equalities hold for every $\lambda \in \mathbb{C} \backslash \mathbb{R}$ :

(i) $M(\lambda) \cap M(\lambda)^{*}=\operatorname{mul} \Gamma$ and $\operatorname{clos}\left(M(\lambda) \hat{+} M(\lambda)^{*}\right)=\overline{\operatorname{ran}} \Gamma$;

(ii) $\operatorname{ker} M(\lambda) \times\{0\}=\operatorname{mul} \Gamma \cap(\mathcal{H} \times\{0\})$ and $\{0\} \times \operatorname{mul} M(\lambda)=\operatorname{mul} \Gamma \cap(\{0\} \times \mathcal{H})$;

(iii) $\operatorname{ker}\left(M(\lambda)-M(\lambda)^{*}\right)=\operatorname{mul} \Gamma_{0}$ and $\operatorname{ker}\left(M(\lambda)^{-1}-M(\lambda)^{-*}\right)=\operatorname{mul} \Gamma_{1}$.

Proof. (i) Let $\{0, \widehat{h}\} \in \Gamma, \widehat{h}=\left\{h, h^{\prime}\right\}$. Then (3.1) and (3.4) show that for every $\lambda \in \mathbb{C} \backslash \mathbb{R}$ and $\left\{k, k^{\prime}\right\} \in \Gamma\left(\widehat{\mathfrak{N}}_{\lambda}(T)\right)$ the identity $\left(h^{\prime}, k\right)-\left(h, k^{\prime}\right)=0$ holds. Hence, $\widehat{h} \in M(\lambda)^{*}$ for all $\lambda \in \mathbb{C} \backslash \mathbb{R}$, which proves that mul $\Gamma \subset M(\lambda) \cap M(\lambda)^{*}, \lambda \in \mathbb{C} \backslash \mathbb{R}$. Conversely, if $\widehat{h} \in M(\lambda) \cap M(\lambda)^{*}, \lambda \in \mathbb{C} \backslash \mathbb{R}$, then $\left\{\widehat{f_{\lambda}}, \widehat{h}\right\} \in \Gamma$ for some $\widehat{f}_{\lambda} \in \widehat{\mathfrak{N}}_{\lambda}(T)$ and, moreover, according to (3.4) $(\lambda-\bar{\lambda})\left\|f_{\lambda}\right\|^{2}=0$, which implies that $\widehat{f}_{\lambda}=\{0,0\}$. Therefore, $\{0, \widehat{h}\} \in \Gamma$, and this proves the reverse inclusion $M(\lambda) \cap M(\lambda)^{*} \subset \operatorname{mul} \Gamma$, $\lambda \in \mathbb{C} \backslash \mathbb{R}$. Hence, the first statement of (i) has been shown. The second statement in (i) follows from the first one by taking adjoints and applying Proposition 2.12.

(ii) Let $\widehat{h}=\{h, 0\} \in \operatorname{mul} \Gamma, h \in \mathcal{H}$. Then $\widehat{h} \in M(\lambda)$ for all $\lambda \in \mathbb{C} \backslash \mathbb{R}$, so that $h \in \operatorname{ker} M(\lambda), \lambda \in \mathbb{C} \backslash \mathbb{R}$. Conversely, let $h \in \operatorname{ker} M(\lambda)$, so that $\widehat{h}=\{h, 0\} \in M(\lambda)$ for some $\lambda \in \mathbb{C} \backslash \mathbb{R}$. Then $\left\{\widehat{f_{\lambda}}, \widehat{h}\right\} \in \Gamma$ for some $\widehat{f}_{\lambda}=\left\{f_{\lambda}, \lambda f_{\lambda}\right\} \in \widehat{\mathfrak{N}}_{\lambda}(T)$ and (3.4) gives $(\lambda-\bar{\lambda})\left\|f_{\lambda}\right\|^{2}=(0, h)-(h, 0)=0$. Hence, $f_{\lambda}=0$ and $\widehat{f}_{\lambda}=0$, which shows that $\widehat{h} \in \operatorname{mul} \Gamma \cap(\mathcal{H} \times\{0\})$. This proves the first equality in (i). The proof of the second equality is similar.

(iii) These identities follow immediately from (i) and (2.1), and the definition in (3.6).

Remark 4.2. Lemma4.1 combined with the realization theorem proved in the previous section (see Theorem 3.9) yields immediately the following invariance results for an arbitrary Nevanlinna family $M(\cdot) \in \widetilde{R}(\mathcal{H})$ :

$$
\begin{aligned}
& M(\lambda) \cap M(\lambda)^{*}, \\
& \operatorname{ker} M(\lambda), \quad \operatorname{mul} M(\lambda), \\
& \operatorname{ker}\left(M(\lambda)-M(\lambda)^{*}\right), \quad \operatorname{ker}\left(M(\lambda)^{-1}-M(\lambda)^{-*}\right)
\end{aligned}
$$

do not depend on $\lambda \in \mathbb{C} \backslash \mathbb{R}$.

This indicates that via Theorem 3.9 boundary relations in fact offer a new method for studying function and spectral theoretical properties of Nevanlinna families by means of geometric properties of boundary relations, and vice versa. Observe that direct function theoretical proofs for the invariance properties of Nevanlinna families formulated in (4.1) may be based e.g. on an application of the maximality principle.

Lemma 4.1 gives also the following result.

Corollary 4.3. Let $\Gamma: \mathfrak{H}^{2} \rightarrow \mathcal{H}^{2}$ be a boundary relation for $S^{*}$ with the Weyl family $M(\lambda)=\Gamma\left(\widehat{\mathfrak{N}}_{\lambda}(T)\right)$. Then the following equalities hold for every $\lambda \in \mathbb{C} \backslash \mathbb{R}$ :

$$
\overline{\operatorname{dom}} M(\lambda)=\overline{\operatorname{ran}} \Gamma_{0}, \quad \overline{\operatorname{ran}} M(\lambda)=\overline{\operatorname{ran}} \Gamma_{1} .
$$

Proof. The definition of the Weyl family $M(\lambda)$, the symmetry property $M(\lambda)^{*}=$ $M(\bar{\lambda})$, and part (i) of Lemma 4.1 imply that

$$
M(\lambda) \widehat{+} M(\bar{\lambda}) \subset \operatorname{ran} \Gamma \subset \operatorname{clos}(M(\lambda) \widehat{+} M(\bar{\lambda})), \quad \lambda \in \mathbb{C} \backslash \mathbb{R} .
$$


Since mul $M(\lambda)$ is independent from $\lambda \in \mathbb{C} \backslash \mathbb{R}$, the equality $\overline{\operatorname{dom}} M(\lambda)=\overline{\operatorname{dom}} M(\bar{\lambda})$ holds. Now the identities (4.2) follow from (4.3).

In general, the first inclusion in (4.3) need not be an equality; see Examples 6.5 and 6.6. However, sufficient conditions for the equality to hold can be found in the next lemma.

Lemma 4.4. Let $\Gamma: \mathfrak{H}^{2} \rightarrow \mathcal{H}^{2}$ be a boundary relation for $S^{*}$ with the Weyl family $M(\lambda)=\Gamma\left(\widehat{\mathfrak{N}}_{\lambda}(T)\right)$. Then the following statements are equivalent:

(i) $\operatorname{ran} \Gamma$ is closed;

(ii) $M(\lambda) \widehat{+} M(\lambda)^{*}$ is closed for some (or, equivalently, for every) $\lambda \in \mathbb{C} \backslash \mathbb{R}$;

(iii) $T=S^{*}$.

If any one of these conditions is satisfied, then

$$
M(\lambda) \widehat{+} M(\lambda)^{*}=\operatorname{ran} \Gamma, \quad \lambda \in \mathbb{C} \backslash \mathbb{R} .
$$

Proof. (i) $\Leftrightarrow$ (iii) This is clear from Proposition 2.3 since $T=\operatorname{dom} \Gamma$ dense in $S^{*}$.

(ii) $\Rightarrow$ (i) If (ii) holds for some $\lambda \in \mathbb{C} \backslash \mathbb{R}$, then part (i) of Lemma 4.1] and (4.3) yield

$$
\operatorname{ran} \Gamma \subset \overline{\operatorname{ran}} \Gamma=M(\lambda) \widehat{+} M(\lambda)^{*} \subset \operatorname{ran} \Gamma .
$$

(iii) $\Rightarrow$ (ii) Von Neumann's formula (2.5) implies that for every $\lambda \in \mathbb{C} \backslash \mathbb{R}$

$$
\Gamma\left(\widehat{\mathfrak{N}}_{\lambda}(T)\right) \hat{+} \Gamma\left(\widehat{\mathfrak{N}}_{\bar{\lambda}}(T)\right)=\operatorname{ran} \Gamma,
$$

which is closed since ran $\Gamma$ is closed by the equivalence of (i) and (iii).

If one of the conditions (i), (ii), or (iii) is satisfied, then the identity (4.4) is clear from the above arguments.

Proposition 4.5. Let $\Gamma: \mathfrak{H}^{2} \rightarrow \mathcal{H}^{2}$ be a boundary relation for $S^{*}$ with the Weyl family $M(\lambda)=\Gamma\left(\widehat{\mathfrak{N}}_{\lambda}(T)\right)$. Then:

(i) $M(\cdot) \in R(\mathcal{H})$ if and only if mul $\Gamma \cap(\{0\} \times \mathcal{H})=\{0\}$;

(ii) $M(\cdot) \in R^{s}(\mathcal{H})$ if and only if $\operatorname{ran} \Gamma$ is dense in $\mathcal{H}^{2}$;

(iii) $M(\cdot) \in R^{u}(\mathcal{H})$ if and only if $\operatorname{ran} \Gamma=\mathcal{H}^{2}$.

Proof. (i) Observe that $M(\cdot) \in R(\mathcal{H})$ if and only if mul $M(\lambda)=\{0\}$. Hence, the statement follows from part (ii) of Lemma 4.1.

(ii) By definition $M(\cdot) \in R^{s}(\mathcal{H})$ if and only if $M(\lambda) \cap M(\lambda)^{*}=\{0\}$. The statement now follows from part (i) of Lemma 4.1 and Proposition 2.3.

(iii) By definition $M(\cdot) \in R^{u}(\mathcal{H})$ if and only if $M(\lambda) \widehat{+} M(\lambda)^{*}=\mathcal{H}^{2}$. Hence, if $M(\cdot) \in R^{u}(\mathcal{H})$, then clearly $\operatorname{ran} \Gamma=\mathcal{H}^{2}$; cf. the inclusion (4.3). Conversely, if $\operatorname{ran} \Gamma=\mathcal{H}^{2}$, then $\operatorname{ran} \Gamma$ is closed, so that by Lemmas 4.1 and 4.4, $M(\lambda) \widehat{+} M(\lambda)^{*}=$ $\operatorname{ran} \Gamma=\mathcal{H}^{2}$, and thus $M(\cdot) \in R^{u}(\mathcal{H})$.

The class $\widetilde{R}_{\text {inv }}(\mathcal{H})$ is the set of all $M(\cdot) \in \widetilde{R}(\mathcal{H})$ such that $\Gamma_{0}\left(\widehat{\mathfrak{N}}_{\lambda}(T)\right)=\mathcal{H}_{0}$ for some linear subspace $\mathcal{H}_{0} \subset \mathcal{H}$ with $\operatorname{clos} \mathcal{H}_{0}=(\operatorname{mul} M(\lambda))^{\perp}$.

Corollary 4.6. The invariant subclasses $R_{\text {inv }}(\mathcal{H})$ and $R_{\text {inv }}^{s}(\mathcal{H})$ are characterized by:

(i) $M(\cdot) \in R_{\text {inv }}(\mathcal{H})$ if and only if mul $\Gamma \cap(\{0\} \times \mathcal{H})=\{0\}$ and $\Gamma_{0}\left(\widehat{\mathfrak{N}}_{\lambda}(T)\right)=\mathcal{H}_{0}$ for some dense linear subspace $\mathcal{H}_{0} \subset \mathcal{H}$;

(ii) $M(\cdot) \in R_{\text {inv }}^{s}(\mathcal{H})$ if and only if mul $\Gamma=\{0\}$ and $\Gamma_{0}\left(\widehat{\mathfrak{N}}_{\lambda}(T)\right)=\mathcal{H}_{0}$ for some dense linear subspace $\mathcal{H}_{0} \subset \mathcal{H}$. 
Proposition 4.7. Let $\Gamma: \mathfrak{H}^{2} \rightarrow \mathcal{H}^{2}$ be a boundary relation for $S^{*}$ with the Weyl family $M(\lambda)=\Gamma\left(\widehat{\mathfrak{N}}_{\lambda}(T)\right)$. Then:

(i) $M(\cdot) \in \widetilde{R}[\mathcal{H}]$ if and only if $\Gamma_{0}\left(\widehat{\mathfrak{N}}_{\lambda}(T)\right)=\mathcal{H}_{0}, \lambda \in \mathbb{C} \backslash \mathbb{R}$, where $\mathcal{H}_{0} \subset \mathcal{H}$ is a closed linear subspace;

(ii) $M(\cdot) \in R[\mathcal{H}]$ if and only if $\Gamma_{0}\left(\widehat{\mathfrak{N}}_{\lambda}(T)\right)=\mathcal{H}, \lambda \in \mathbb{C} \backslash \mathbb{R}$;

(iii) $M(\cdot) \in R^{s}[\mathcal{H}]$ if and only if mul $\Gamma_{0}=\{0\}$ and $\Gamma_{0}\left(\widehat{\mathfrak{N}}_{\lambda}(T)\right)=\mathcal{H}, \lambda \in \mathbb{C} \backslash \mathbb{R}$.

Proof. For any $M(\cdot) \in \widetilde{R}(\mathcal{H})$ the following orthogonal decomposition holds:

$$
\mathcal{H}=\overline{\operatorname{dom}} M(\lambda) \oplus \operatorname{mul} M(\lambda),
$$

and here mul $M(\lambda)$ does not depend on $\lambda \in \mathbb{C} \backslash \mathbb{R}$. Define $\mathcal{H}_{0}=\mathcal{H} \ominus$ mul $M(\lambda)$. Since $\Gamma_{0}\left(\widehat{\mathfrak{N}}_{\lambda}(T)\right)=\operatorname{dom} M(\lambda)$, parts (i) and (ii) follow from the definitions of $\widetilde{R}[\mathcal{H}]$ and $R[\mathcal{H}]$.

For (iii) observe that $M(\cdot) \in R^{s}[\mathcal{H}]$ if and only if $M(\cdot) \in R[\mathcal{H}]$ and $\operatorname{ker} \operatorname{Im} M(\lambda)=$ $\{0\}$. Hence, the assertion follows from Lemma 4.1 .

4.2. Boundary relations and their $\gamma$-fields. The identity (3.4) shows that

$$
\operatorname{ker}\left(\Gamma_{0}\left\lceil\widehat{\mathfrak{N}}_{\lambda}(T)\right)=\{0\}, \quad \operatorname{ker}\left(\Gamma_{1}\left\lceil\widehat{\mathfrak{N}}_{\lambda}(T)\right)=\{0\}, \quad \operatorname{ker}\left(\Gamma\left\lceil\widehat{\mathfrak{N}}_{\lambda}(T)\right)=\{0\},\right.\right.\right.
$$

for all $\lambda \in \mathbb{C} \backslash \mathbb{R}$. In particular, the inverse of $\Gamma_{0} \vdash \widehat{\mathfrak{N}}_{\lambda}(T)$ is a single-valued linear mapping from $\Gamma_{0}\left(\widehat{\mathfrak{N}}_{\lambda}(T)\right)=\operatorname{dom} M(\lambda)$ onto $\widehat{\mathfrak{N}}_{\lambda}(T)$; it is denoted by $\widehat{\gamma}(\lambda):=$ $\left(\Gamma_{0}\left\lceil\widehat{\mathfrak{N}}_{\lambda}(T)\right)^{-1}\right.$. The $\gamma$-field $\gamma(\cdot)$ associated with the boundary relation $\Gamma: \mathfrak{H}^{2} \rightarrow \mathcal{H}^{2}$ is defined by

$$
\gamma(\lambda)=\left\{\left\{h, f_{\lambda}\right\}:\left\{\widehat{f_{\lambda}}, \widehat{h}\right\} \in \Gamma, \widehat{f_{\lambda}}=\left\{f_{\lambda}, \lambda f_{\lambda}\right\} \in T, \widehat{h}=\left\{h, h^{\prime}\right\} \in \mathcal{H}^{2}\right\},
$$

for $\lambda \in \mathbb{C} \backslash \mathbb{R}$, so that $\gamma(\lambda)$ corresponds to the first component of the mapping $\widehat{\gamma}(\lambda)$. It maps $\Gamma_{0}\left(\widehat{\mathfrak{N}}_{\lambda}(T)\right)$ onto $\mathfrak{N}_{\lambda}(T)$ and satisfies $\gamma(\lambda) \Gamma_{0} \widehat{f}_{\lambda}=f_{\lambda}$ for all $\widehat{f}_{\lambda} \in \widehat{\mathfrak{N}}_{\lambda}(T)$. With $\gamma(\lambda)$ the relation $\Gamma \mid \widehat{\mathfrak{N}}_{\lambda}(T)$ can be rewritten as follows:

$$
\Gamma \nmid \widehat{\mathfrak{N}}_{\lambda}(T):=\left\{\left\{\{\gamma(\lambda) h, \lambda \gamma(\lambda) h\},\left\{h, h^{\prime}\right\}\right\}:\left\{h, h^{\prime}\right\} \in M(\lambda)\right\}, \quad \lambda \in \mathbb{C} \backslash \mathbb{R} .
$$

In the case that $\Gamma$ is single-valued one can decompose $\Gamma=\Gamma_{0} \oplus \Gamma_{1}$. Then by part (ii) of Lemma 4.1 the corresponding Weyl family $M(\cdot)$ is operator-valued. In this case the identity (4.6) takes the form

$$
\Gamma_{0} \widehat{\gamma}(\lambda) h=h, \quad \Gamma_{1} \widehat{\gamma}(\lambda) h=M(\lambda) h, \quad h \in \operatorname{dom} M(\lambda), \quad \lambda \in \mathbb{C} \backslash \mathbb{R} .
$$

These formulas are typically used in the case of ordinary boundary triplets for defining the corresponding Weyl function.

Proposition 4.8. Let $\Gamma: \mathfrak{H}^{2} \rightarrow \mathcal{H}^{2}$ be a boundary relation for $S^{*}$ with the Weyl family $M(\lambda)=\Gamma\left(\widehat{\mathfrak{N}}_{\lambda}(T)\right)$ and let $\widetilde{A}=\mathcal{J}(\Gamma)$ be as in (2.16). Then the corresponding $\gamma$-field $\gamma(\cdot)$ in (4.5) and the Weyl family $M(\cdot)$ are connected by

$$
(\widetilde{A}-\lambda)^{-1}\left(\begin{array}{l}
0 \\
\varphi
\end{array}\right)=-\left(\begin{array}{c}
\gamma(\lambda)(M(\lambda)+\lambda)^{-1} \varphi \\
(M(\lambda)+\lambda)^{-1} \varphi
\end{array}\right), \quad \varphi \in \mathcal{H}, \quad \lambda \in \mathbb{C} \backslash \mathbb{R} .
$$

Furthermore, the $\gamma$-field $\gamma(\cdot)$ satisfies with $h \in \operatorname{dom} M(\lambda), k \in \operatorname{dom} M(\mu)$, and $\lambda, \mu \in \mathbb{C} \backslash \mathbb{R}$ the identity

$$
\frac{\left(M_{s}(\lambda) h, k\right)_{\mathcal{H}}-\left(h, M_{s}(\mu) k\right)_{\mathcal{H}}}{\lambda-\bar{\mu}}=(\gamma(\lambda) h, \gamma(\mu) k)_{\mathfrak{H}},
$$


and, in particular,

$$
\operatorname{ker} \gamma(\lambda)=\operatorname{mul} \Gamma_{0}=\operatorname{ker}\left(M(\lambda)-M(\lambda)^{*}\right) .
$$

Proof. It follows from (2.16) and (4.5) that

$$
(\widetilde{A}-\lambda)^{-1}\left(\begin{array}{c}
0 \\
-h^{\prime}-\lambda h
\end{array}\right)=\left(\begin{array}{c}
\gamma(\lambda) h \\
h
\end{array}\right), \quad h \in \Gamma_{0}\left(\widehat{\mathfrak{N}}_{\lambda}(T)\right),
$$

which gives (4.7) immediately. The identity (4.8) follows from (3.4) and the description (4.6). Finally, the identities in (4.9) are obtained from the definition (4.5) and Lemma 4.1 .

The identity (4.7) shows the sense in which the mapping $\gamma(\lambda): \Gamma_{0}\left(\widehat{\mathfrak{N}}_{\lambda}(T)\right) \rightarrow$ $\mathfrak{N}_{\lambda}(T)$ can be seen to be holomorphic. In general, the closure of the mapping $\gamma(\lambda)$ is not single-valued; cf. Example 6.7. However, there is a useful sufficient condition for $\gamma(\lambda)$ to have a single-valued closure.

Proposition 4.9. Assume that for some $\lambda \in \mathbb{C} \backslash \mathbb{R}$ the inclusion

$$
\Gamma_{0}\left(\widehat{\mathfrak{N}}_{\lambda}(T)\right) \subset \Gamma_{0}\left(\widehat{\mathfrak{N}}_{\bar{\lambda}}(T)\right)
$$

is satisfied. Then $\gamma(\lambda)$ admits a single-valued closure.

Proof. It follows from (4.8) that for all $h \in \operatorname{dom} M(\lambda)$

$$
(\lambda-\bar{\lambda})(\gamma(\lambda) h, \gamma(\lambda) h)_{\mathfrak{H}}=\left(M_{s}(\lambda) h, h\right)_{\mathcal{H}}-\left(h, M_{s}(\lambda) h\right)_{\mathcal{H}},
$$

and the assumption $\operatorname{dom} M(\lambda) \subset \operatorname{dom} M(\lambda)^{*}$ then implies that

$$
(\gamma(\lambda) h, \gamma(\lambda) h)_{\mathfrak{H}}=\left(\frac{M_{s}(\lambda)-M_{s}(\lambda)^{*}}{\lambda-\bar{\lambda}} h, h\right)_{\mathcal{H}} .
$$

Now, for each $\lambda \in \mathbb{C} \backslash \mathbb{R}$, the operator

$$
N(\lambda):=\frac{M_{s}(\lambda)-M_{s}(\lambda)^{*}}{\lambda-\bar{\lambda}}
$$

is a nonnegative densely defined operator in $\mathcal{H} \ominus$ mul $M(\lambda)$. Therefore, both quadratic forms in (4.11) are closable (see 22]). Hence, $\gamma(\lambda)$ admits a single-valued closure.

It follows from the identity $(N(\lambda) h, h)=(\gamma(\lambda) h, \gamma(\lambda) h), h \in \operatorname{dom} M(\lambda)$, that the operator $N(\lambda)$ is nonnegative. Hence, it has a Friedrichs extension $N_{F}(\lambda)$. According to the Second Representation Theorem (see [22]) the single-valued closure of $\gamma(\lambda)$, denoted by $\gamma^{* *}(\lambda)$, satisfies $\operatorname{dom} \gamma^{* *}(\lambda)=\operatorname{dom} N_{F}(\lambda)^{1 / 2}$. Observe that the original mapping is onto $\mathfrak{N}_{\lambda}(T)$ and its closure is into $\mathfrak{N}_{\lambda}\left(S^{*}\right)$. In general the closure does not map onto $\mathfrak{N}_{\lambda}\left(S^{*}\right)$; cf. Example 6.8. If (4.10) is not satisfied the closure of $\gamma(\lambda)$ can be multi-valued; see Example 6.7.

4.3. Characterization of domain invariance. The boundary relation for $S^{*}$ and the associated mappings $\Gamma_{0}$ and $\Gamma_{1}$ in (3.6) induce two linear relations:

$$
A_{0}=\operatorname{ker} \Gamma_{0}, \quad A_{1}=\operatorname{ker} \Gamma_{1},
$$

in the Hilbert space $\mathfrak{H}$. Clearly, these relations are symmetric and satisfy

$$
S \subset A_{0} \subset T, \quad S \subset A_{1} \subset T .
$$

The relations $A_{0}$ and $A_{1}$ need not be closed, and their defect numbers may be unequal; cf. Example 6.3. The following lemma is useful in the further study of these relations. 
Lemma 4.10. Let $\Gamma: \mathcal{H}^{2} \rightarrow \mathfrak{H}^{2}$ be a boundary relation for $S^{*}$. Then for $j=1,2$ :

(i) $\Gamma_{j}\left(\widehat{\mathfrak{N}}_{\lambda}(T)\right) \subset \operatorname{ran} \Gamma_{j} \subset \operatorname{clos}\left(\Gamma_{j}\left(\widehat{\mathfrak{N}}_{\lambda}(T)\right)\right)$ for all $\lambda \in \mathbb{C} \backslash \mathbb{R}$;

(ii) $\operatorname{ran} \Gamma_{j}^{[*]}=\operatorname{ker} \Gamma_{j}$, where $\Gamma_{j}$ is understood as a linear subspace of $\mathfrak{H}^{2} \times \mathcal{H}^{2}$;

(iii) if $\operatorname{ran} \Gamma_{j}$ is closed, then $A_{j}$ is closed.

The condition in (iii) is satisfied if $\Gamma_{j}\left(\widehat{\mathfrak{N}}_{\lambda}(T)\right)$ is closed for some $\lambda \in \mathbb{C} \backslash \mathbb{R}$.

Proof. (i) The first inclusion is obvious. Since by definition $\Gamma_{0}\left(\widehat{\mathfrak{N}}_{\lambda}(T)\right)=\operatorname{dom} M(\lambda)$ and $\Gamma_{1}\left(\widehat{\mathfrak{N}}_{\lambda}(T)\right)=\operatorname{ran} M(\lambda)$, the second inclusion is immediate from Corollary 4.3 ,

(ii) Identifying $\Gamma_{0}$ as a linear subspace of $\Gamma \subset \mathfrak{H}^{2} \times \mathcal{H}^{2}$, it takes the form

$$
\Gamma_{0}=\left\{\{\widehat{f},\{h, 0\}\}:\left\{\widehat{f},\left\{h, h^{\prime}\right\}\right\} \in \Gamma\right\} .
$$

Assume that $\widehat{g} \in \operatorname{ran} \Gamma_{0}^{[*]}$, i.e. $\{\widehat{k}, \widehat{g}\} \in \Gamma_{0}^{[*]}$ for some $\widehat{k}=\left\{k, k^{\prime}\right\}$. Then for all $\{\widehat{f},\{h, 0\}\} \in \Gamma_{0}$,

$$
0=\left(J_{\mathfrak{H}} \widehat{g}, \widehat{f}\right)_{\mathfrak{H}^{2}}-\left(J_{\mathcal{H}}\left\{k, k^{\prime}\right\},\{h, 0\}\right)_{\mathcal{H}^{2}}=\left(J_{\mathfrak{H}} \widehat{g}, \widehat{f}\right)_{\mathfrak{H}^{2}}-\left(J_{\mathcal{H}}\left\{0, k^{\prime}\right\},\left\{h, h^{\prime}\right\}\right)_{\mathcal{H}^{2}} .
$$

This means that $\left\{\left\{0, k^{\prime}\right\}, \widehat{g}\right\} \in \Gamma^{[*]}=\Gamma^{-1}$ or equivalently that $\left\{\widehat{g},\left\{0, k^{\prime}\right\}\right\} \in \Gamma$, i.e., $\widehat{g} \in A_{0}=\operatorname{ker} \Gamma_{0}$. Therefore, $\operatorname{ran} \Gamma_{0}^{[*]}=\operatorname{ker} \Gamma_{0}$. Similarly one proves the identity $\operatorname{ran} \Gamma_{1}^{[*]}=\operatorname{ker} \Gamma_{1}$.

(iii) Let $\operatorname{ran} \Gamma_{j}$ be closed, so that also $\operatorname{ran} \Gamma_{j}^{* *}$ is closed. By Proposition 2.2, or, more precisely, by its Kreĭn space version, it follows that $\operatorname{ran} \Gamma_{j}^{[*]}$ is closed. The equalities in (ii) now imply that $A_{j}$ is closed.

The last statement is clear from the inclusions in (i).

The condition in (iii) is sufficient, but not necessary. In fact, in Example 6.6 $\operatorname{ran} \Gamma_{0}$ is not closed, while $A_{0}$ is selfadjoint.

Proposition 4.11. The following statements are equivalent:

(i) $\mathcal{H}_{0}:=\Gamma_{0}\left(\widehat{\mathfrak{N}}_{\lambda}(T)\right)$ is independent from $\lambda \in \mathbb{C}_{+}$(resp. from $\lambda \in \mathbb{C}_{-}$);

(ii) $\mathfrak{N}_{\mu}(T) \subset \operatorname{ran}\left(A_{0}-\lambda\right)$ for all $\lambda, \mu \in \mathbb{C}_{+}$(resp. for all $\lambda, \mu \in \mathbb{C}_{-}$), $\lambda \neq \mu$.

If one of these conditions is satisfied, then the $\gamma$-field $\gamma(\cdot)$ satisfies

$$
\gamma(\lambda)=\left[I+(\lambda-\mu)\left(A_{0}-\lambda\right)^{-1}\right] \gamma(\mu), \quad \lambda, \mu \in \mathbb{C}_{+}\left(\mathbb{C}_{-}\right) .
$$

Proof. (i) $\Rightarrow$ (ii) Let $\mathcal{H}_{0}=\Gamma_{0}\left(\widehat{\mathfrak{N}}_{\lambda}(T)\right)$ for all $\lambda \in \mathbb{C}_{+}$. It follows from (4.6) that for every $h \in \mathcal{H}_{0}$ there exist $h^{\prime}, h^{\prime \prime} \in \mathcal{H}$ such that

$$
\begin{aligned}
& \left\{\{\gamma(\lambda) h, \lambda \gamma(\lambda) h\},\left\{h, h^{\prime}\right\}\right\} \in \Gamma \mid \widehat{\mathfrak{N}}_{\lambda}(T) \subset \Gamma, \\
& \left\{\{\gamma(\mu) h, \mu \gamma(\mu) h\},\left\{h, h^{\prime \prime}\right\}\right\} \in \Gamma \mid \widehat{\mathfrak{N}}_{\mu}(T) \subset \Gamma .
\end{aligned}
$$

Hence,

and therefore

$$
\left\{\{(\gamma(\lambda)-\gamma(\mu)) h,(\lambda \gamma(\lambda)-\mu \gamma(\mu)) h\},\left\{0, h^{\prime}-h^{\prime \prime}\right\}\right\} \in \Gamma,
$$

$$
\{(\gamma(\lambda)-\gamma(\mu)) h,(\lambda \gamma(\lambda)-\mu \gamma(\mu)) h\} \in A_{0} .
$$

It follows from (4.13) that

$$
\{(\gamma(\lambda)-\gamma(\mu)) h,(\lambda-\mu) \gamma(\mu) h\} \in A_{0}-\lambda
$$

so that $\gamma(\mu) h \in \operatorname{ran}\left(A_{0}-\lambda\right)$ for every $h \in \mathcal{H}_{0}$. Therefore (ii) follows.

(ii) $\Rightarrow$ (i) Let $h \in \Gamma_{0}\left(\widehat{\mathfrak{N}}_{\mu}(T)\right)$. By definition there is an element $h^{\prime} \in \mathcal{H}$ so that

$$
\left\{\{\gamma(\mu) h, \mu \gamma(\mu) h\},\left\{h, h^{\prime}\right\}\right\} \in \Gamma .
$$


The assumption in (ii) shows that $\gamma(\mu) h \in \operatorname{ran}\left(A_{0}-\lambda\right)$, so that there is an element $k \in \mathfrak{H}$ such that $\{k, \gamma(\mu) h+\lambda k\} \in A_{0}$. Hence, there exists $\varphi \in \mathcal{H}$ such that

$$
\{\{(\lambda-\mu) k,(\lambda-\mu) \gamma(\mu) h+\lambda(\lambda-\mu) k\},\{0, \varphi\}\} \in \Gamma .
$$

It follows from (4.14) and 4.15) that

$$
\left\{\{\gamma(\mu) h+(\lambda-\mu) k, \lambda(\gamma(\mu) h+(\lambda-\mu) k)\},\left\{h, h^{\prime}+\varphi\right\}\right\} \in \Gamma .
$$

In other words, $h \in \Gamma_{0}\left(\widehat{\mathfrak{N}}_{\lambda}(T)\right)$. Hence $\Gamma_{0}\left(\widehat{\mathfrak{N}}_{\mu}(T)\right) \subset \Gamma_{0}\left(\widehat{\mathfrak{N}}_{\lambda}(T)\right)$, and equality follows by symmetry.

Now assume that one of the equivalent conditions (i) or (ii) is satisfied. The assumption (i) implies the identity (4.13), which may be rewritten as

$$
\{\gamma(\mu) h, \gamma(\lambda) h\} \in I+(\lambda-\mu)(A-\lambda)^{-1},
$$

where $\left(A_{0}-\lambda\right)^{-1}$ is bounded on $\operatorname{ran}\left(A_{0}-\lambda\right)$, since $A_{0}$ is symmetric. Hence, (4.12) holds.

Corollary 4.12. If (i) or, equivalently, (ii) in Proposition 4.11 holds for all $\lambda, \mu \in$ $\mathbb{C} \backslash \mathbb{R}$, then $A_{0}$ is essentially selfadjoint and (4.12) holds for all $\lambda, \mu \in \mathbb{C} \backslash \mathbb{R}$.

Proof. Assume that $S$ is simple. Then part (ii) of Proposition 4.11 implies that $\overline{\operatorname{ran}}\left(A_{0}-\lambda\right)=\mathfrak{H}, \lambda \in \mathbb{C} \backslash \mathbb{R}$, so that $A_{0}$ is essentially selfadjoint.

If $S$ is not simple, decompose $S=S^{\prime} \oplus S^{\prime \prime}$, where $S^{\prime \prime}$ is selfadjoint. The symmetric extension $A_{0}$ of $S$ decomposes accordingly: $A_{0}=A_{0}^{\prime} \oplus S^{\prime \prime}$. Hence, the earlier argument shows that $A_{0}^{\prime}$ is essentially selfadjoint, so that $A_{0}$ itself is also essentially selfadjoint.

Now, the case of equality in the first inclusion of (i) in Lemma 4.10 is characterized.

Theorem 4.13. For every fixed $\lambda \in \mathbb{C}_{+}\left(\mathbb{C}_{-}\right)$the following statements are equivalent:

(i) $\operatorname{ran}\left(A_{0}-\lambda\right)=\mathfrak{H}$ (i.e. $A_{0}$ is maximal symmetric);

(ii) $T=A_{0} \widehat{+} \widehat{\mathfrak{N}}_{\lambda}(T)$;

(iii) $\Gamma_{0}\left(\widehat{\mathfrak{N}}_{\lambda}(T)\right)=\operatorname{ran} \Gamma_{0}$.

If (i), (ii), or (iii) holds for some $\lambda \in \mathbb{C}_{+}\left(\mathbb{C}_{-}\right)$, then (i), (ii), and (iii) hold for every $\lambda \in \mathbb{C}_{+}\left(\mathbb{C}_{-}\right)$and, moreover, $S$ satisfies

$$
S=\left\{\{f, g\} \in A_{0}^{*}:(g-\bar{\lambda} f, \gamma(\lambda) h)_{\mathfrak{H}}=0, \quad h \in \mathcal{H}_{0}:=\Gamma_{0}\left(\widehat{\mathfrak{N}}_{\lambda}(T)\right)\right\},
$$

where $\lambda \in \mathbb{C}_{+}\left(\mathbb{C}_{-}\right)$.

Proof. (i) $\Rightarrow$ (ii) If $\operatorname{ran}\left(A_{0}-\lambda\right)=\mathfrak{H}$ for $\lambda \in \mathbb{C}_{+}$, it follows that

$$
S^{*}=A_{0} \widehat{+} \widehat{\mathfrak{N}}_{\lambda}\left(S^{*}\right) \text {. }
$$

Now observe that $T \subset S^{*}, A_{0} \subset T$, and $T \cap \widehat{\mathfrak{N}}_{\lambda}\left(S^{*}\right)=\widehat{\mathfrak{N}}_{\lambda}(T)$, which gives (ii).

(ii) $\Rightarrow$ (i) The identity in (ii) shows that

$$
\operatorname{ran}(T-\lambda)=\operatorname{ran}\left(A_{0}-\lambda\right), \quad \lambda \in \mathbb{C}_{+} .
$$

Since $\widetilde{A}$ is selfadjoint, it follows from (2.16) and (2.21) that $\operatorname{ran}(T-\lambda)=\mathfrak{H}$ for every $\lambda \in \mathbb{C} \backslash \mathbb{R}$. Hence, in particular $\operatorname{ran}\left(A_{0}-\lambda\right)=\mathfrak{H}$ for $\lambda \in \mathbb{C}_{+}$, which gives (i). 
(ii) $\Rightarrow$ (iii) Let $h \in \operatorname{ran} \Gamma_{0}$, so that $\left\{\widehat{f},\left\{h, h^{\prime}\right\}\right\} \in \Gamma$ for some $h^{\prime} \in \mathcal{H}, \widehat{f} \in T$. Decompose $\widehat{f}=\widehat{f}_{0}+\widehat{f}_{\lambda}$, where $\left\{\widehat{f}_{0},\{0, k\}\right\} \in \Gamma$ and $\left\{\widehat{f}_{\lambda},\left\{h, h^{\prime}-k\right\}\right\} \in \widehat{\mathfrak{N}}_{\lambda}(T)$. This shows that $h \in \Gamma_{0}\left(\widehat{\mathfrak{N}}_{\lambda}(T)\right)$, and (iii) follows.

(iii) $\Rightarrow$ (ii) Since clearly $A_{0} \widehat{+} \widehat{\mathfrak{N}}_{\lambda}(T) \subset T$, it suffices to prove the reverse inclusion. Let $\widehat{f} \in T$, so that $\left\{\widehat{f},\left\{h, h^{\prime}\right\}\right\} \in \Gamma$ for some $h, h^{\prime} \in \mathcal{H}$. According to (iii) there exists $\widehat{f}_{\lambda} \in \widehat{\mathfrak{N}}_{\lambda}(T)$, such that $\left\{\widehat{f}_{\lambda}, h\right\} \in \Gamma_{0}$, i.e., $\left\{\widehat{f}_{\lambda},\left\{h, h^{\prime \prime}\right\}\right\} \in \Gamma$ for some $h^{\prime \prime} \in \mathcal{H}$. This implies that $\left\{\widehat{f}-\widehat{f}_{\lambda},\left\{0, h^{\prime}-h^{\prime \prime}\right\}\right\} \in \Gamma$, and therefore $\widehat{f}_{0}:=\widehat{f}-\widehat{f}_{\lambda} \in A_{0}$, which shows that $\widehat{f}=\widehat{f}_{0}+\widehat{f}_{\lambda} \in A_{0} \widehat{+} \widehat{\mathfrak{N}}_{\lambda}(T)$. Hence, (ii) follows.

If any of the equivalent statements (i), (ii), or (iii) holds for some $\lambda \in \mathbb{C}_{+}\left(\mathbb{C}_{-}\right)$, then symmetry of $A_{0}$ forces that these statements hold for every $\lambda \in \mathbb{C}_{+}\left(\mathbb{C}_{-}\right)$. Moreover, it follows from (ii) that

$$
T^{*}=A_{0}^{*} \cap\left(\widehat{\mathfrak{N}}_{\lambda}(T)\right)^{*},
$$

which leads to the identity (4.16).

As a consequence of Theorem 4.13 one obtains criteria for $A_{0}$ to be selfadjoint.

Corollary 4.14. The relation $A_{0}$ is selfadjoint if and only if one (and hence all) of the statements (i), (ii), or (iii) in Theorem 4.13 holds for some $\lambda \in \mathbb{C}_{+}$and for some $\lambda \in \mathbb{C}_{-}$. Moreover, in this case

$$
S=\left\{\{f, g\} \in A_{0}:(g-\bar{\lambda} f, \gamma(\lambda) h)_{\mathfrak{H}}=0, h \in \mathcal{H}_{0}:=\Gamma_{0}\left(\widehat{\mathfrak{N}}_{\lambda}(T)\right)\right\}, \quad \lambda \in \mathbb{C} \backslash \mathbb{R}
$$

Next, the case of equality in the second inclusion of (i) in Lemma 4.10 is characterized.

Proposition 4.15. For every fixed $\lambda \in \mathbb{C}_{+}\left(\mathbb{C}_{-}\right)$the following statements are equivalent:

(i) $\Gamma_{0}\left(\widehat{\mathfrak{N}}_{\lambda}(T)\right)$ is closed;

(ii) $\overline{\operatorname{ran}}\left(A_{0}-\lambda\right)=\mathfrak{H}$, and $\operatorname{ran} \Gamma_{0}$ is closed.

In this case $A_{0}$ is closed and $\operatorname{ran}\left(A_{0}-\lambda\right)=\mathfrak{H}$ for every $\lambda \in \mathbb{C}_{+}\left(\mathbb{C}_{-}\right)$, so that $A_{0}$ is maximal symmetric.

Proof. (i) $\Rightarrow$ (ii) Let $\Gamma_{0}\left(\widehat{\mathfrak{N}}_{\lambda}(T)\right)$ be closed for some $\lambda \in \mathbb{C}_{+}\left(\mathbb{C}_{-}\right)$. Then it follows from Lemma 4.10 that ran $\Gamma_{0}$ is closed, and Theorem 4.13 implies that $\operatorname{ran}\left(A_{0}-\lambda\right)=$ $\mathfrak{H}$ for $\lambda \in \mathbb{C}_{+}\left(\mathbb{C}_{-}\right)$.

(ii) $\Rightarrow$ (i) If ran $\Gamma_{0}$ is closed, then $A_{0}$ is closed by Lemma 4.10, Therefore the assumption $\overline{\operatorname{ran}}\left(A_{0}-\lambda\right)=\mathfrak{H}$ for $\lambda \in \mathbb{C}_{+}\left(\mathbb{C}_{-}\right)$leads to $\operatorname{ran}\left(A_{0}-\lambda\right)=\mathfrak{H}, \lambda \in$ $\mathbb{C}_{+}\left(\mathbb{C}_{-}\right)$. Then by Theorem $4.13 \operatorname{ran} \Gamma_{0}=\Gamma_{0}\left(\widehat{\mathfrak{N}}_{\lambda}(T)\right)$, and this subspace is closed by the assumptions in (ii).

The last statement follows from Lemma 4.10.

As a consequence of Proposition 4.15 one obtains some further invariance results known for an arbitrary Nevanlinna family $M(\cdot) \in \widetilde{R}(\mathcal{H})$. This in turn leads to a more precise statement concerning $A_{0}$ in the previous proposition.

Proposition 4.16. Let the Nevanlinna family $M(\cdot) \in \widetilde{R}(\mathcal{H})$ be the Weyl family associated to the boundary relation $\Gamma: \mathfrak{H}^{2} \rightarrow \mathcal{H}^{2}$ via Theorem 3.9 , Then:

(i) if $\operatorname{dom} M\left(\lambda_{0}\right)\left(\operatorname{ran} M\left(\lambda_{0}\right)\right)$ is closed for some $\lambda_{0} \in \mathbb{C} \backslash \mathbb{R}$, then $\operatorname{ran} \Gamma_{0}$ (resp. ran $\left.\Gamma_{1}\right)$ is closed, and the operator part of $M(\lambda)\left(\right.$ of $\left.M(\lambda)^{-1}\right)$ is bounded for every $\lambda \in \mathbb{C} \backslash \mathbb{R}$; 
(ii) if $\operatorname{ran} \Gamma_{0}\left(\operatorname{ran} \Gamma_{1}\right)$ is not closed, then $\operatorname{dom} M(\lambda)$ (resp. $\operatorname{ran} M(\lambda)$ ) is not closed, and the operator part of $M(\lambda)\left(\right.$ of $\left.M(\lambda)^{-1}\right)$ is unbounded for every $\lambda \in \mathbb{C} \backslash \mathbb{R}$.

Proof. It is sufficient to prove (i). Since $M\left(\lambda_{0}\right)^{*}=M\left(\bar{\lambda}_{0}\right)$, it follows from Proposition 2.2 that $\operatorname{dom} M\left(\lambda_{0}\right)$ is closed if and only if $\operatorname{dom} M\left(\bar{\lambda}_{0}\right)$ is closed. In this case $\operatorname{dom} M\left(\lambda_{0}\right)=\operatorname{dom} M\left(\bar{\lambda}_{0}\right)=\operatorname{ran} \Gamma_{0}$ by Corollary 4.3. Now it is clear that the properties in part (ii) of Proposition 4.15 are satisfied for every $\lambda \in \mathbb{C} \backslash \mathbb{R}$. Hence dom $M(\lambda)=\Gamma_{0}\left(\widehat{\mathfrak{N}}_{\lambda}(T)\right)$ is closed for every $\lambda \in \mathbb{C} \backslash \mathbb{R}$. By the closed graph theorem this means that the operator part of $M(\lambda), \lambda \in \mathbb{C} \backslash \mathbb{R}$, is bounded (see (2.27)). Similarly one proves the assertion for $\operatorname{ran} M(\lambda)$.

Corollary 4.17. If one of the equivalent statements (i) or (ii) in Proposition 4.15 holds at a single point $\lambda_{0} \in \mathbb{C} \backslash \mathbb{R}$, then $A_{0}$ is selfadjoint.

Proof. The equalities $\operatorname{dom} M\left(\lambda_{0}\right)=\operatorname{dom} M\left(\bar{\lambda}_{0}\right)=\operatorname{ran} \Gamma_{0}$ were shown in the proof of Proposition 4.16, and consequently the equality $\operatorname{ran}\left(A_{0}-\lambda\right)=\mathfrak{H}$ holds in fact for every $\lambda \in \mathbb{C} \backslash \mathbb{R}$.

Further invariance results concerning the spectra of an arbitrary Nevanlinna family $M(\cdot) \in \widetilde{R}(\mathcal{H})$ are now easily established.

Proposition 4.18. Let $M(\cdot) \in \widetilde{R}(\mathcal{H})$ be a Nevanlinna family, let $\alpha \in \mathbb{R}$, and let $\lambda_{0} \in \mathbb{C} \backslash \mathbb{R}$. Then:

(i) if $M\left(\lambda_{0}\right) \in[\mathcal{H}]$, then $M(\lambda) \in[\mathcal{H}]$ for all $\lambda \in \mathbb{C} \backslash \mathbb{R}$;

(ii) if $\alpha \in \rho\left(M\left(\lambda_{0}\right)\right)$, then $\alpha \in \rho(M(\lambda))$ for all $\lambda \in \mathbb{C} \backslash \mathbb{R}$;

(iii) if $\alpha \in \sigma_{p}\left(M\left(\lambda_{0}\right)\right)$, then $\alpha \in \sigma_{p}(M(\lambda))$ for all $\lambda \in \mathbb{C} \backslash \mathbb{R}$;

(iv) if $\alpha \in \sigma_{c}\left(M\left(\lambda_{0}\right)\right)$, then $\alpha \in \sigma_{c}(M(\lambda))$ for all $\lambda \in \mathbb{C} \backslash \mathbb{R}$.

Proof. To prove the statements let $M(\cdot) \in \widetilde{R}(\mathcal{H})$ be the Weyl family associated to the boundary relation $\Gamma: \mathfrak{H}^{2} \rightarrow \mathcal{H}^{2}$ via Theorem 3.9

(i) By Lemma $4.1 \overline{\operatorname{dom}} M(\lambda)=(\text { mul } M(\lambda))^{\perp}$ does not depend on $\lambda \in \mathbb{C} \backslash \mathbb{R}$, and hence the statement follows from part (i) of Proposition 4.16

(ii) The condition $\alpha \in \rho(M(\lambda))$ means that $\left(M(\lambda)-\alpha I_{\mathcal{H}}\right)^{-1} \in[\mathcal{H}]$. Since $-\left(M(\lambda)-\alpha I_{\mathcal{H}}\right)^{-1} \in \widetilde{R}(\mathcal{H})$, the assertion is obtained immediately from (i).

(iii) For every $\alpha \in \mathbb{R}$ is clear that $M(\lambda)-\alpha I_{\mathcal{H}} \in \widetilde{R}(\mathcal{H})$. Therefore, by Lemma 4.1 $\operatorname{ker}\left(M(\lambda)-\alpha I_{\mathcal{H}}\right)$ does not depend on $\lambda \in \mathbb{C} \backslash \mathbb{R}$.

(iv) Observe that $\sigma(M(\lambda))=\sigma_{p}(M(\lambda)) \cup \sigma_{c}(M(\lambda))$. Hence the statement follows by combining (ii) and (iii).

Remark 4.19. It follows from the proof of Proposition 4.18 that the eigenspaces $\operatorname{ker}\left(M(\lambda)-\alpha I_{\mathcal{H}}\right), \alpha \in \mathbb{R}$, actually do not depend on $\lambda \in \mathbb{C} \backslash \mathbb{R}$.

According to Corollary 4.14 the assumption that $A_{0}$ is selfadjoint is equivalent to the decomposition $T=A_{0} \hat{+} \widehat{\mathfrak{N}}_{\lambda}(T), \lambda \in \mathbb{C} \backslash \mathbb{R}$. Clearly, this decomposition is direct: $\widehat{\mathfrak{N}}_{\lambda}(T) \cap A_{0}=\{0,0\}$. In this case the intersection $\mathfrak{N}_{\lambda}(T) \cap \operatorname{dom} A_{0}$ can be described as follows.

Proposition 4.20. Assume that $A_{0}$ is selfadjoint. Then for $\lambda \in \mathbb{C} \backslash \mathbb{R}$,

$$
\mathfrak{N}_{\lambda}(T) \cap \operatorname{dom} A_{0}=\left(A_{0}-\lambda\right)^{-1}(\operatorname{mul} T) .
$$

Moreover, $h \in \Gamma_{0}\{0,-\omega\}, \omega \in \operatorname{mul} T$, if and only if $\gamma(\lambda) h=\left(A_{0}-\lambda\right)^{-1} \omega$ for $\lambda \in \mathbb{C} \backslash \mathbb{R}$. 
Proof. The identity in (4.17) is equivalent to

$$
\begin{aligned}
\{\{f, \lambda f\} \in T: f & \left.\in \operatorname{dom} A_{0}\right\} \\
& =\left\{\left\{\left(A_{0}-\lambda\right)^{-1} \omega, \lambda\left(A_{0}-\lambda\right)^{-1} \omega\right\}: \omega \in \operatorname{mul} T\right\} .
\end{aligned}
$$

Let $\{f, \lambda f\} \in T$ have the property that $f \in \operatorname{dom} A_{0}$. Then there is an element $\omega \in \mathfrak{H}$ for which $f=\left(A_{0}-\lambda\right)^{-1} \omega$. Hence

$$
\{f, \lambda f\}-\left\{\left(A_{0}-\lambda\right)^{-1} \omega,\left(I+\lambda\left(A_{0}-\lambda\right)^{-1}\right) \omega\right\}=\{0,-\omega\} .
$$

Since the elements in the left-hand side belong to $T$, it follows that $\omega \in \operatorname{mul} T$. Hence the left-hand side of (4.18) is contained in the right-hand side.

Conversely, observe that for $\omega \in \mathfrak{H}$

$$
\left\{\left(A_{0}-\lambda\right)^{-1} \omega, \lambda\left(A_{0}-\lambda\right)^{-1} \omega\right\}=\left\{\left(A_{0}-\lambda\right)^{-1} \omega,\left(I+\lambda\left(A_{0}-\lambda\right)^{-1}\right) \omega\right\} \widehat{+}\{0,-\omega\} .
$$

Thus, if $\omega \in \operatorname{mul} T$, then the elements in the right-hand side belong to the relation $A_{0} \widehat{+}(\{0\} \times \operatorname{mul} T) \subset T$. Hence the right-hand side of (4.18) is contained in the left-hand side.

It follows from (4.19) that $h \in \Gamma_{0}\{0,-\omega\}$ if and only if $h \in \Gamma_{0}\{f, \lambda f\}$, and, by definition of $\gamma(\lambda)$, this is equivalent to $f=\gamma(\lambda) h$.

Remark 4.21. The assumption that $A_{0}$ is selfadjoint is essential for the inclusion $\operatorname{mul} T \subset \operatorname{ran}\left(A_{0}-\lambda\right)$. If $A_{0}$ is not selfadjoint, the identity (4.17) need not be valid; cf. Example 6.5.

The next result is a strengthening of Lemma 4.10.

Proposition 4.22. Let $\Gamma$ be a boundary relation for $S^{*}$ and let $\operatorname{ran} \Gamma_{0}$ be closed. Then:

(i) $\Gamma\left(A_{0}\right)$ is essentially selfadjoint in $\mathcal{H}$, and the defect numbers of $S$ are equal;

(ii) if $A_{0}^{*} \subset \operatorname{dom} \Gamma$, then $A_{0}=\operatorname{ker} \Gamma_{0}$ is selfadjoint and the linear relation $\Gamma_{0}$ is closed;

(iii) if $\operatorname{ran} \Gamma$ is closed, and in particular if the defect numbers of $S$ are finite, then $A_{0}=\operatorname{ker} \Gamma_{0}$ is selfadjoint and the linear relation $\Gamma_{0}$ is closed.

Proof. (i) By assumption $\operatorname{dom} T_{2}=\operatorname{ran} \Gamma_{0}$ is closed. Then also $\operatorname{dom}\left(\operatorname{clos} T_{2}\right)=$ $\operatorname{dom} S_{2}^{*}$ is closed, and hence by Proposition $2.2 \operatorname{dom} S_{2}$ is closed. Decompose $S_{2}=$ $S_{0} \oplus\left(\{0\} \times \operatorname{mul} S_{2}\right)$, where $S_{0}$ is the operator part of $S_{2}$ in $\mathcal{H} \ominus\left(\operatorname{mul} S_{2}\right)=\overline{\operatorname{dom}} S_{2}^{*}=$ $\operatorname{dom} T_{2}\left(\supset \operatorname{dom} S_{2}\right)$ and $\{0\} \times \operatorname{mul} S_{2}$ is a selfadjoint relation in mul $S_{2}$. Since $\operatorname{dom} S_{0}=\operatorname{dom} S_{2}$ is closed, $S_{0}$ is a bounded symmetric operator in dom $T_{2}$. It is well known that $S_{0}$ has bounded selfadjoint extensions in $\operatorname{dom} T_{2}$; cf. e.g. 11. Let $B_{0}$ be a bounded selfadjoint extension of $S_{0}$ in $\operatorname{dom} T_{2}$. Then $S_{0}^{*}=B_{0} \widehat{+}\left(\{0\} \times \operatorname{mul} S_{0}^{*}\right)$, since clearly $\left(B_{0} \widehat{+}\left(\{0\} \times \operatorname{mul} S_{0}^{*}\right)\right)^{*}=S_{0}$ and, moreover, $B_{0} \widehat{+}\left(\{0\} \times \operatorname{mul} S_{0}^{*}\right)$ is closed, which follows from the fact that $B_{0}$ is a closed bounded operator in $\operatorname{dom} T_{2}$. Consequently,

$$
\operatorname{clos} T_{2}=S_{2}^{*}=S_{0}^{*} \oplus\left(\{0\} \times \operatorname{mul} S_{2}\right)=B_{0} \widehat{+}\left(\{0\} \times \operatorname{mul} S_{2}^{*}\right) .
$$

To prove essential selfadjointness of $\Gamma\left(A_{0}\right)$ first observe that

$$
\Gamma\left(A_{0}\right)=\operatorname{mul} \Gamma \widehat{+}\left(\{0\} \times \operatorname{mul} T_{2}\right)=S_{2} \widehat{+}\left(\{0\} \times \operatorname{mul} T_{2}\right) .
$$

Since $A_{0}$ is symmetric, $\Gamma\left(A_{0}\right)$ is symmetric by Proposition 2.13 Moreover, mul $T$ is dense in mul $S_{2}^{*}$, which follows from (4.20) and the boundedness of $B_{0}$. Since 
$\operatorname{dom} S_{2}=\operatorname{dom} S_{0}$ is closed, this together with (4.20) implies that

$$
\begin{aligned}
\Gamma\left(A_{0}\right)^{*} & =S_{2}^{*} \cap\left(\left(\operatorname{mul} T_{2}\right)^{\perp} \times \mathcal{H}\right)=S_{2}^{*} \cap\left(\operatorname{dom} S_{2} \times \mathcal{H}\right) \\
& =S_{0} \widehat{+}\left(\{0\} \times \operatorname{mul} S_{2}^{*}\right) \subset \operatorname{clos} \Gamma\left(A_{0}\right) \subset \Gamma\left(A_{0}\right)^{*} .
\end{aligned}
$$

Hence, $\Gamma\left(A_{0}\right)$ is essentially selfadjoint, and the defect numbers of $S_{2}$ and $S=S_{1}$ are equal.

(ii) By Proposition $2.13 A_{0}$ is essentially selfadjoint, and by Lemma 4.10 it is closed. Thus, $A_{0}$ is selfadjoint. The closedness of $\Gamma_{0}$ follows from the general implication

$$
A_{0}=A_{0}^{*}, \quad \operatorname{ran} \Gamma_{0} \text { is closed } \Rightarrow \quad \Gamma_{0} \text { is closed. }
$$

(iii) If $\operatorname{ran} \Gamma$ is closed, then equivalently $T=\operatorname{dom} \Gamma$ is closed, i.e., $T=S^{*}$. Consequently, $A_{0}^{*} \subset \operatorname{dom} \Gamma$ and the statement is obtained from part (ii). Observe that if the defect numbers of $S$ are finite, then $T$ is closed as a finite-dimensional extension of $S$.

Remark 4.23. Proposition 4.22 gives rise to the following observations:

(i) mul $M(\lambda)=\operatorname{mul} S_{2}$, and the operator part $M_{s}(\lambda)$ of $M(\lambda)$ acts on $\overline{\operatorname{dom}} T_{2}$;

(ii) if $\operatorname{ran} \Gamma_{0}$ is closed, then $\Gamma\left(A_{0}\right)$ is selfadjoint if and only if mul $T_{2}$ is closed;

(iii) if $\operatorname{ran} \Gamma_{1}$ is closed, then $\Gamma\left(A_{1}\right)$ is selfadjoint if and only if $\operatorname{ker} T_{2}$ is closed;

(iv) if $\operatorname{ran} \Gamma_{0}$ and $\operatorname{ran} \Gamma_{1}$ both are closed and $\Gamma\left(A_{0}\right)$ or $\Gamma\left(A_{1}\right)$ is selfadjoint, then $A_{0}$ and $A_{1}$ both are selfadjoint. Moreover, $A_{0} \widehat{+} A_{1}=T$, so that $A_{0}$ and $A_{1}$ are disjoint with respect to $S$;

(v) part (iii) of Proposition 4.22 applies in particular to the case $\operatorname{ran} \Gamma=\mathcal{H}^{2}$. This implies for instance the equality $R^{u}(\mathcal{H})=R^{u}[\mathcal{H}]$; cf. Theorem 4.13 .

4.4. Domain invariance and operator representations of Weyl families. A Weyl family $M(\cdot) \in \widetilde{R}(\mathcal{H})$ belongs to the subclass $\widetilde{R}_{i n v}(\mathcal{H})$ when there exists a linear (not necessarily closed) subspace $\mathcal{H}_{0} \subset \mathcal{H}$ such that

$$
\operatorname{dom} M(\lambda)=\Gamma_{0}\left(\widehat{\mathfrak{N}}_{\lambda}(T)\right)=\mathcal{H}_{0}, \quad \lambda \in \mathbb{C} \backslash \mathbb{R} .
$$

In this case Proposition 4.9 may be applied to show that the closure of the $\gamma$-field $\gamma(\cdot)$ is single-valued. Note that in this proposition the notations $\left[\mathcal{H}_{0}, \mathfrak{N}_{\lambda}(T)\right]$ and $\left[\mathcal{H}_{0}\right]$ refer to the bounded linear operators in the respective spaces, even when $\mathcal{H}_{0}$ is not complete.

Proposition 4.24. Let $\Gamma: \mathfrak{H}^{2} \rightarrow \mathcal{H}^{2}$ be a boundary relation with the Weyl family $M(\cdot) \in \widetilde{R}(\mathcal{H})$. Assume that $\Gamma_{0}\left(\widehat{\mathfrak{N}}_{\lambda}(T)\right)=\mathcal{H}_{0}$ holds for all $\lambda \in \mathbb{C} \backslash \mathbb{R}$. Then:

(i) $\gamma(\lambda)$ admits a single-valued closure for all $\lambda \in \mathbb{C} \backslash \mathbb{R}$;

(ii) $\mathcal{H}_{0} \subset \operatorname{dom} \gamma(\lambda)^{* *}=\operatorname{dom} \gamma(\mu)^{* *} \subset \operatorname{clos} \mathcal{H}_{0}, \quad \lambda, \mu \in \mathbb{C} \backslash \mathbb{R}$;

(iii) $\mathcal{H}_{0} \subset \operatorname{dom} \gamma(\lambda)^{*} \gamma(\mu), \lambda, \mu \in \mathbb{C} \backslash \mathbb{R}$;

(iv) $\gamma(\lambda) \in\left[\mathcal{H}_{0}, \mathfrak{N}_{\lambda}(T)\right]$ if and only if $\operatorname{Im} M_{s}(\lambda) \in\left[\mathcal{H}_{0}\right]$.

Proof. (i) This is a direct consequence of Proposition 4.9 .

(ii) It follows from (4.12) that there exists $c>0$, such that

$$
\frac{1}{c}\|\gamma(\mu)\|_{\mathfrak{H}} \leq\|\gamma(\lambda)\|_{\mathfrak{H}} \leq c\|\gamma(\mu)\|_{\mathfrak{H}},
$$

which shows that the topology induced on $\mathcal{H}_{0}$ by the form $(\gamma(\lambda) h, \gamma(\lambda) k)_{\mathfrak{H}}$ does not depend on $\lambda \in \mathbb{C} \backslash \mathbb{R}$. Therefore, the domain of the closure of this form is also independent of $\lambda \in \mathbb{C} \backslash \mathbb{R}$, and clearly the closure is given by $\left(\gamma(\lambda)^{* *} h, \gamma(\lambda)^{* *} k\right)_{\mathfrak{H}}$, $h, k \in \operatorname{dom} \gamma(\lambda)^{* *}$, where $\gamma(\lambda)^{* *}: \mathcal{H} \rightarrow \mathfrak{H}$ is the closure of $\gamma(\lambda), \lambda \in \mathbb{C} \backslash \mathbb{R}$. 
(iii) It follows from (4.8) that for all $h, k \in \mathcal{H}_{0}$

$$
(\lambda-\bar{\mu})(\gamma(\lambda) h, \gamma(\mu) k)_{\mathfrak{H}}=\left(h,\left(M_{s}(\lambda)^{*}-M_{s}(\mu)\right) k\right)_{\mathcal{H}} .
$$

Hence, for $k \in \operatorname{dom} \gamma(\mu)=\mathcal{H}_{0}$ and $\lambda \neq \bar{\mu}$,

$$
\begin{aligned}
\sup _{h \in \mathcal{H}_{0}} \frac{\left|(\gamma(\lambda) h, \gamma(\mu) k)_{\mathfrak{H}}\right|}{\|h\|} & =\sup _{h \in \mathcal{H}_{0}} \frac{\left|\left(h,\left(M_{s}(\lambda)^{*}-M_{s}(\mu)\right) k\right)_{\mathcal{H}}\right|}{|\lambda-\bar{\mu}|\|h\|} \\
& \leq \frac{\left\|\left(M_{s}(\lambda)^{*}-M_{s}(\mu)\right) k\right\|_{\mathcal{H}}}{|\lambda-\bar{\mu}|}<\infty,
\end{aligned}
$$

which means that $\gamma(\mu) k \in \operatorname{dom} \gamma(\lambda)^{*}$. Hence, $\mathcal{H}_{0} \subset \operatorname{dom} \gamma(\lambda)^{*} \gamma(\mu)$.

(iv) This follows immediately from the identity

$$
\begin{aligned}
\frac{\left(\operatorname{Im} M_{s}(\lambda) h, h\right)_{\mathfrak{H}}}{\operatorname{Im} \lambda} & =\frac{\left(M_{s}(\lambda) h, h\right)_{\mathcal{H}}-\left(M_{s}(\lambda)^{*} h, h\right)_{\mathcal{H}}}{\lambda-\bar{\lambda}} \\
& =(\gamma(\lambda) h, \gamma(\lambda) h)_{\mathfrak{H}},
\end{aligned}
$$

which is valid for all $h \in \mathcal{H}_{0}$.

Proposition 4.25. Assume that the Weyl family $M(\cdot)$ belongs to the subclass $\widetilde{R}_{\text {inv }}(\mathcal{H})$, so that (4.21) holds with a linear subspace $\mathcal{H}_{0} \subset \mathcal{H}$. Then the operator part of the Weyl family $M(\lambda)=M_{s}(\lambda) \oplus M_{\infty}$ has the operator representation where $\gamma(\mu)^{*}: \mathfrak{H} \rightarrow \operatorname{clos} \mathcal{H}_{0}$.

Proof. This follows immediately from the identity

$$
\frac{\left(M_{s}(\lambda) h, k\right)_{\mathcal{H}}-\left(M_{s}(\mu)^{*} h, k\right)_{\mathcal{H}}}{\lambda-\bar{\mu}}=(\gamma(\lambda) h, \gamma(\mu) k)_{\mathfrak{H}}, \quad h, k \in \mathcal{H}_{0},
$$

the identity (4.12), and part (iii) of Proposition 4.24 .

Under the assumption of domain invariance the symmetric relation $A_{0}=\operatorname{ker} \Gamma_{0}$ is essentially selfadjoint (cf. Corollary 4.12), so that its closure $A_{0}^{* *}=A_{0}^{*}$ is a selfadjoint extension of $S$. Recall that under these circumstances the identity (4.12) now holds for all $\lambda, \mu \in \mathbb{C} \backslash \mathbb{R}$ :

$$
\gamma(\lambda) h=\left[I+(\lambda-\mu)\left(A_{0}-\lambda\right)^{-1}\right] \gamma(\mu) h, \quad h \in \Gamma_{0}\left(\widehat{\mathfrak{N}}_{\lambda}(T)\right), \quad \lambda, \mu \in \mathbb{C} \backslash \mathbb{R} .
$$

Denote by $A_{s}$ the (orthogonal) operator part of $A_{0}^{* *}$, so that $A_{0}^{* *}$ admits the decomposition

$$
A_{0}^{* *}=A_{s} \oplus A_{\infty}, \quad A_{\infty}=\{0\} \times \operatorname{mul} A_{0}^{* *} .
$$

Let $P$ be the orthogonal projection onto $\mathfrak{H}_{s}=\overline{\operatorname{dom}} A_{0}^{* *}$, so that $I-P$ is the orthogonal projection onto mul $A_{0}^{* *}$. Let $E(t)$ be the spectral family (of orthogonal projections) of $A_{0}^{* *}$, so that in particular $\operatorname{ker} E(\infty)=\operatorname{ran}(I-P)$. Then the operator $A_{s}$ in $\mathfrak{H}_{s}$ satisfies

$$
I_{\mathfrak{H}_{s}}+(\lambda-\mu)\left(A_{s}-\lambda\right)^{-1}=\int_{\mathbb{R}} \frac{t-\mu}{t-\lambda} d E(t) .
$$

In view of (4.25) the identity (4.24) may now be rewritten as follows (with $\lambda, \mu \in$ $\mathbb{C} \backslash \mathbb{R}):$

(4.26) $\gamma(\lambda) h=(I-P) \gamma(\mu) h+\left[I_{\mathfrak{H}_{s}}+(\lambda-\mu)\left(A_{s}-\lambda\right)^{-1}\right] P \gamma(\mu) h, \quad h \in \Gamma_{0}\left(\widehat{\mathfrak{N}}_{\lambda}(T)\right)$, which is an orthogonal decomposition. 
The next result is an extension of the integral representation (2.29) from the subclass $R[\mathcal{H}]$ to the subclass $\widetilde{R}_{i n v}(\mathcal{H})$ of Nevanlinna families.

Proposition 4.26. Assume that the Weyl family $M(\cdot)$ belongs to the subclass $\widetilde{R}_{\text {inv }}(\mathcal{H})$, so that (4.21) holds with a linear subspace $\mathcal{H}_{0} \subset \mathcal{H}$. Then the operator part of the Weyl family $M(\lambda)=M_{s}(\lambda) \oplus M_{\infty}$ has the integral representation

$$
\left(M_{s}(\lambda) h, h\right)_{\mathcal{H}}=a_{h}+b_{h} \lambda+\int_{\mathbb{R}}\left(\frac{1}{t-\lambda}-\frac{t}{t^{2}+1}\right) d \sigma_{h}(t), \quad h \in \mathcal{H}_{0},
$$

where $a_{h}=\left(\operatorname{Re} M_{s}(i) h, h\right)_{\mathcal{H}}, d \sigma_{h}(t)=\left(t^{2}+1\right) d(E(t) P \gamma(i) h, P \gamma(i) h)_{\mathfrak{H}}$,

$$
b_{h}=\|(I-P) \gamma(i) h\|_{\mathfrak{H}}^{2},
$$

$P$ is the orthogonal projection onto $\overline{\operatorname{dom}} A_{0}^{* *}$, and $E(t)$ is the spectral family of $A_{0}^{* *}$.

Proof. It follows from (4.23), (4.25), and (4.26) that for all $h \in \mathcal{H}_{0}=\operatorname{dom} M(\lambda)$ and $\mu=i$,

$$
\begin{aligned}
\left(M_{s}(\lambda) h, h\right)_{\mathcal{H}}= & \left(M_{s}(i)^{*} h, h\right)_{\mathcal{H}}+(\lambda+i)\|(I-P) \gamma(i) h\|_{\mathfrak{H}}^{2} \\
& +(\lambda+i)\left(\left[I+(\lambda-i)\left(A_{s}-\lambda\right)^{-1}\right] P \gamma(i) h, \gamma(i) h\right)_{\mathfrak{H}} \\
= & \left(M_{s}(i)^{*} h, h\right)_{\mathcal{H}}+(\lambda+i)\left(b_{h}+\int_{\mathbb{R}} \frac{t-i}{t-\lambda} d(E(t) P \gamma(i) h, P \gamma(i) h)_{\mathfrak{H}}\right) .
\end{aligned}
$$

Since

$$
\operatorname{Im}\left(M_{s}(i) h, h\right)_{\mathcal{H}}=b_{h}+\int_{\mathbb{R}} d(E(t) P \gamma(i) h, P \gamma(i) h)_{\mathfrak{H}}=b_{h}+\int_{\mathbb{R}} \frac{d \sigma_{h}(t)}{t^{2}+1},
$$

one obtains (4.27).

The coefficient (4.28) of the linear term in the integral representation (4.27) may be obtained by a limiting procedure.

Proposition 4.27. Assume that the Weyl family $M(\cdot)$ belongs to the subclass $\widetilde{R}_{\text {inv }}(\mathcal{H})$, so that (4.21) holds with a linear subspace $\mathcal{H}_{0} \subset \mathcal{H}$. Then for all $\mu \in \mathbb{C} \backslash \mathbb{R}$ and $h \in \mathcal{H}_{0}$,

$$
\lim _{y \rightarrow \infty} \frac{\left(M_{s}(i y) h, h\right)_{\mathcal{H}}}{i y}=\|(I-P) \gamma(i) h\|_{\mathfrak{H}}^{2}=\|(I-P) \gamma(\mu) h\|_{\mathfrak{H}}^{2} .
$$

In particular, with $h \in \mathcal{H}_{0}$,

$$
\lim _{y \rightarrow \infty} \frac{\left(M_{s}(i y) h, h\right)_{\mathcal{H}}}{i y}=0 \quad \text { if and only if } \gamma(\mu) h \in \overline{\operatorname{dom}} A_{0}^{* *} .
$$

Proof. The first equality in (4.29) is implied by (4.27) and (4.28). To obtain the second equality in (4.29) observe that as a consequence of (4.26) the following limiting result holds:

$$
\lim _{y \rightarrow \infty}(\gamma(i y) h, \gamma(\mu) h)_{\mathfrak{H}}=\|(I-P) \gamma(\mu) h\|_{\mathfrak{H}}^{2} .
$$

Now the statement is implied by (4.23). The statement (4.30) is an immediate consequence of (4.29).

Corollary 4.28. Assume that the Weyl family $M(\cdot)$ belongs to the subclass $\widetilde{R}_{\text {inv }}(\mathcal{H})$ and let $S$ be an operator, i.e., mul $S=\{0\}$. Then $A_{0}^{* *}$ is an operator if and only if

$$
\lim _{y \rightarrow \infty} \frac{\left(M_{s}(i y) h, h\right)_{\mathcal{H}}}{i y}=0 \quad \text { for every } h \in \mathcal{H}_{0} .
$$


Proof. Let $A_{0}^{* *}$ be an operator. Then it follows from Proposition 4.27 that (4.31) holds.

Conversely, assume that (4.31) holds. If $S$ is simple, Proposition 4.27 implies that $\mathfrak{N}_{\lambda}(T), \lambda \in \mathbb{C} \backslash \mathbb{R}$, are orthogonal to mul $A_{0}^{* *}$. Since

$$
\mathfrak{H}=\overline{\operatorname{span}}\left\{\mathfrak{N}_{\lambda}(T): \lambda \in \mathbb{C} \backslash \mathbb{R}\right\},
$$

this implies that mul $A_{0}^{* *}=\{0\}$. In the general case, when $S$ is not necessarily simple, decompose the operator $S$ as an orthogonal sum $S_{0} \oplus S_{1}$ with a simple symmetric operator $S_{0}$ and a selfadjoint operator $S_{1}$. Then the result follows from the fact that $M(\cdot)$ is the Weyl family of the simple part $S_{1}$ of $S$.

As a direct consequence of (4.25) and (4.26) one obtains

$$
\|\gamma(\lambda) h\|_{\mathfrak{H}}^{2}=\|(I-P) \gamma(\mu) h\|_{\mathfrak{H}}^{2}+\int_{\mathbb{R}}\left|\frac{t-\mu}{t-\lambda}\right|^{2}\|d E(t) P \gamma(\mu) h\|_{\mathfrak{H}}^{2} .
$$

It also follows from (4.26) that if $\gamma(\lambda) h \in \operatorname{dom} A_{0}^{* *}$ for some $\lambda \in \mathbb{C} \backslash \mathbb{R}$, then the same is true for all $\lambda \in \mathbb{C} \backslash \mathbb{R}$.

Proposition 4.29. Assume that the Weyl family $M(\cdot)$ belongs to the subclass $\widetilde{R}_{\text {inv }}(\mathcal{H})$ and let $h \in \mathcal{H}_{0}=\Gamma_{0}\left(\widehat{\mathfrak{N}}_{\lambda}(T)\right), \lambda \in \mathbb{C} \backslash \mathbb{R}$. Then

$$
\sup _{y>0} y\left(\operatorname{Im} M_{s}(i y) h, h\right)_{\mathcal{H}}<\infty \quad \Leftrightarrow \quad \gamma(\mu) h \in \operatorname{dom} A_{0}^{* *}, \quad \mu \in \mathbb{C} \backslash \mathbb{R} .
$$

Proof. It follows from (4.8) that

$$
y\left(\frac{M_{s}(i y)-M_{s}(i y)^{*}}{2 i} h, h\right)_{\mathcal{H}}=y^{2}\|\gamma(i y) h\|_{\mathfrak{H}}^{2} .
$$

Combining (4.34) with (4.32) leads to

$$
y \operatorname{Im}\left(M_{s}(i y) h, h\right)_{\mathcal{H}}=y^{2}\|(I-P) \gamma(i) h\|_{\mathfrak{H}}^{2}+y^{2} \int_{\mathbb{R}} \frac{t^{2}+1}{t^{2}+y^{2}} d\left\|E_{t} P \gamma(i) h\right\|_{\mathfrak{H}}^{2} .
$$

An application of Lebesgue's monotone convergence theorem gives

$$
\sup _{y>0} y^{2} \int_{\mathbb{R}} \frac{t^{2}+1}{t^{2}+y^{2}} d\left\|E_{t} P \gamma(i) h\right\|_{\mathfrak{H}}^{2}=\int_{\mathbb{R}}\left(t^{2}+1\right) d\left\|E_{t} P \gamma(i) h\right\|_{\mathfrak{H}}^{2} .
$$

Now (4.35) and (4.36) show that the left-hand side of (4.35) is uniformly bounded for $y>0$ if and only if $(I-P) \gamma(i) h=0$ and $P \gamma(i) h \in \operatorname{dom} A_{0}^{* *}$.

Define the subclass $\widetilde{R}_{\text {inv }}^{s}(\mathcal{H})$ as the set of all Nevanlinna families $M(\cdot) \in \widetilde{R}_{\text {inv }}(\mathcal{H})$ for which $\operatorname{ker}\left(\operatorname{Im} M_{s}(\lambda)\right)=\{0\}$ for all $\lambda \in \mathbb{C} \backslash \mathbb{R}$.

Corollary 4.30. Let the Weyl family $M(\cdot)$ belong to the subclass $\widetilde{R}_{\text {inv }}^{s}(\mathcal{H})$. Moreover, assume that $A_{0}$ is selfadjoint and mul $S=\{0\}$. Then mul $T=\{0\}$ if and only if for every $h \in \mathcal{H} \backslash\{0\}$

$$
\lim _{y \rightarrow \infty} \frac{\left(M_{s}(i y) h, h\right)_{\mathcal{H}}}{i y}=0 \quad \text { and } \quad \lim _{y \rightarrow \infty} y\left(\operatorname{Im} M_{s}(i y) h, h\right)_{\mathcal{H}}=\infty .
$$

Proof. If (4.37) holds, then $A_{0}$ is an operator and $\mathfrak{N}_{\lambda}(T) \cap \operatorname{dom} A_{0}=\{0\}, \lambda \in \mathbb{C} \backslash \mathbb{R}$. Now Proposition 4.20 shows that $\operatorname{mul} T=\{0\}$.

Conversely, assume that mul $T=\{0\}$. Then by Proposition $4.20 \mathfrak{N}_{\lambda}(T) \cap$ $\operatorname{dom} A_{0}=\{0\}$ for every $\lambda \in \mathbb{C} \backslash \mathbb{R}$, and this implies (4.37), since $\gamma(\lambda)$ is injective (see (4.22) $)$. 
Remark 4.31. The results in this subsection are generalizations of similar statements which are well known for $Q$-functions of symmetric operators and Weyl functions of ordinary boundary triplets, i.e., for the subclass $R^{u}[\mathcal{H}]$ of Nevanlinna functions; see e.g. [26], 28].

4.5. Forbidden lineals. The concept of a forbidden (isometric) operator $V$ in the framework of von Neumann's theory was originally introduced by M.A. Krasnosel'skil in [23. The connection between the operator $V$ and limit values of the characteristic function was discovered by A.V. Straus [37. In the case of ordinary boundary triplets the so-called forbidden lineal has been introduced and studied in [28]; cf. also [16].

Definition 4.32. Let $\Gamma: \mathfrak{H}^{2} \rightarrow \mathcal{H}^{2}$ be a boundary linear relation and let $T=$ $\operatorname{dom} \Gamma$. The forbidden lineal of $\Gamma$ is defined by

$$
\mathcal{F}_{\Gamma}=\Gamma(\{0\} \times \operatorname{mul} T) .
$$

In this subsection the forbidden lineal of $\Gamma$ will be characterized by using the asymptotic properties of the Weyl family $M(\cdot)$, under the assumptions that $A_{0}$ is selfadjoint, and the operator part $M_{s}(\cdot)$ of $M(\cdot)$ has a bounded imaginary part for $\lambda \in \mathbb{C} \backslash \mathbb{R}$. The approach given below is rather straightforward, and the proof of the main statement in Proposition 4.34 is essentially simpler than the one used earlier in the case of ordinary boundary triplets.

Proposition 4.33. Let $A_{0}=A_{0}^{*}$ and let $P$ be the orthogonal projection onto $\overline{\operatorname{dom}} A_{0}$. Let $h \in \Gamma_{0}\{0,-\omega\}$ for some $\omega \in \operatorname{mul} T$ and let $\operatorname{Im} M_{s}(\lambda), \lambda \in \mathbb{C} \backslash \mathbb{R}$, be bounded. Then:

(i) $\gamma(\lambda) h \rightarrow 0$ strongly in $\mathcal{H}$ as $\lambda=i y \rightarrow \infty$;

(ii) $\lambda \gamma(\lambda) h \rightarrow-P \omega$ strongly in $\mathfrak{H}$ as $\lambda=i y \rightarrow \infty$;

(iii) the following strong limit exists:

$$
M_{s}(i \infty) h:=\lim _{y \rightarrow \infty} M_{s}(i y) h=M_{s}(\bar{\mu}) h-\gamma(\mu)^{*} P \omega .
$$

Proof. It follows from Proposition 4.20 that $h \in \operatorname{dom} \gamma(\lambda)$ and

$$
\gamma(\lambda) h=\left(A_{0}-\lambda\right)^{-1} \omega, \quad \lambda \in \mathbb{C} \backslash \mathbb{R} .
$$

The statements (i) and (ii) are immediate from the representation (4.39). Moreover, (iii) is implied by (4.23), since $\gamma(\mu)^{*}$ is bounded due to Proposition 4.24.

Proposition 4.34. Let $\Gamma: \mathfrak{H}^{2} \rightarrow \mathcal{H}^{2}$ be a boundary linear relation such that $A_{0}=A_{0}^{*}$ and $\operatorname{Im} M_{s}(\lambda)$ is a bounded operator for $\lambda \in \mathbb{C} \backslash \mathbb{R}$. Then

$$
\operatorname{dom} \mathcal{F}_{\Gamma}=\left\{h \in \mathcal{H}: \sup _{y>0} y\left(\operatorname{Im} M_{s}(i y) h, h\right)<\infty\right\},
$$

and the forbidden lineal $\mathcal{F}_{\Gamma}$ admits the representation

$$
\mathcal{F}_{\Gamma}=\left\{\left\{h, M_{s}(i \infty) h\right\}: h \in \operatorname{dom} \mathcal{F}_{\Gamma}\right\} \widehat{+} \Gamma\left(\{0\} \times \operatorname{mul} A_{0}\right) .
$$

Proof. The following characterization concerning $\operatorname{dom} \mathcal{F}_{\Gamma}$ is implied by Proposition 4.20

$$
h \in \operatorname{dom} \mathcal{F}_{\Gamma} \text { if and only if } \gamma(\lambda) h \in \operatorname{dom} A_{0} .
$$

Combining (4.42) with Proposition 4.29 gives the description of $\operatorname{dom} \mathcal{F}$ in (4.40). 
Next assume that $\left\{h, h^{\prime}\right\} \in \Gamma\{0,-\omega\}$, where $\omega \in \operatorname{mul} T$. Then

$$
\left\{\left(\begin{array}{l}
0 \\
h
\end{array}\right),\left(\begin{array}{l}
-\omega \\
-h^{\prime}
\end{array}\right)\right\} \in \widetilde{A}
$$

Also consider the elements

$$
\left\{\left(\begin{array}{c}
\gamma(\lambda) h \\
h
\end{array}\right),\left(\begin{array}{c}
\lambda \gamma(\lambda) h \\
-M_{s}(\lambda) h
\end{array}\right)\right\} \in \widetilde{A}, \quad \lambda \in \mathbb{C} \backslash \mathbb{R}
$$

It follows from Proposition 4.33 that with $\lambda=i y \rightarrow \infty$ these elements converge to

$$
\left\{\left(\begin{array}{l}
0 \\
h
\end{array}\right),\left(\begin{array}{c}
-P \omega \\
-M_{s}(i \infty) h
\end{array}\right)\right\} \in \widetilde{A}
$$

Now subtract from this element the element in (4.43), so that

$$
\left\{\left(\begin{array}{l}
0 \\
h
\end{array}\right),\left(\begin{array}{c}
-P \omega \\
-M_{s}(i \infty) h
\end{array}\right)\right\}-\left\{\left(\begin{array}{l}
0 \\
h
\end{array}\right),\left(\begin{array}{c}
-\omega \\
-h^{\prime}
\end{array}\right)\right\}=\left\{\left(\begin{array}{l}
0 \\
0
\end{array}\right),\left(\begin{array}{c}
(I-P) \omega \\
h^{\prime}-M_{s}(i \infty) h
\end{array}\right)\right\} \in \widetilde{A} .
$$

This shows that $\left\{0, h^{\prime}-M_{s}(i \infty) h\right\} \in \Gamma\left(\{0\} \times\right.$ mul $\left.A_{0}\right)$. Hence,

$$
\left\{h, h^{\prime}\right\}=\left\{h, M_{s}(i \infty) h\right\} \widehat{+}\left\{0, h^{\prime}-M_{s}(i \infty) h\right\},
$$

proving one inclusion in (4.41). The reverse inclusion is obvious from (4.40) and (4.45).

Corollary 4.35. Let $\Gamma: \mathfrak{H}^{2} \rightarrow \mathcal{H}^{2}$ be a boundary relation, which is single-valued and satisfies the assumptions of Proposition 4.34. Then

$$
\operatorname{mul} \mathcal{F}_{\Gamma}=\Gamma\left(\{0\} \times \operatorname{mul} A_{0}\right) .
$$

\section{Special Boundary RElations AND their Weyl families}

5.1. Ordinary boundary triplets. A combination of Definition 1.1 of an ordinary boundary triplet for the case of a densely defined symmetric operator from [20] (see also [15, 28]) and the adaptation for the case of a nondensely defined symmetric operator leads to the following definition. Note that now the adjoint $S^{*}$ of a symmetric operator $S$ in $\mathfrak{H}$ is a closed linear relation in $\mathfrak{H}$; it can be considered as a Hilbert space with the graph norm.

Definition $5.1(20])$. Let $S$ be a closed symmetric operator in a Hilbert space $\mathfrak{H}$ with equal defect numbers. A triplet $\Pi=\left\{\mathcal{H}, \Gamma_{0}, \Gamma_{1}\right\}$, where $\mathcal{H}$ is a Hilbert space with $\operatorname{dim} \mathcal{H}=n_{ \pm}(S)$ and $\Gamma_{i} \in\left[S^{*}, \mathcal{H}\right]$, is said to be an ordinary boundary triplet for $S^{*}$, if:

(A1) the abstract Green's identity (1.7) holds;

(A2) the mapping $\Gamma:=\left\{\Gamma_{0}, \Gamma_{1}\right\}: S^{*} \rightarrow \mathcal{H}^{2}$ is surjective.

Lemma 5.2. Let $\Gamma:\left(\mathfrak{H}^{2}, J_{\mathfrak{H}}\right) \rightarrow\left(\mathcal{H}^{2}, J_{\mathcal{H}}\right)$ be isometric and let $T=\operatorname{dom} \Gamma$ satisfy $S \subset T \subset S^{*}$. If $T$ is dense in $S^{*}$ and $\operatorname{ran} \Gamma=\mathcal{H}^{2}$, then $S=\operatorname{ker} \Gamma$ has equal defect numbers, $T=S^{*}$, and $\Gamma$ is a bounded single-valued unitary relation.

Proof. By assumptions $(\operatorname{dom} \Gamma)^{[\perp]}=T^{*}=S \subset \operatorname{dom} \Gamma$. Hence, Corollary 2.6] shows that $\Gamma$ is a unitary single-valued relation which is bounded with $T=\operatorname{dom} \Gamma=S^{*}$. In particular, $\operatorname{ker} \Gamma=T^{*}=S$. Since mul $\Gamma=\{0\}$, the defect numbers of $S$ are equal by Lemma 2.14 . 
Proposition 5.3. A triplet $\left\{\mathcal{H}, \Gamma_{0}, \Gamma_{1}\right\}$ is an ordinary boundary triplet for $S^{*}$ if and only if $\Gamma=\left\{\Gamma_{0}, \Gamma_{1}\right\}: \mathfrak{H}^{2} \mapsto \mathcal{H}^{2}$ is a boundary relation for $S^{*}$ such that

$$
\operatorname{ran} \Gamma=\mathcal{H}^{2} .
$$

Proof. Let $\left\{\mathcal{H}, \Gamma_{0}, \Gamma_{1}\right\}$ be an ordinary boundary triplet for $S^{*}$. Note that (A1) and (A2) mean that $\Gamma: \mathfrak{H}^{2} \mapsto \mathcal{H}^{2}$ is an isometric operator from the Kreŭn space $\left(\mathfrak{H}^{2}, J_{\mathfrak{H}}\right)$ into the Kreun space $\left(\mathcal{H}^{2}, J_{\mathcal{H}}\right)$ with $\operatorname{dom} \Gamma=S^{*}$ and $\operatorname{ran} \Gamma=\mathcal{H}^{2}$. By Lemma $5.2 \Gamma$ is unitary and (G1) and (G2) are satisfied.

Conversely, if $\Gamma: \mathfrak{H}^{2} \rightarrow \mathcal{H}^{2}$ is a boundary relation for $S^{*}$ with $\operatorname{ran} \Gamma=\mathcal{H}^{2}$, then $\Gamma$ is unitary and $\operatorname{dom} \Gamma=S^{*}$. The assumptions in Definition 5.1 are now obtained from Lemma 5.2

The kernels $A_{i}:=\operatorname{ker} \Gamma_{i}, i=0,1$, define two selfadjoint extensions of $S$. Associated with $\Pi$ are two functions which are holomorphic on $\rho\left(A_{0}\right)$.

Definition 5.4 ([15, 16]). Let $\Pi=\left\{\mathcal{H}, \Gamma_{0}, \Gamma_{1}\right\}$ be an ordinary boundary triplet for $S^{*}$. Then the $\gamma$-field $\gamma(\cdot)$ and the Weyl function $M(\cdot)$ corresponding to $\Pi$ are defined by

$$
\widehat{\gamma}(\lambda):=\left(\Gamma_{0} \uparrow \widehat{\mathfrak{N}}_{\lambda}\right)^{-1}, \quad \gamma(\lambda):=\pi_{1}\left(\Gamma_{0}\left\lceil\widehat{\mathfrak{N}}_{\lambda}\right)^{-1}, \quad M(\lambda)=\Gamma_{1} \widehat{\gamma}(\lambda),\right.
$$

with $\lambda \in \rho\left(A_{0}\right)$. Here $\widehat{\mathfrak{N}}_{\lambda}:=\widehat{\mathfrak{N}}_{\lambda}\left(S^{*}\right)$ and $\pi_{1}$ stands for the projection onto the first component of $\mathcal{H} \oplus \mathcal{H}$.

In this case the corresponding Weyl function $M(\cdot)$ belongs to the class $R^{u}[\mathcal{H}]$. In fact, $M(\cdot)$ determines the pair $\left\{S, A_{0}\right\}$ up to unitary equivalence (cf. [26]), and, conversely, for every such function $M(\cdot) \in R^{u}[\mathcal{H}]$ there exist a symmetric operator $S$ and an ordinary boundary triplet for $S^{*}$ whose Weyl function is equal to $M(\cdot)$.

It was shown in [15], 28, that $\gamma(\cdot)$ and $M(\cdot)$ satisfy (4.12) and the following identity:

$$
M(\lambda)-M(\mu)^{*}=(\lambda-\bar{\mu}) \gamma(\mu)^{*} \gamma(\lambda), \quad \lambda, \mu \in \rho\left(A_{0}\right) .
$$

This means that the $\gamma$-field $\gamma(\cdot)$ of $S$ in Definition 5.4 is generated by $A_{0}$ and that $M(\cdot)$ is a $Q$-function of the pair $\left\{S, A_{0}\right\}$.

5.2. Generalized boundary triplets. A more general definition of a boundary triplet for a symmetric operator $S$ with equal defect numbers appears in [16]. This motivates the next lemma.

Lemma 5.5. Let $\Gamma$ be an isometric relation from $\left(\mathfrak{H}^{2}, J_{\mathfrak{H}}\right)$ to $\left(\mathcal{H}^{2}, J_{\mathcal{H}}\right)$ with the properties

(i) $\operatorname{ran} \Gamma_{0}=\mathcal{H}$;

(ii) $A_{0}:=\operatorname{ker} \Gamma_{0}$ is essentially selfadjoint in $\mathfrak{H}$,

where $\Gamma_{0}$ is as defined in (3.6). Then $\Gamma$ is a unitary relation from $\left(\mathfrak{H}^{2}, J_{\mathfrak{H}}\right)$ to $\left(\mathcal{H}^{2}, J_{\mathcal{H}}\right)$.

Proof. Let $\{\widehat{k}, \widehat{g}\} \in \Gamma^{[*]}$ with $\widehat{k}=\left\{k, k^{\prime}\right\}$ and $\widehat{g}=\left\{g, g^{\prime}\right\}$. By assumption (i) $\left\{\widehat{s},\left\{k, t^{\prime}\right\}\right\} \in \Gamma$ for some $\widehat{s}=\left\{s, s^{\prime}\right\} \in \mathfrak{H}^{2}$ and $t^{\prime} \in \mathcal{H}$. Since $\Gamma^{-1} \subset \Gamma^{[*]}$, one concludes that

$$
\left\{\left\{0, k^{\prime}-t^{\prime}\right\}, \widehat{g}-\widehat{s}\right\}=\{\widehat{k}, \widehat{g}\}-\left\{\left\{k, t^{\prime}\right\}, \widehat{s}\right\} \in \Gamma^{[*]} .
$$


Moreover, the assumption (i) and Lemma 4.10 imply that $A_{0}$ is closed. Thus, by the assumption (ii) $A_{0}$ is a selfadjoint relation in $\mathfrak{H}$. The condition $\widehat{f} \in A_{0}$ means that $\left\{\widehat{f},\left\{0, h^{\prime}\right\}\right\} \in \Gamma$ for some $h^{\prime} \in \mathcal{H}$. Now it follows from (5.4) that for all $\widehat{f} \in A_{0}$,

$$
\left(J_{\mathfrak{H}} \widehat{f}, \widehat{g}-\widehat{s}\right)_{\mathfrak{H}^{2}}=\left(J_{\mathcal{H}}\left\{0, h^{\prime}\right\},\left\{0, k^{\prime}-t^{\prime}\right\}\right)_{\mathcal{H}^{2}}=0 .
$$

Therefore, $\widehat{g}-\widehat{s} \in A_{0}^{*}=A_{0}$ by assumption (ii). Hence, $\left\{\widehat{g}-\widehat{s},\left\{0, v^{\prime}\right\}\right\} \in \Gamma$ for some $v^{\prime} \in \mathcal{H}$, and (5.4) implies that $\left\{\left\{0, k^{\prime}-t^{\prime}-v^{\prime}\right\},\{0,0\}\right\} \in \Gamma^{[*]}$. This means that for all $\{\widehat{f}, \widehat{h}\} \in \Gamma$,

$$
0=\left(J_{\mathfrak{H}} \widehat{f},\{0,0\}\right)_{\mathfrak{H}^{2}}=\left(J_{\mathcal{H}} \widehat{h},\left\{0, k^{\prime}-t^{\prime}-v^{\prime}\right\}\right)_{\mathcal{H}^{2}}=i\left(h, k^{\prime}-t^{\prime}-v^{\prime}\right)_{\mathcal{H}},
$$

and now (i) yields $k^{\prime}-t^{\prime}-v^{\prime}=0$. Hence $\{\widehat{g}, \widehat{k}\}=\left\{\widehat{g}-\widehat{s},\left\{0, v^{\prime}\right\}\right\}+\left\{\widehat{s},\left\{k, t^{\prime}\right\}\right\} \in \Gamma$, and therefore $\Gamma^{[*]} \subset \Gamma^{-1}$.

Now recall the definition of a generalized boundary triplet as given in 16].

Definition 5.6 (16]). Let $S$ be a closed symmetric operator in a Hilbert space $\mathfrak{H}$ with equal defect numbers and let $T$ be a linear relation in $\mathfrak{H}$ such that $S \subset$ $T \subset \operatorname{clos} T=S^{*}$. Then the triplet $\left\{\mathcal{H}, \Gamma_{0}, \Gamma_{1}\right\}$, where $\mathcal{H}$ is a Hilbert space and $\Gamma=\left\{\Gamma_{0}, \Gamma_{1}\right\}$ is a single-valued linear mapping from $T$ to $\mathcal{H}^{2}$, is said to be a generalized boundary triplet for $S^{*}$, if:

(S1) the abstract Green's identity (1.7) holds for all $\widehat{f}=\left\{f, f^{\prime}\right\}, \widehat{g}=\left\{g, g^{\prime}\right\} \in T$;

(S2) $\operatorname{ran} \Gamma_{0}=\mathcal{H}$;

(S3) $A_{0}:=\operatorname{ker} \Gamma_{0}$ is a selfadjoint linear relation in $\mathfrak{H}$.

By definition $A_{0} \subset \operatorname{dom} \Gamma=T$, so that $A_{0}$ is a selfadjoint extension of $S$.

Proposition 5.7. A triplet $\left\{\mathcal{H}, \Gamma_{0}, \Gamma_{1}\right\}$ is a generalized boundary triplet for $S^{*}$ if and only if $\Gamma=\left\{\Gamma_{0}, \Gamma_{1}\right\}: \mathfrak{H}^{2} \mapsto \mathcal{H}^{2}$ is a boundary relation for $S^{*}$ such that

$$
\operatorname{mul} \Gamma=\{0\}, \quad \Gamma_{0}\left(\widehat{\mathfrak{N}}_{\lambda}(T)\right)=\mathcal{H}, \quad \lambda \in \mathbb{C} \backslash \mathbb{R} .
$$

In this case the corresponding Weyl family $M(\lambda)$ belongs to the class $R^{s}[\mathcal{H}]$. Conversely, every $R^{s}[\mathcal{H}]$-function is the Weyl function of some generalized boundary triplet $\left\{\mathcal{H}, \Gamma_{0}, \Gamma_{1}\right\}$.

Proof. Let $\left\{\mathcal{H}, \Gamma_{0}, \Gamma_{1}\right\}$ be a generalized boundary triplet for $S^{*}$. Then $\Gamma=\left\{\Gamma_{0}, \Gamma_{1}\right\}$ is unitary by Lemma 5.5, and hence it is a boundary relation for $S^{*}$ with mul $\Gamma=$ $\{0\}$. It follows from the assumption (S3) that $S^{*}=A_{0} \widehat{+} \widehat{\mathfrak{N}}_{\lambda}\left(S^{*}\right), \lambda \in \mathbb{C} \backslash \mathbb{R}$, and since $A_{0} \subset T$, the equality

$$
T=A_{0} \widehat{+} \widehat{\mathfrak{N}}_{\lambda}(T), \quad \lambda \in \mathbb{C} \backslash \mathbb{R},
$$

also holds. This together with (S2) gives

$$
\Gamma_{0}\left(\widehat{\mathfrak{N}}_{\lambda}(T)\right)=\Gamma_{0}(T)=\mathcal{H}, \quad \lambda \in \mathbb{C} \backslash \mathbb{R} .
$$

The statement $M(\lambda) \in R^{s}[\mathcal{H}]$ is obtained from Proposition 4.7.

Conversely, let $\Gamma$ be a boundary relation for $S^{*}$ with the properties (5.5). Then $\mathcal{H}=\Gamma_{0}\left(\widehat{\mathfrak{N}}_{\lambda}(T)\right) \subset \operatorname{ran} \Gamma_{0}$, so that $\operatorname{ran} \Gamma_{0}=\mathcal{H}$, i.e., (S2) is satisfied. Also the property (S3) is obtained from $\Gamma_{0}\left(\mathfrak{N}_{\lambda}(T)\right)=\mathcal{H}$ by using Proposition 4.15; cf. Corollary 4.17. The remaining conditions for generalized boundary triplets are clearly satisfied.

It follows from Theorem 3.9 and Propositions 4.5, 4.7 that every $R^{s}[\mathcal{H}]$-function is the Weyl function of some generalized boundary triplet. 
The converse part of Proposition [5.7 was first proved in [16]. The next result collects some further properties of generalized boundary triplets.

Proposition 5.8 (16]). Let $\left\{\mathcal{H}, \Gamma_{0}, \Gamma_{1}\right\}$ be a generalized boundary triplet for $S^{*}$. Then:

(i) $T=A_{0} \widehat{+} \widehat{\mathfrak{N}}_{\lambda}(T)$ for every $\lambda \in \mathbb{C} \backslash \mathbb{R}$;

(ii) $\operatorname{clos} \Gamma_{1}\left(A_{0}\right)=\mathcal{H}$ and $\overline{\operatorname{ran}} \Gamma=\mathcal{H}^{2}$;

(iii) the restriction $\Gamma_{0}: \widehat{\mathfrak{N}}_{\lambda}(T) \rightarrow \mathcal{H}$ is a closed mapping for every $\lambda \in \mathbb{C} \backslash \mathbb{R}$;

(iv) the equalities (5.2) define an $\left[\mathcal{H}, \widehat{\mathfrak{N}}_{\lambda}\right]$-valued function $\widehat{\gamma}(\cdot)$, an $\left[\mathcal{H}, \mathfrak{N}_{\lambda}\right]$-valued function $\gamma(\cdot)$, and an $[\mathcal{H}]$-valued function $M(\cdot)$, which are holomorphic on $\mathbb{C} \backslash \mathbb{R}$ and satisfy the identities (4.12) and (5.3).

Proof. (i) This was shown in the proof of Proposition 5.7, cf. (5.6).

(ii) By Proposition $2.3 \operatorname{ran} \Gamma$ is dense in $\mathcal{H}^{2}$. Now assume that $h \perp \Gamma_{1}\left(A_{0}\right)$. Then $\{k, h\} \perp \Gamma\left(A_{0}\right)$ in $\mathcal{H}^{2}$ for every $k \in \mathcal{H}$. Since $\left\{-M(\lambda)^{*} h, h\right\} \perp \Gamma\left(\widehat{\mathfrak{N}}_{\lambda}\right)$ in $\mathcal{H}^{2}$, it follows from (i) that $\left\{-M(\lambda)^{*} h, h\right\} \perp \operatorname{ran} \Gamma$ and, therefore, $h=0$.

(iii) By Proposition 4.9 the mapping $\Gamma_{0}: \widehat{\mathfrak{N}}_{\lambda}(T) \rightarrow \mathcal{H}, \lambda \in \mathbb{C} \backslash \mathbb{R}$, is closable. Moreover, it is closed since $\Gamma_{0}\left(\widehat{\mathfrak{N}}_{\lambda}(T)\right)=\mathcal{H}$.

(iv) The operators $\widehat{\gamma}(\lambda), \lambda \in \mathbb{C} \backslash \mathbb{R}$, are bounded by the closed graph theorem and thus also the operators $\gamma(\lambda), \lambda \in \mathbb{C} \backslash \mathbb{R}$, are bounded. The corresponding statement for $M(\lambda)$ was shown in Proposition [5.9. The remaining statements are immediate from Proposition 4.11 and the operator representation (4.23).

5.3. Boundary relations with Weyl functions in $R[\mathcal{H}]$. In this subsection the class of boundary relations whose Weyl functions belong to the class $R[\mathcal{H}]$ is considered.

Proposition 5.9. Let $\mathcal{H}$ and $\mathfrak{H}$ be Hilbert spaces and let $\Gamma: \mathfrak{H}^{2} \rightarrow \mathcal{H}^{2}$ be a (possibly multi-valued) linear relation such that:

(B1) Green's identity (3.1) holds;

(B2) $\operatorname{ran} \Gamma_{0}=\mathcal{H}$;

(B3) $A_{0}:=\operatorname{ker} \Gamma_{0}$ is a selfadjoint linear relation in $\mathfrak{H}$.

Then $\Gamma: \mathfrak{H}^{2} \rightarrow \mathcal{H}^{2}$ is a boundary relation for $S^{*}:=(\operatorname{ker} \Gamma)^{*}$ such that

$$
\Gamma_{0}\left(\widehat{\mathfrak{N}}_{\lambda}(T)\right)=\mathcal{H}, \quad \lambda \in \mathbb{C} \backslash \mathbb{R} .
$$

Conversely, every boundary relation $\Gamma: \mathfrak{H}^{2} \rightarrow \mathcal{H}^{2}$ satisfying (5.7) also satisfies the conditions (B1)-(B3). In this case the corresponding Weyl function belongs to the class $R[\mathcal{H}]$, and moreover, every $R[\mathcal{H}]$-function is the Weyl function of some boundary relation $\Gamma: \mathfrak{H}^{2} \rightarrow \mathcal{H}^{2}$ with the properties (B1)-(B3).

Proof. The proof is completely analogous to that of Proposition 5.7 and will be omitted.

Proposition 5.10. Let $\Gamma: \mathfrak{H}^{2} \rightarrow \mathcal{H}^{2}$ be a boundary relation satisfying the conditions (B1)-(B3). Then:

(i) $T=A_{0} \widehat{+} \widehat{\mathfrak{N}}_{\lambda}(T)$ for every $\lambda \in \mathbb{C} \backslash \mathbb{R}$;

(ii) $\left(\Gamma_{0}\left\lceil\widehat{\mathfrak{N}}_{\lambda}(T)\right)^{-1}: \mathcal{H} \rightarrow \widehat{\mathfrak{N}}_{\lambda}(T)\right.$ is closed bounded and single-valued for every $\lambda \in \mathbb{C} \backslash \mathbb{R}$

(iii) the equalities (5.2) define $\left[\mathcal{H}, \widehat{\mathfrak{N}}_{\lambda}\right]$-valued, $\left[\mathcal{H}, \mathfrak{N}_{\lambda}\right]$-valued, and $[\mathcal{H}]$-valued functions $\widehat{\gamma}(\cdot), \gamma(\cdot)$, and $M(\cdot)$, respectively, which are holomorphic on $\mathbb{C} \backslash \mathbb{R}$ and satisfy the identities (4.12) and (5.3). 
Proof. The proof is analogous to that of Proposition 5.8. The main difference is that the mapping $\left.\Gamma_{0} \uparrow \widehat{\mathfrak{N}}_{\lambda}(T)\right): \widehat{\mathfrak{N}}_{\lambda}(T) \rightarrow \mathcal{H}$ may be multi-valued, but it still has a trivial kernel and its inverse is a closed bounded single-valued mapping for every $\lambda \in \mathbb{C} \backslash \mathbb{R}$ by Proposition 4.9. The identity (5.3) is implied by (4.23).

\section{EXAmples}

In this section a number of illustrative examples are presented. Each example by itself shows some characteristic behaviour of boundary relations.

Example 6.1. Let $A$ be a selfadjoint relation in a Hilbert space $\mathfrak{H}$ and let $B$ be a selfadjoint relation in a Hilbert space $\mathcal{H}$. Define the linear relation $\widetilde{A}$ in $\mathfrak{H} \oplus \mathcal{H}$ by $\widetilde{A}=A \oplus(-B)$, i.e.,

$$
\widetilde{A}=\left\{\left\{\left(\begin{array}{l}
f \\
h
\end{array}\right),\left(\begin{array}{c}
f^{\prime} \\
-h^{\prime}
\end{array}\right)\right\}:\left\{f, f^{\prime}\right\} \in A,\left\{h, h^{\prime}\right\} \in B\right\} .
$$

It is clear that $\widetilde{A}$ is selfadjoint. The transform

$$
\Gamma=\mathcal{J}^{-1}(\widetilde{A})=\left\{\left\{\left(\begin{array}{c}
f \\
f^{\prime}
\end{array}\right),\left(\begin{array}{c}
h \\
h^{\prime}
\end{array}\right)\right\}:\left\{f, f^{\prime}\right\} \in A,\left\{h, h^{\prime}\right\} \in B\right\}
$$

defines a boundary relation $\Gamma: \mathfrak{H}^{2} \rightarrow \mathcal{H}^{2}$ for $S^{*}=A$ with

$$
T=\operatorname{dom} \Gamma=A, \quad S=\operatorname{ker} \Gamma=A, \quad \operatorname{ran} \Gamma=B, \quad \operatorname{mul} \Gamma=B .
$$

In particular, this implies that $A_{0}=A_{1}=S=S^{*}$ are selfadjoint. The corresponding Weyl family $M(\lambda)=\Gamma\left(\widehat{\mathfrak{N}}_{\lambda}(T)\right)$ is given by $M(\lambda) \equiv B$. Since $\operatorname{ran} \Gamma_{0}=\operatorname{dom} B$, $M(\lambda) \in \widetilde{R}[\mathcal{H}]$ if and only if $\operatorname{dom} B$ is closed (in which case $B$ is the orthogonal sum of a bounded selfadjoint operator and a closed multi-valued part). Furthermore, $\mathfrak{N}_{\lambda}\left(S^{*}\right)=\mathfrak{N}_{\lambda}(A)=\{0,0\}$, so that the $\gamma$-field satisfies $\gamma(\lambda) h=0$ for all $h \in \operatorname{dom} B$ and hence its closure is single-valued.

Example 6.1 gives a realization for a constant Nevanlinna family $M(\lambda) \equiv B$ as a Weyl family of a boundary relation $\Gamma$. Observe that the symmetry property $M(\lambda)^{*}=M(\bar{\lambda})$ forces that $B=B^{*}$. In Example 6.1 the boundary relation $\Gamma$ is not minimal. The following result expresses this situation more explicitly.

Corollary 6.2. Let $\Gamma: \mathfrak{H}^{2} \rightarrow \mathcal{H}^{2}$ be an arbitrary boundary relation whose Weyl function satisfies $M(\lambda)=B, \lambda \in \mathbb{C} \backslash \mathbb{R}$. If $\Gamma$ is minimal, then $\mathfrak{H}=\{0\}$, and in particular $S=T=S^{*}$.

Proof. Consider $\left\{\widehat{f_{\lambda}}, \widehat{h}\right\} \in \Gamma$ with $\lambda \in \mathbb{C} \backslash \mathbb{R}$. The identity (3.4) with $\lambda=\mu$ shows that

$$
(\lambda-\bar{\lambda})\left\|f_{\lambda}\right\|^{2}=\left(h^{\prime}, h\right)-\left(h, h^{\prime}\right)=0,
$$

since $B$ is selfadjoint. This implies that $\widehat{f}_{\lambda}=\{0,0\}$. Now the assumption that $\Gamma: \mathfrak{H}^{2} \rightarrow \mathcal{H}^{2}$ is minimal yields $\mathfrak{H}=\overline{\operatorname{span}}\left\{\mathfrak{N}_{\lambda}(T): \lambda \in \mathbb{C} \backslash \mathbb{R}\right\}=\{0\}$. In this case $S=T=S^{*}=\{0,0\}$.

In Proposition 3.7 a boundary relation for an arbitrary closed symmetric relation $S$ in a Hilbert space $\mathfrak{H}$ is constructed. In the next example some further properties of the corresponding boundary relation are considered. 
Example 6.3. Let $\widetilde{A}$ be the linear relation in $\mathfrak{H} \oplus \mathfrak{H}$ given by (3.10) and let $\Gamma=\mathcal{J}^{-1}(\widetilde{A})$ be the corresponding boundary relation for $S^{*}$. Then $\Gamma$ satisfies the following equalities:

$$
\operatorname{ker} \Gamma=\operatorname{mul} \Gamma=S, \quad \operatorname{dom} \Gamma=\operatorname{ran} \Gamma=S^{*} .
$$

Moreover, the corresponding Weyl family $M(\lambda)$ is given by

$$
M(\lambda)=S \widehat{+} \widehat{\mathfrak{N}}_{\lambda}\left(S^{*}\right), \quad \lambda \in \mathbb{C} \backslash \mathbb{R} .
$$

To see this assume $\left\{f_{2}, f_{2}^{\prime}\right\} \in M(\lambda)$. Then it follows from (3.5) that

$$
\left\{\left\{f_{\lambda}, \lambda f_{\lambda}\right\},\left\{f_{2}, f_{2}^{\prime}\right\}\right\} \in \Gamma \quad \text { for some } \quad\left\{f_{\lambda}, \lambda f_{\lambda}\right\} \in S^{*} .
$$

By (3.10) this shows $P_{\mathfrak{N}}\left(\left\{f_{2}, f_{2}^{\prime}\right\}-\left\{f_{\lambda}, \lambda f_{\lambda}\right\}\right)=0$, which implies that

$$
\left\{f_{2}, f_{2}^{\prime}\right\}-\left\{f_{\lambda}, \lambda f_{\lambda}\right\} \in S
$$

so that $\left\{f_{2}, f_{2}^{\prime}\right\} \in S \widehat{+} \widehat{\mathfrak{N}}_{\lambda}\left(S^{*}\right)$. Hence $M(\lambda) \subset S \widehat{+} \widehat{\mathfrak{N}}_{\lambda}\left(S^{*}\right)$, and the reverse inclusion can be seen immediately. Furthermore,

$$
\operatorname{mul} M(\lambda)=\operatorname{mul} S, \quad \lambda \in \mathbb{C} \backslash \mathbb{R} .
$$

To see this, consider an arbitrary element in $M(\lambda)$ with $\lambda \in \mathbb{C} \backslash \mathbb{R}$ :

$$
\left\{h, h^{\prime}\right\} \hat{+}\left\{f_{\lambda}, \lambda f_{\lambda}\right\} \in M(\lambda) \quad \text { with } \quad\left\{h, h^{\prime}\right\} \in S, \quad\left\{f_{\lambda}, \lambda f_{\lambda}\right\} \in \widehat{\mathfrak{N}}_{\lambda}\left(S^{*}\right),
$$

so that $\left(h^{\prime}, f_{\lambda}\right)=\bar{\lambda}\left(h, f_{\lambda}\right)$. If $h+f_{\lambda}=0$, then $\left\{-f_{\lambda}, h^{\prime}\right\} \in S$ and $\left(h^{\prime}, f_{\lambda}\right) \in \mathbb{R}$. Hence $f_{\lambda}=0$, and consequently, $h=0$. Thus mul $M(\lambda) \subset$ mul $S$ and the reverse inclusion is obvious.

In this example $M(\cdot) \in \widetilde{R}(\mathfrak{H})$. According to Proposition 3.5 the boundary relation $\Gamma$ is minimal if and only if the selfadjoint relation $\widetilde{A}=\mathcal{J}(\Gamma)$ in $\mathfrak{H} \oplus \mathfrak{H}$ is minimal with respect to $\mathfrak{H}$, which is equivalent to $S$ being simple. Hence, if $S$ is simple, in which case $S$ is an operator, this model provides a minimal realization for the Weyl family $M(\lambda)$ in 6.1). Moreover, its multi-valued part is trivial due to (6.2). Therefore, if $S$ is simple, $M(\cdot) \in R(\mathfrak{H})$.

Note that $\operatorname{ran} \Gamma_{0}=\operatorname{dom} S^{*}$ and $\operatorname{ran} \Gamma_{1}=\operatorname{ran} S^{*}$. Moreover,

$$
\begin{aligned}
& A_{0}=\operatorname{ker} \Gamma_{0}=\left\{\left\{f_{1}, f_{1}^{\prime}\right\} \in S^{*}: P_{\mathfrak{N}} \widehat{f}_{1}=P_{\mathfrak{N}}\left\{0, f_{2}^{\prime}\right\},\left\{0, f_{2}^{\prime}\right\} \in S^{*}\right\}, \\
& A_{1}=\operatorname{ker} \Gamma_{1}=\left\{\left\{f_{1}, f_{1}^{\prime}\right\} \in S^{*}: P_{\mathfrak{N}} \widehat{f}_{1}=P_{\mathfrak{N}}\left\{f_{2}, 0\right\},\left\{f_{2}, 0\right\} \in S^{*}\right\} .
\end{aligned}
$$

This implies, in a similar way as above, that

$$
A_{0}=S \widehat{+}\left\{0, \operatorname{mul} S^{*}\right\}, \quad A_{1}=S \widehat{+}\left\{\operatorname{ker} S^{*}, 0\right\} .
$$

The extension $A_{0}$ of $S$ is symmetric and, in general, not selfadjoint. The defect numbers of $A_{0}$ are called semi-defect numbers of $S$; see 23 .

Remark 6.4. If in Example $6.3, S$ is a densely defined symmetric operator, then (6.3) shows that $A_{0}=\operatorname{ker} \Gamma_{0}$ coincides with $S$. Therefore, $A_{0}$ need not be selfadjoint and, in general, it can have arbitrary (equal or unequal) defect numbers.

The next example gives a boundary relation whose Weyl function belongs to $R_{\text {inv }}(\mathcal{H}) \backslash R[\mathcal{H}]$.

Example 6.5. Let $B$ be a closed densely defined linear operator in $\mathfrak{H}$ and let $\widetilde{A}$ be the operator determined by the block form

$$
\widetilde{A}=\left(\begin{array}{cc}
0 & B \\
B^{*} & 0
\end{array}\right) .
$$


By definition $\widetilde{A}$ is a selfadjoint operator in $\mathfrak{H} \oplus \mathfrak{H}$ whose graph is given by

$$
\widetilde{A}=\left\{\left\{\left(\begin{array}{c}
f \\
h
\end{array}\right),\left(\begin{array}{c}
B h \\
B^{*} f
\end{array}\right)\right\}: h \in \operatorname{dom} B, f \in \operatorname{dom} B^{*}\right\} .
$$

The transform

$$
\Gamma=\mathcal{J}^{-1}(\widetilde{A})=\left\{\left\{\left(\begin{array}{c}
f \\
B h
\end{array}\right),\left(\begin{array}{c}
h \\
-B^{*} f
\end{array}\right)\right\}: h \in \operatorname{dom} B, f \in \operatorname{dom} B^{*}\right\}
$$

defines a boundary relation for $S^{*}=\mathfrak{H} \oplus \overline{\text { ran }} B$ in $\mathfrak{H} \oplus \mathfrak{H}$ with

$$
\begin{gathered}
T=\operatorname{dom} \Gamma=\operatorname{dom} B^{*} \times \operatorname{ran} B, \quad S=\operatorname{ker} \Gamma=\operatorname{ker} B^{*} \times\{0\}, \\
\operatorname{ran} \Gamma=\operatorname{dom} B \times \operatorname{ran} B^{*}, \quad \operatorname{mul} \Gamma=\operatorname{ker} B \times\{0\} .
\end{gathered}
$$

Hence, $\Gamma$ is single-valued if and only if ker $B=\{0\}$ and, since mul $\Gamma \cap\{0, \mathfrak{H}\}=\{0\}$, the corresponding Weyl family $M(\lambda)$ is operator-valued. Clearly, $\{f, \lambda f\} \in T$ if and only if

Hence,

$$
f=\frac{1}{\lambda} B h, \quad h \in \operatorname{dom} B^{*} B
$$

$$
\Gamma_{0}\left(\widehat{\mathfrak{N}}_{\lambda}(T)\right)=\operatorname{dom} B^{*} B, \quad \Gamma_{1}\left(\widehat{\mathfrak{N}}_{\lambda}(T)\right)=\operatorname{ran} B^{*} B,
$$

and the corresponding $\gamma$-field and the Weyl family are given by

$$
\gamma(\lambda)=\frac{1}{\lambda} B, \quad M(\lambda)=-\frac{1}{\lambda} B^{*} B
$$

on $\operatorname{dom} B^{*} B$. In particular, $\Gamma_{0}\left(\widehat{\mathfrak{N}}_{\lambda}(T)\right)$ and $\Gamma_{1}\left(\widehat{\mathfrak{N}}_{\lambda}(T)\right)$ are independent of $\lambda \in$ $\mathbb{C} \backslash \mathbb{R}$. In general they do not coincide with $\operatorname{ran} \Gamma_{0}$ and $\operatorname{ran} \Gamma_{1}$, since

$$
\operatorname{ran} \Gamma_{0}=\operatorname{dom} B, \quad \operatorname{ran} \Gamma_{1}=\operatorname{ran} B^{*} .
$$

In this example $A_{0}$ and $A_{1}$ are essentially selfadjoint in $\mathfrak{H}$, since

$$
A_{0}=\operatorname{ker} \Gamma_{0}=\operatorname{dom} B^{*} \times\{0\}, \quad A_{1}=\operatorname{ker} \Gamma_{1}=\operatorname{ker} B^{*} \times \operatorname{ran} B .
$$

Furthermore, the equality $M(\lambda)-M(\mu)^{*}=(\lambda-\bar{\mu}) \gamma(\mu)^{*} \gamma(\lambda)$ is satisfied.

The next example gives a boundary relation whose Weyl function $M(\cdot)$ typically appears in the case of bounded perturbations of a selfadjoint operator. Observe that in this example $-M(\cdot)^{-1}$ need not belong to the class $\widetilde{R}_{\text {inv }}(\mathcal{H})$.

Example 6.6. Let $B$ be bounded and let $D$ and $E$ be selfadjoint operators in $\mathfrak{H}$.

Define $\widetilde{A}$ by the block form

$$
\widetilde{A}=\left(\begin{array}{cc}
D & B \\
B^{*} & -E
\end{array}\right)
$$

Then $\widetilde{A}$ is a selfadjoint operator in $\mathfrak{H} \oplus \mathfrak{H}$ whose graph is given by

$$
\widetilde{A}=\left\{\left\{\left(\begin{array}{l}
f \\
h
\end{array}\right),\left(\begin{array}{c}
D f+B h \\
B^{*} f-E h
\end{array}\right)\right\}: f \in \operatorname{dom} D, h \in \operatorname{dom} E\right\} .
$$

The transform

$$
\Gamma=\mathcal{J}^{-1}(\widetilde{A})=\left\{\left\{\left(\begin{array}{c}
f \\
D f+B h
\end{array}\right),\left(\begin{array}{c}
h \\
E h-B^{*} f
\end{array}\right)\right\}: f \in \operatorname{dom} D, h \in \operatorname{dom} E\right\}
$$

defines a boundary relation in $\mathfrak{H} \oplus \mathfrak{H}$ with

$$
\begin{aligned}
& T=\operatorname{dom} \Gamma=D \hat{+}(\{0\} \times B(\operatorname{dom} E)), \quad S=\operatorname{ker} \Gamma=D \uparrow \operatorname{ker} B^{*}, \\
& \operatorname{ran} \Gamma=E \widehat{+}\left(\{0\} \times B^{*}(\operatorname{dom} D)\right), \quad \operatorname{mul} \Gamma=E \uparrow \operatorname{ker} B,
\end{aligned}
$$


so that $S^{*}=\operatorname{clos}(D \widehat{+}(\{0\} \times \overline{\operatorname{ran}} B))$. Since mul $\Gamma \cap\{0, \mathfrak{H}\}=\{0\}$, the corresponding Weyl family $M(\lambda)$ is operator-valued. Moreover,

$$
\operatorname{ran} \Gamma_{0}=\operatorname{dom} E, \quad \operatorname{ran} \Gamma_{1}=B^{*}(\operatorname{dom} D)+\operatorname{ran} E .
$$

Observe, that $\{f, \lambda f\} \in T$ if and only if

$$
f=-(D-\lambda)^{-1} B h .
$$

Hence,

$$
\Gamma_{0}\left(\widehat{\mathfrak{N}}_{\lambda}(T)\right)=\operatorname{dom} E, \quad \Gamma_{1}\left(\widehat{\mathfrak{N}}_{\lambda}(T)\right)=\operatorname{ran}\left(B^{*}(D-\lambda)^{-1} B+E\right),
$$

and the corresponding $\gamma$-field and the Weyl family are given by

$$
\gamma(\lambda)=-(D-\lambda)^{-1} B, \quad M(\lambda)=B^{*}(D-\lambda)^{-1} B+E,
$$

on $\operatorname{dom} M(\lambda)$. In particular, $\Gamma_{0}\left(\widehat{\mathfrak{N}}_{\lambda}(T)\right)=\operatorname{ran} \Gamma_{0}$ for every $\lambda \in \mathbb{C} \backslash \mathbb{R}$, while $\Gamma_{1}\left(\widehat{\mathfrak{N}}_{\lambda}(T)\right)$ in general depends on $\lambda \in \mathbb{C} \backslash \mathbb{R}$ and need not coincide with $\operatorname{ran} \Gamma_{1}$. The formulas

$$
\begin{aligned}
& A_{0}=\operatorname{ker} \Gamma_{0}=D, \\
& A_{1}=\operatorname{ker} \Gamma_{1}=\left\{\{f, D f+B h\}: f \in \operatorname{dom} D, h \in \operatorname{dom} E, B^{*} f=E h\right\},
\end{aligned}
$$

show that $A_{0}$ is selfadjoint, while $A_{1}$ need not be essentially selfadjoint in $\mathfrak{H}$.

However, if for instance $E=0$, then $A_{1}$ takes the form

$$
A_{1}=\left\{\left\{f, f^{\prime}\right\} \in T: B^{*} f=0\right\}=S \widehat{+}(\{0\} \times \operatorname{ran} B) .
$$

By Theorem 4.13 the equality $\Gamma_{1}\left(\widehat{\mathfrak{N}}_{\lambda}(T)\right)=\operatorname{ran} \Gamma_{1}$ holds for every $\lambda \in \mathbb{C} \backslash \mathbb{R}$ if and only if $A_{1}$ is selfadjoint: this holds if, for instance, $E=0$ and $\operatorname{ran} B$ is finitedimensional, in which case $A_{1}$ is in fact the generalized Friedrichs extension of $S$. Since $A_{0}$ is a selfadjoint extension of $S, S$ has equal defect numbers. Observe that $A_{0}=S$ if and only if $B=0$, so that when $B \neq 0, S$ is not selfadjoint.

On the other hand, $A_{1}=S$ if and only if the following implication holds:

$$
B^{*} f=E h, \quad f \in \operatorname{dom} D \Rightarrow h=0 \text { and } B^{*} f=0,
$$

or equivalently,

$$
B^{*}(\operatorname{dom} D) \cap \operatorname{ran} E=\{0\} \quad \text { and } \quad \operatorname{ker} E=0 .
$$

In this case $A_{1}$ cannot be essentially selfadjoint (unless $B=0$ ) and thus $\Gamma_{1}\left(\widehat{\mathfrak{N}}_{\lambda}(T)\right.$ ) must depend on $\lambda \in \mathbb{C} \backslash \mathbb{R}$. Observe that there exist bounded selfadjoint operators $B=B^{*}$ and $E=E^{*}$ with the properties

$$
\operatorname{ran} B \cap \operatorname{ran} E=\{0\}, \quad \operatorname{ker} E=\operatorname{ker} B=\{0\},
$$

so that also the conditions in (6.4) can be satisfied.

In the next example the Weyl function takes a constant value in the lower halfplane which is a symmetric operator with defect numbers $(1,0)$. In fact, this example can be seen as a special case of Example 6.3. It involves some first-order differential operators on the halflines.

Example 6.7. Let $S_{+}$and $S_{-}$be the minimal differential operator generated in $\mathfrak{H}=L_{2}(0, \infty)$ and $\mathcal{H}=L_{2}(-\infty, 0)$, respectively, by the expression $i D$. Let $\widetilde{A}$ be the selfadjoint operator in $\mathfrak{H} \oplus \mathcal{H}$ given by

$$
\left\{\left\{\left(\begin{array}{l}
y_{+} \\
y_{-}
\end{array}\right),\left(\begin{array}{l}
-i y_{+}^{\prime} \\
-i y_{-}^{\prime}
\end{array}\right)\right\}: y_{+} \in W_{2}^{1}(0, \infty), y_{-} \in W_{2}^{1}(-\infty, 0), y_{+}(0+)=y_{-}(0-)\right\} \text {. }
$$


Clearly, the operator $\widetilde{A}$ is minimal with respect to the space $\mathcal{H}$ in the sense of (2.23) since the symmetric operator $S_{+}$is simple; see Lemma 2.14. Then the linear relation $\Gamma=\mathcal{J}^{-1}(\widetilde{A}): \mathfrak{H}^{2} \rightarrow \mathcal{H}^{2}$ is a minimal boundary relation with

$$
\operatorname{dom} \Gamma=-S_{+}^{*}, \quad \operatorname{ker} \Gamma=-S_{+}, \quad \operatorname{ran} \Gamma=S_{-}^{*}, \quad \operatorname{mul} \Gamma=S_{-} .
$$

The symmetric extensions $A_{0}=\operatorname{ker} \Gamma_{0}$ and $A_{1}=\operatorname{ker} \Gamma_{1}$ are equal and coincide with $S=\operatorname{ker} \Gamma=-S_{+}$, which is a maximal symmetric operator. Furthermore, observe that

$$
\operatorname{ran} \Gamma_{0}=\operatorname{dom} S_{-}^{*}, \quad \operatorname{ran} \Gamma_{1}=\operatorname{ran} S_{-}^{*} .
$$

The defect subspaces $\mathfrak{N}_{\lambda}\left(S^{*}\right)$ are given by

$$
\mathfrak{N}_{\lambda}\left(S^{*}\right)=\operatorname{span}\left\{e^{i \lambda x}\right\}, \quad \lambda \in \mathbb{C}_{+}, \quad \mathfrak{N}_{\lambda}\left(S^{*}\right)=\{0\}, \quad \lambda \in \mathbb{C}_{-},
$$

and the corresponding Weyl family $M(\lambda)=\Gamma\left(\widehat{\mathfrak{N}}_{\lambda}\left(S^{*}\right)\right)$ has the form

$$
M(\lambda)=S_{-}^{*}, \quad \lambda \in \mathbb{C}_{+}, \quad M(\lambda)=S_{-}, \quad \lambda \in \mathbb{C}_{-} .
$$

Clearly, $M(\lambda)$ belongs to the class $R(\mathcal{H})$ and has the domain invariance property in each halfplane, but not on $\mathbb{C} \backslash \mathbb{R}$; cf. Proposition 4.11. In fact, each halfplane gives different behaviour. For $\lambda \in \mathbb{C}_{+}$the corresponding $\gamma$-field is given by

$$
\gamma(\lambda) h=h(0) e^{i \lambda x}, \quad h \in \operatorname{dom} S_{-}^{*},
$$

so that

$$
\|\gamma(\lambda) h\|^{2}=\frac{|h(0)|^{2}}{2 \operatorname{Im} \lambda}=\frac{\left(S_{-}^{*} h, h\right)-\left(h, S_{-}^{*} h\right)}{\lambda-\bar{\lambda}}, \quad h \in \operatorname{dom} S_{-}^{*} .
$$

Clearly (4.10) is not satisfied for $\lambda \in \mathbb{C}_{+}$, since $S_{-}^{*} \not \subset S_{-}$. In fact, the closure of the $\gamma$-field $\gamma(\lambda)$ is not single-valued for $\lambda \in \mathbb{C}_{+}$. To see this, define a sequence of smooth functions $h_{n} \in \mathcal{H}$ such that $h_{n}(0)=1$ and $h_{n} \rightarrow 0$ in $\mathcal{H}=L^{2}(-\infty, 0)$, in which case $\gamma(\lambda) h_{n}=e^{i \lambda x} \neq 0$. For $\lambda \in \mathbb{C}_{-}$the corresponding $\gamma$-field is given by

$$
\gamma(\lambda) h=0, \quad h \in \operatorname{dom} S_{-},
$$

and

$$
\|\gamma(\lambda) h\|^{2}=\frac{\left(S_{-} h, h\right)-\left(h, S_{-} h\right)}{\lambda-\bar{\lambda}}, \quad h \in \operatorname{dom} S_{-} .
$$

Clearly (4.10) is satisfied for $\lambda \in \mathbb{C}_{-}$, since $S_{-} \subset S_{-}^{*}$. Therefore, the closure of $\gamma(\lambda)$ is single-valued for $\lambda \in \mathbb{C}_{-}$and, in fact, $\gamma(\lambda) h=0$ for all $h \in \mathcal{H}$.

The last example demonstrates the applicability of boundary relations (as defined in the present paper) for treating boundary value problems involving partial differential operators.

Example 6.8. Let $A=A^{*}(\geq I)$ be a semibounded operator in a Hilbert space $\mathcal{H}$ and let $S$ be a minimal symmetric operator in $\mathfrak{H}=L_{2}((0, \infty), \mathcal{H})$ associated with the differential expression

$$
l[y]=-y^{\prime \prime}+A^{2} y .
$$

Define the operator $T$ as a restriction of $S^{*}$ to the domain

$$
\operatorname{dom} T=\left\{y \in \operatorname{dom} S^{*}: y(0), y^{\prime}(0) \in \mathcal{H}\right\}
$$

and the mapping $\Gamma: \operatorname{dom} T \rightarrow \mathcal{H}^{2}$ by

$$
\Gamma y=\left(\begin{array}{c}
\Gamma_{0} y \\
\Gamma_{1} y
\end{array}\right)=\left(\begin{array}{c}
y(0) \\
y^{\prime}(0)
\end{array}\right), \quad y \in \operatorname{dom} T .
$$


It will be proved that $\Gamma$ is a boundary relation for $S^{*}$ in the sense as defined in the present paper. Simple calculations show that

$$
(T y, z)-(y, T z)=\left(\Gamma_{1} y, \Gamma_{0} z\right)_{\mathcal{H}}-\left(\Gamma_{0} y, \Gamma_{1} z\right)_{\mathcal{H}}, \quad y, z \in \operatorname{dom} T,
$$

that is, the condition (G1) of Definition 3.1 (the Green's identity) is satisfied. Next, observe that the defect subspace $\mathfrak{N}_{\lambda}(T)$ consists of the vectors

$$
y_{\lambda}:=e^{-\sqrt{A^{2}-\lambda} t} h, \quad h \in \operatorname{dom} A .
$$

Since $\Gamma_{0} y_{\lambda}=h$ and $\Gamma_{0} y_{\lambda}=-\sqrt{A^{2}-\lambda} h$, the $\gamma$-field and the Weyl function corresponding to $\left\{\mathcal{H}, \Gamma_{0}, \Gamma_{1}\right\}$ are given by

$$
\gamma(\lambda) h=y_{\lambda}, \quad M(\lambda) h=-\sqrt{A^{2}-\lambda} h, \quad h \in \operatorname{dom} A .
$$

Indeed, the operator $M(\lambda)$ is maximal dissipative for all $\lambda \in \mathbb{C}_{+}$due to $A=A^{*}$. To see that the linear relation $\Gamma$ is closed, let $y_{n} \in \operatorname{dom} T$ and $y_{n} \rightarrow y, T y_{n} \rightarrow z$ in $\mathfrak{H}$ and $y_{n}(0) \rightarrow h, y_{n}^{\prime}(0) \rightarrow g$ in $\mathcal{H}$ as $n \rightarrow \infty$. Then $y \in \operatorname{dom} S^{*}$, since $S^{*}$ is a closed operator. It is shown in [20] that $y(0) \in \mathcal{H}_{-1 / 2}(A)$ and $y^{\prime}(0) \in \mathcal{H}_{-3 / 2}(A)$, where $\mathcal{H}_{\alpha}(A)=\operatorname{dom} A^{\alpha}$ if $\alpha \geq 0$, and it is the completion of $\mathcal{H}$ with respect to the norm $\|h\|_{\alpha}=\left\|A^{\alpha} h\right\|$ if $\alpha<0$. Moreover, $\mathfrak{N}_{\lambda}\left(S^{*}\right)=\left\{e^{-\sqrt{A^{2}-\lambda} t} h: h \in \mathcal{H}_{-1 / 2}(A)\right\}$. Since $y_{n}(0)$ converges to $y(0)$ in $\mathcal{H}_{-1 / 2}(A)$ and $y_{n}^{\prime}(0)$ converges to $y^{\prime}(0)$ in $\mathcal{H}_{-3 / 2}(A)$, one concludes that $y(0)=h \in \mathcal{H}, y^{\prime}(0)=g \in \mathcal{H}$ and, therefore, $y \in \operatorname{dom} T$. Finally, the graph of $T$ is dense in $S^{*}$. This follows from von Neumann's formula, since the defect subspace $\mathfrak{N}_{\lambda}(T)=\left\{e^{-\sqrt{A^{2}-\lambda} t} h: h \in \operatorname{dom} A\right\}$ is dense in $\mathfrak{N}_{\lambda}\left(S^{*}\right)$. Therefore, by Proposition $3.6 \Gamma$ is a boundary relation for $S^{*}$ and $M(\lambda)$ is the corresponding Weyl function.

Clearly, $M \in R_{\text {inv }}(\mathcal{H}) \backslash R[\mathcal{H}]$, since the function $-\sqrt{s^{2}-\lambda}$ is unbounded on $[1, \infty)$ for every $\lambda \in \mathbb{C} \backslash[1, \infty)$ and $\operatorname{dom} M(\lambda)=\mathcal{H}_{1}(A)$ for all $\lambda \in \mathbb{C} \backslash \mathbb{R}$.

Note that the proof of the fact that $\Gamma$ is a boundary relation for $S^{*}$ is rather simple. At the same time a construction of an ordinary boundary triplet for $S^{*}$ is a nontrivial problem, since, in general, boundary values $y(0)$ and $y^{\prime}(0)$ of $y \in \operatorname{dom} S^{*}$ do not belong to $\mathcal{H}$. M.L. Gorbachuk has shown (cf. 20]) that an ordinary boundary triplet $\left\{\mathcal{H}, \widetilde{\Gamma}_{0}, \widetilde{\Gamma}_{1}\right\}$ for $S^{*}$ can be chosen in the form

$$
\widetilde{\Gamma}_{0} y=\widehat{A}^{-1 / 2} y(0), \quad \widetilde{\Gamma}_{1} y=A^{1 / 2}\left(y^{\prime}(0)+\widehat{A} y(0)\right),
$$

where $\widehat{A}$ denotes the continuation of the operator $A$ acting from $\mathcal{H}_{\alpha}(A)$ to $\mathcal{H}_{\alpha+1}(A)$. In this case the corresponding Weyl function is $M(\lambda)=\left(A-\sqrt{A^{2}-\lambda}\right) A$; see [15].

In a forthcoming paper the usefulness of the concept of Weyl functions with unbounded values is demonstrated for the spectral analysis of selfadjoint extensions in Example 6.8. In particular, starting with the representation (6.6) of the Weyl function, it will be shown that for any $A(\geq I)$ the Friedrichs extension $S_{F}$ corresponding to the Dirichlet boundary value problem for the operator (6.5) has a purely absolutely continuous spectrum.

\section{REFERENCES}

[1] N.I. Achieser and I.M. Glasmann, Theorie der linearen Operatoren im Hilbertraum, 8., erweiterte Auflage, Akademie Verlag, Berlin, 1981. MR0661612 (83i:47001c)

[2] R. Arens, "Operational calculus of linear relations", Pacific J. Math., 11 (1961), 9-23. MR0123188 (23:A517)

[3] T.Ya. Azizov and I.S. Iokhvidov, Linear operators in spaces with indefinite metric, John Wiley and Sons, New York, 1989. MR.1033489 (90j:47042) 
[4] J. Behrndt and P. Jonas, "Boundary value problems with local generalized Nevanlinna functions in the boundary condition", Integral Equations Operator Theory, to appear (Preprint 01-2004, Institute of Mathematics, Technische Universität Berlin, 2004).

[5] C. Bennewitz, "Symmetric relations on a Hilbert space", Lect. Notes Math., 280 (1972), 212-218. MR0415380 (54:3468)

[6] L. de Branges, "Some Hilbert spaces of entire functions", Trans. Amer. Math. Soc., 105 (1962), 43-83. MR0143016 (26:583)

[7] M.S. Brodskii, Triangular and Jordan representations of linear operators, Nauka, Moscow, 1968. MR0259648(41:4283)

[8] J. Brüning and V. Geyler, "Geometric scattering on compact Riemannian manifolds", Dokl. Akad. Nauk, 389 (2003), 310-313. MR2003683(2004g:58039)

[9] J. Brüning, V. Geyler, and K. Pankrashkin, "On diagonal singularities of the Green functions for Schrödinger operators", J. Math. Phys., 46, 113508 (2005) (16 pages). MR2186783

[10] E.A. Coddington, "Extension theory of formally normal and symmetric subspaces", Mem. Amer. Math. Soc., 134 (1973), 1-80. MR0477855 (57:17357)

[11] V.A. Derkach, S. Hassi, M.M. Malamud, and H.S.V. de Snoo, "Generalized resolvents of symmetric operators and admissibility", Reports of the Department of Mathematics, University of Helsinki, Preprint 252 (2000), 51 pp.

[12] V.A. Derkach, S. Hassi, M.M. Malamud, and H.S.V. de Snoo, "Generalized resolvents of symmetric operators and admissibility", Methods of Functional Analysis and Topology, 6, no.3 (2000), 24-55. MR.1903120(2003b:47042)

[13] V.A. Derkach, S. Hassi, M.M. Malamud, and H.S.V. de Snoo, "Boundary relations and Weyl families", Rus. Dokl. Acad. Sci., 399, no. 2 (2004), 151-156 (Russian). MR2125963 (2006c:47013)

[14] V.A. Derkach and M.M. Malamud, "On Weyl function and Hermitian operators with gaps", Doklady Acad. Nauk SSSR, 293, no. 5 (1987), 1041-1046. MR0890193 (88k:47032)

[15] V.A. Derkach and M.M. Malamud, "Generalized resolvents and the boundary value problems for hermitian operators with gaps", J. Funct. Anal., 95 (1991), 1-95. MR1087947|(93d:47046)

[16] V.A. Derkach and M.M. Malamud, "The extension theory of hermitian operators and the moment problem", J. Math. Sciences, 73 (1995), 141-242. MR1318517 (95m:47009)

[17] A. Dijksma and H.S.V. de Snoo, "Selfadjoint extentions of symmetric subspaces", Pacific J. Math., 54, no. 1 (1974), 71-99. MR0361889 (50:14331)

[18] W.F. Donoghue, Monotone matrix functions and analytic continuation, Springer-Verlag, Berlin, Heidelberg, New York, 1974. MR0486556 (58:6279)

[19] F. Gestezy, N.J. Kalton, K. Makarov, and E.R. Tsekanovskiı̌, "Some applications of operator-valued Herglotz functions", Operator theory, system theory and related topics (Beer-Sheva/Rehovot, 1997), Oper. Theory Adv. Appl., 123 (2001), 271-321. MR1821917 (2002f:47049)

[20] V. I. Gorbachuk and M. L. Gorbachuk, Boundary value problems for operator differential equations. Translated and revised from the 1984 Russian original. Mathematics and its Applications (Soviet Series), 48, Kluwer Academic Publishers Group, Dordrecht, 1991. MR1154792 (92m:34133)

[21] I.S. Kac and M.G. Kreĭn, " $R$-functions - analytic functions mapping the upper halfplane into itself", Supplement to the Russian edition of F.V. Atkinson, Discrete and continuous boundary problems, Mir, Moscow 1968 (Russian) (English translation: Amer. Math. Soc. Transl. Ser. 2, 103 (1974), 1-18). MR0243149(39:4473)

[22] T. Kato, Perturbation theory for linear operators, Springer-Verlag, Berlin, Heidelberg, New York, 1966. MR0203473(34:3324)

[23] M.A. Krasnosel'skiı̌, "On selfadjoint extensions of Hermitian operators", Ukrainian Mat. Zh., 1, no. 1 (1949), 21-38. MR0047924 (13:954b)

[24] M.G. Krĕn and H. Langer, "On defect subspaces and generalized resolvents of Hermitian operator in a Pontryagin space", Funkts. Anal. i Prilozhen, 5, no. 2 (1971), 59-71; ibid. 5, no. 3 (1971), 54-69 (Russian) (English translation: Funct. Anal. Appl., 5 (1971), 136-146; ibid. 5 (1971), 217-228). MR.0282238 (43:7951a)

[25] M.G. Krein and H. Langer, "Uber die $Q$-function eines $\pi$-hermitescher Operators in Raume $\Pi_{\kappa} "$, Acta. Sci. Math. (Szeged), 34 (1973), 191-230. MR0318958(47:7504) 
[26] H. Langer and B. Textorius, "On generalized resolvents and $Q$-functions of symmetric linear relations (subspaces) in Hilbert space", Pacific J. Math., 72 (1977), 135-165. MR0463964 (57:3902)

[27] M. Lesch and M.M. Malamud, "On the deficiency indices and self-adjointness of symmetric Hamiltonian systems", J. Differential Equations, 189 (2003), 556-615. MR1964480 (2004f:34128)

[28] M.M. Malamud, "On the formula of generalized resolvents of a nondensely defined Hermitian operator", Ukr. Mat. Zh., 44, no. 2, (1992), 1658-1688. MR.1215039 (95i:47046)

[29] M.M. Malamud and S.M. Malamud, "Spectral theory of operator measures in Hilbert space", St. Petersburg Math. Journal, 15, no. 3 (2003), 1-77. MR2052164 (2005i:47008)

[30] M.A. Naimark, "On spectral functions of a symmetric operator", Izv. Akad. Nauk SSSR, Ser. Matem., 7 (1943), 285-296. MR0010790 (6:71d)

[31] B.C. Orcutt, Canonical differential equations, Doctoral dissertation, University of Virginia, 1969.

[32] R.S. Phillips, "Dissipative operators and hyperbolic systems of partial differential equations", Trans. Amer. Math. Soc., 90 (1959), 192-254. MR0104919(21:3669)

[33] R.S. Phillips, "The extension of dual subspaces invariant under an algebra", Proc. International Symp. on Linear spaces, Israel, 1960, Academic Press, 366-398. MR0133686 (24:A3512)

[34] Yu.L. Shmul'jan, "Theory of linear relations, and spaces with indefinite metric", Funkcional. Anal. i Priložen, 10, no. 1 (1976), 67-72. (Russian) MR0405165 (53:8960)

[35] I.M. Spitkovski1̌, "On the construction of a unitary operator by its diagonal blocks", Matem. Issledovaniya, Kishinev, 8, no. 4 (1973), 187-193. MR0350505 (50:2997)

[36] B. Sz.-Nagy and C. Foias, Harmonic analysis of operators in Hilbert space, Budapest, 1967. MR0275191 (43:948)

[37] A.V. Straus, "Characteristic functions of linear operators", Izv. Akad. Nauk. SSSR, Ser. Mat., 24 (1960), 43-74. (Russian) MR0140950 (25:4363)

[38] A.V. Štraus, "On selfadjoint operators in the orthogonal sum of Hilbert spaces", Dokl. Akad. Nauk SSSR, 144 (1962), 512-515. MR.0136994 (25:454)

[39] E.C. Titchmarsh, Eigenfunction expansions associated with second-order differential equations, Part One, second edition, Oxford University Press, Oxford, 1962. MR0176151|(31:426)

[40] H.Weyl, "Über gewöhnliche Differentialgleichungen mit Singularitäten und die zugehörigen Entwicklungen willkürlicher Funktionen", Math. Ann., 68 (1910), 220-269. MR1511560

Department of Mathematics, Donetsk National University, Universitetskaya str. 24, 83055 Donetsk, UkRAine

E-mail address: derkach@univ.donetsk.ua

Department of Mathematics and Statistics, University of Vaasa, P.O. Box 700, 65101 VAASA, Finland

E-mail address: sha@uwasa.fi

Department of Mathematics, Donetsk National University, Universitetskaya str. 24, 83055 Donetsk, Ukraine

E-mail address: mmm@univ.donetsk.ua

Department of Mathematics and Computing Science, University of Groningen, P.O.

Box 800, 9700 AV Groningen, The Netherlands

E-mail address: desnoo@math.rug.nl 